\title{
Embodied Presence \\ Evidence for embodiment of effectors when pointing in virtual environments
}

\author{
by \\ Robin Langerak
}

A dissertation submitted to the Faculty of Graduate and Postdoctoral Affairs in partial fulfillment of the requirements for the degree of

\author{
Doctor of Philosophy \\ in \\ Psychology
}

Carleton University

Ottawa, Ontario

(C) 2019

Robin Langerak 
This work is dedicated in memory of Dr. Anthony Whitehead, whose kindness, curiosity, and diverse wealth of knowledge inspired me and countless other Carleton University students to follow their passions and never let the rules get in the way. 


\section{ACKNOWLEDGEMENTS}

To my advisor Chris Herdman, this work would not have been possible without you and the innovative research facility you built at VSIM. Thank you for always being willing to entertain an idea - even if it's a little out there - while also helping me learn to stick to the plot.

Thanks to my committee Jo-Anne Lefevre and Craig Leth-Steensen for the thoughtprovoking questions and the insightful suggestions that improved the quality of this work.

Etelle Bourassa, thank-you for all the times that I left your office feeling better than when I walked in. Cecilia Jorgenson and Chunyun Ma, I cannot imagine this journey without all the support, perspective, and laughter that was shared in our writing group. Thank you.

Mike Chan-Reynolds, I would not be where I am without you. You gave me a toolbox and inspired my curiosity. Thank-you for believing in me when I was starting, encouraging me when I was stuck, and reminding me of my strengths as I move forward.

Matt Brown, thank-you for your support and your sarcasm. Both were important. You helped me trust my training and kept me honest when I wanted to gloss over the details.

Kevin Grignon, thanks for always going to bat for me and reminding me to ask for what I need. Thanks also to Cale Vardy and Eric Mahlstedt for their support as I finished this thesis.

Finally, I owe much gratitude to my family. Ben, you know how to take me seriously when I need a confidant and how to send me into a fit of giggles like no one else can. Thanks for bringing the balance of extremes that reminds me to always be me.

Mom, thanks for encouraging in me a feisty determination that must have backfired on you more than once. You told me to become a doctor instead of marrying one. I have you to thank for the grit that got me here and the rebellious spirit that keeps me conquering. 


\begin{abstract}
In this thesis, I proposed and tested the Embodied Presence Model (EPM), a framework aimed at examining the role of mental representations of the environment and body's effectors in supporting presence in virtual reality. A fundamental assumption of the EPM is that presence is a direct result of interactions between one's environment and one's effectors (e.g., hands) thus requiring a mental representation of both. The EPM was therefore informed by current perspectives from perceptual, cognitive, and neuropsychology literature on how the relationship between the environment and the body. Three experiments were conducted, each with approximately 50 participants. In all three experiments a virtual pointing task was used wherein participants made speeded pointing movements to virtual targets presented on the table in front of them the virtual environment. Behavioral data included latency measures (i.e., hand liftoff time and hand travel time) and landing accuracy of pointing movements. Subjective data were also collected using an adaptation of the Rubber Hand Illusion questionnaire. Experiment 1 served as a paradigm check and examined the kinematic characteristics of pointing movements made by the left and right hands. Experiment 2 examined the influence of changes to the virtual environments on pointing behaviour by introducing fire into the virtual environment. Experiment 3 examined the combined effects of altering the virtual environment - once again with the use of virtual fire - and the virtual effector by comparing pointing behaviour in a virtual hand and a virtual cube condition. Findings suggest that representations of the virtual environment and effectors both influence pointing behaviour, and that these factors may support embodied presence independently. A better understanding of these psychological underpinnings of embodied presence will improve the use of VR in a multitude of situations, including research, training and clinical applications.
\end{abstract}




\section{TABLE OF CONTENTS}

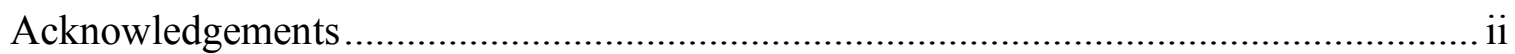

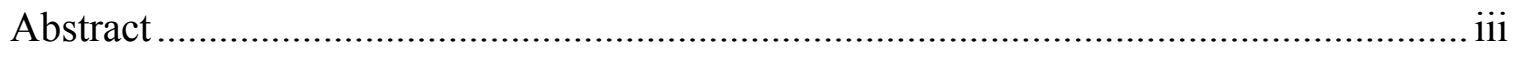

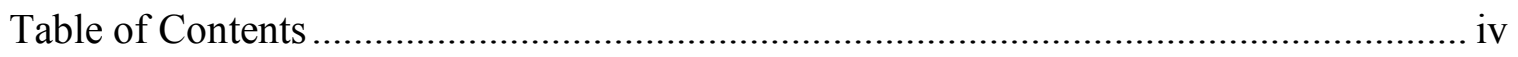

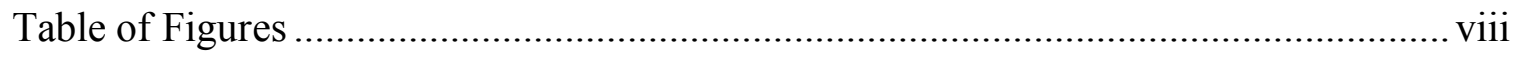

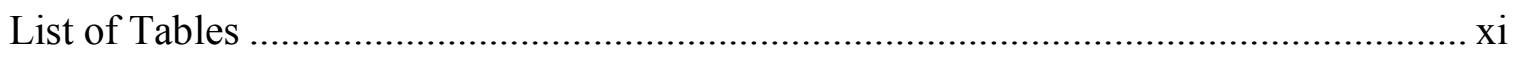

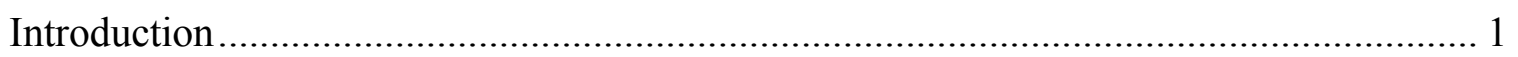

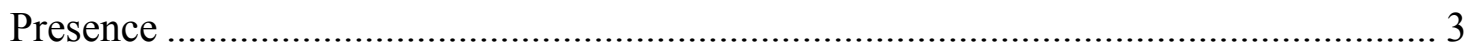

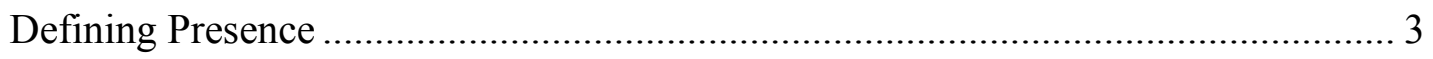

Embodied Cognition and Models of Presence ........................................................ 5

Representation of the Environment and the Body ........................................... 8

Neural Representation of Space and the Body .............................................. 9

Recoding the Representation of Space ..................................................... 10

Recoding the Representation of the Body ................................................... 12

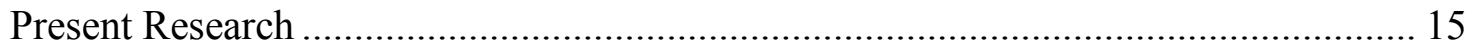

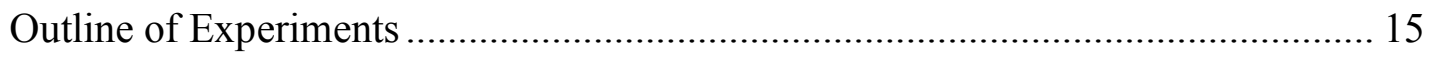

Experiment 1: Asymmetries in left- and right-hand pointing .................................. 17

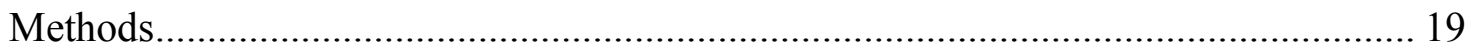

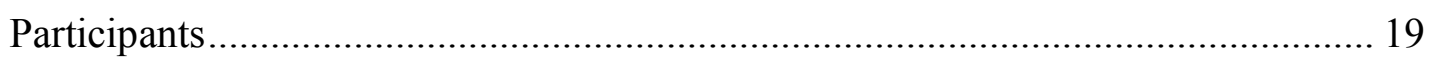

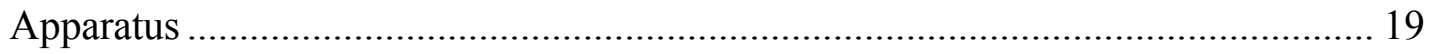

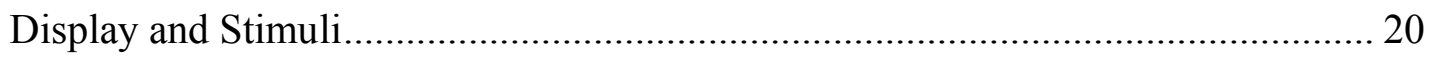




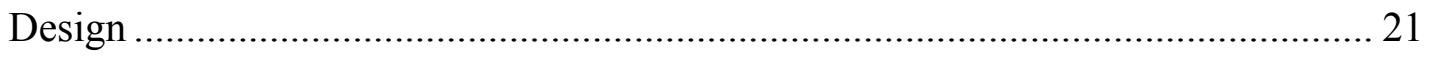

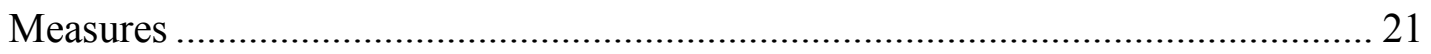

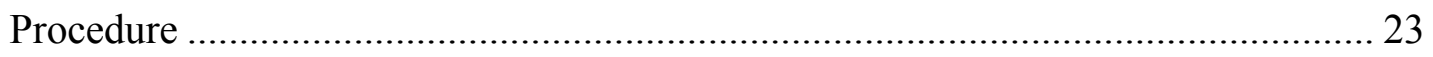

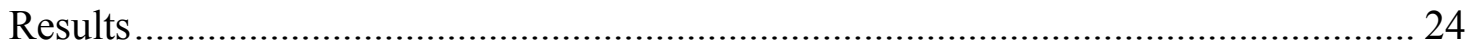

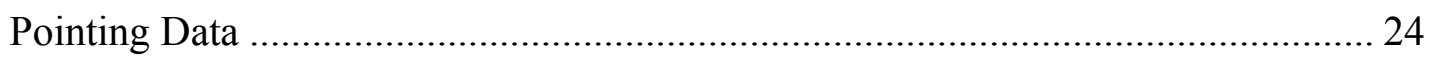

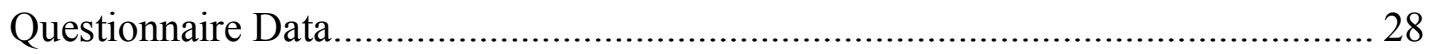

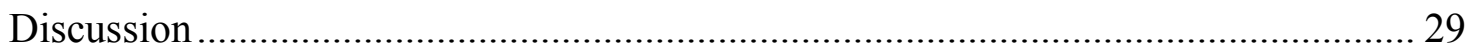

Pointing kinematics in VR reflect natural pointing behaviour .................................. 29

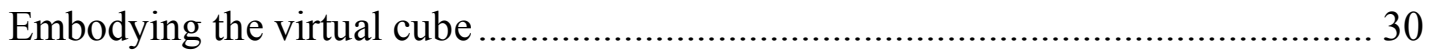

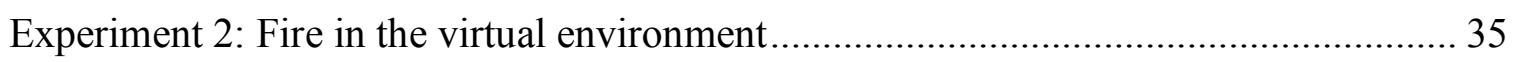

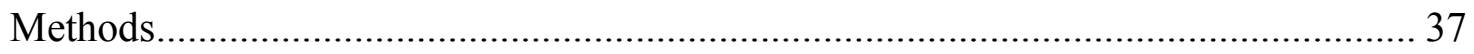

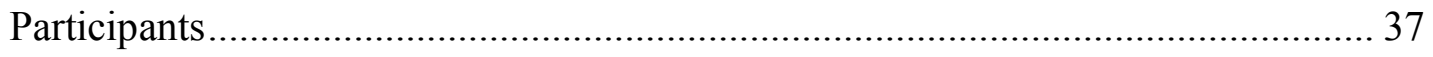

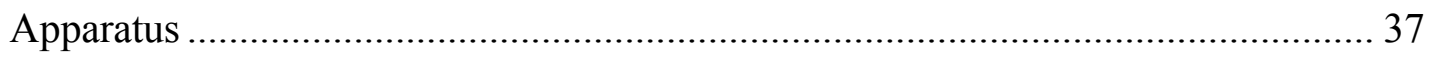

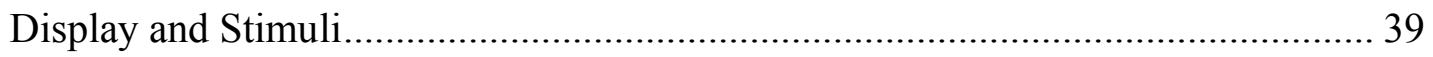

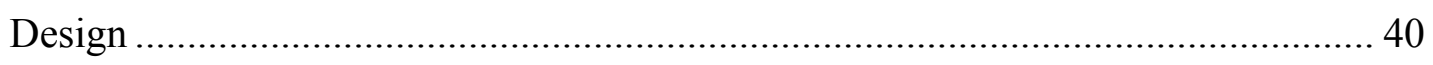

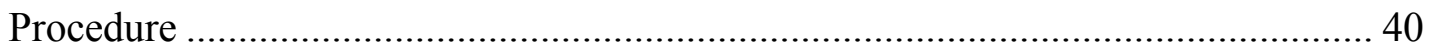

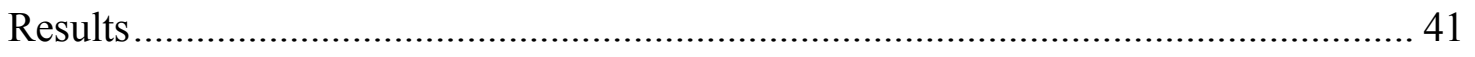

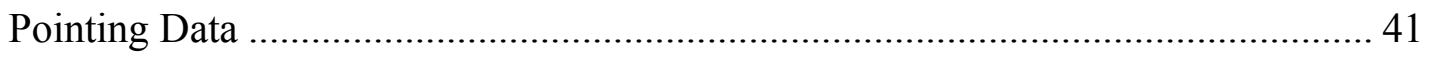

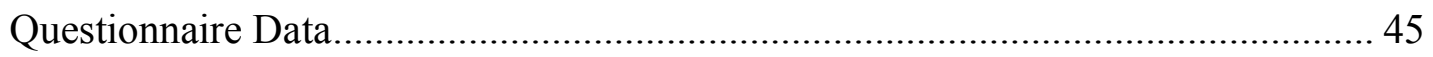

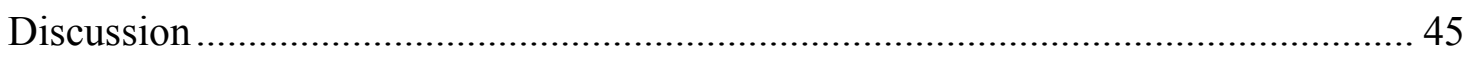

Fire affects movement planning but not execution..................................................... 45

Implications for embodied presence from Experiment 2.......................................... 46 
Summary

Experiment 3: The effects of environment versus effector on embodied presence

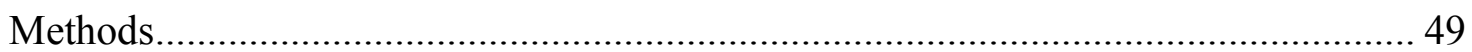

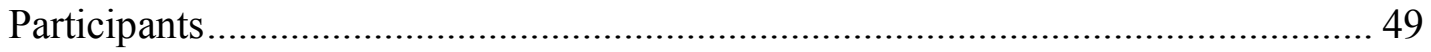

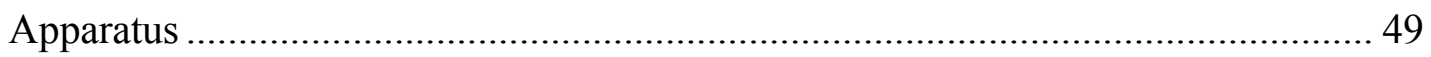

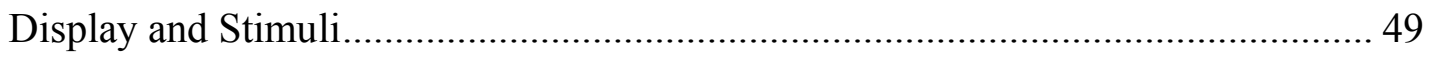

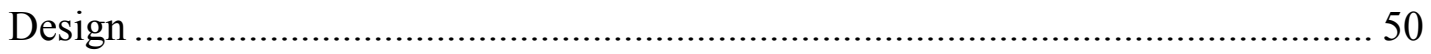

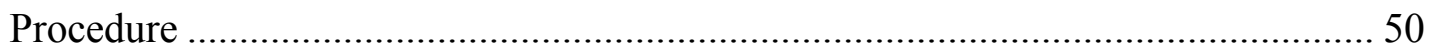

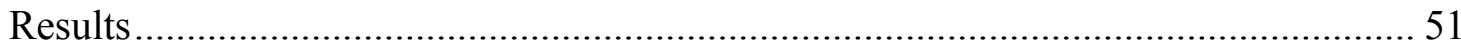

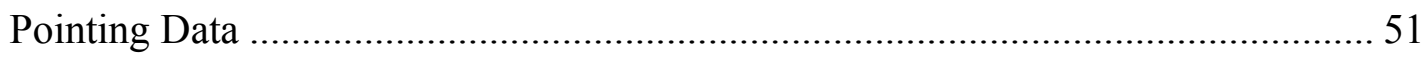

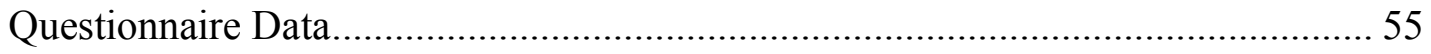

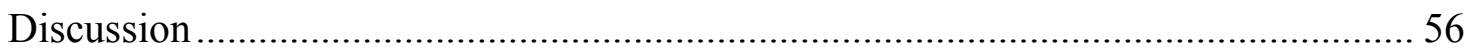

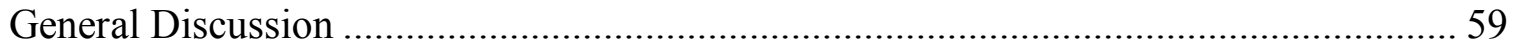

A serial relationship between environment and effector .......................................... 61

Environment and effector in other models of presence …….................................... 63

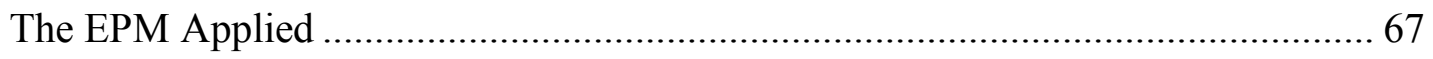

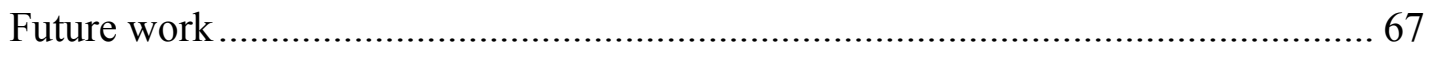

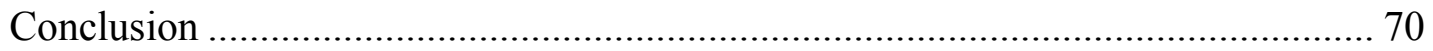

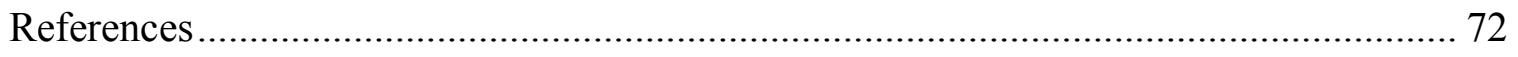

Appendix A: Experiment documents .......................................................................... 77

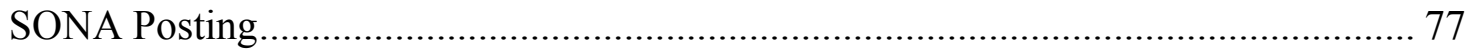


Embodied presence in virtual reality vii

Informed Consent Form ................................................................................. 78

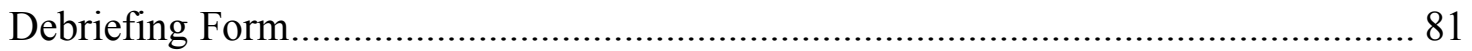

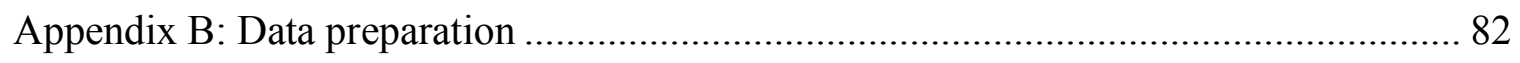

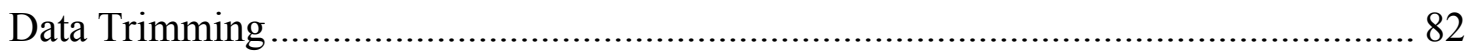

Calculations of Euclidean Distance and Accuracy .............................................. 84

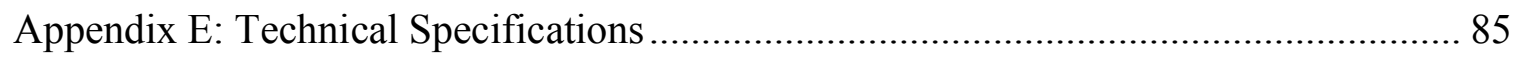

Alterations to Leap Motion tracking protocol ….................................................. 85 


\section{TABLE OF FIGURES}

Figure 1. The Embodied Presence Model, where the experience of embodied presence (mind) is supported by two factors: environmental (world) and effector (body)

Figure 2. Representation of the hypothesized EPM, based on the underlying framework of embodied cognition, which proposes a relationship between mind (presence) body (effector) and environment. 16

Figure 3. Screen capture of the display showing the virtual desk, blue circle for the start position, the cube representing the participant's hand, and one of the small black circle targets. The background shows the gray checkered floor and blue sky at the horizon. 21

Figure 4. Trial sequence diagram, time intervals measured in ms.

Figure 5. Mean travel times (top) and liftoff time (bottom) in ms for the left and right hands at Targets 1-5. Error bars represent 95\% confidence intervals for the comparison of Hand as per Hollands \& Jarmasz, 2010. 26

Figure 6. Mean Percent Hit Rate to all targets by Left-hand (solid) vs. Right-hand (dashed). Error bars represent 95\% CIs for the comparison at Hand as per Hollands and Jarmasz, 2010.

Figure 7. Mean responses to post experiment questionnaire where "- - _" signified strong disagreement and " +++ " signified strong agreement and error bars represent standard deviation of the item mean.

Figure 8. Simplified model of relationship between motor planning and motor control. 36

Figure 9. High fidelity representation of the hand as well as the collision volume (red dot) attached to the index finger (not visible during the experiment). 38

Figure 10. Trial sequence diagram, time intervals measured in ms. 
Figure 11. Mean liftoff times to all targets for the None, Near, and Far conditions of Fire. Error bars represent $95 \%$ CIs for the comparison at Fire as per Hollands \& Jarmasz, 2010 .. 42

Figure 12. Mean travel times to all targets by Fire Location. Error bars represent $95 \%$ Cis for the comparison at Fire as per Hollands and Jamasz, 2010.

Figure 13. Mean Percent Hit Rate to all targets for the None, Near, and Far conditions of the Fire. Error bars represent 95\% CIs for the comparison at Target as per Hollands and Jarmasz, 2010).

Figure 14. Mean responses to post experiment questionnaire where "- - -" signified strong disagreement and " +++ " signified strong agreement and error bars represent standard deviation of the item mean.

Figure 15. Cube representation of fingertip, note that the hand is visible in Figure for demonstration purposes but was not visible to participants during experiment)

Figure 16. Mean liftoff times to all targets by Fire and Hand Representation. Error bars represent 95\% CIs for the comparison at Hand Representation as per Hollands and Jarmasz, 2010.

Figure 17. Mean travel times to all Targets to all targets by Fire and Hand Representation. Error bars represent $95 \%$ CIs for the comparison at Fire as per Hollands \& Jarmasz, 2010 .. 53

Figure 18. Mean Percent Hit Rate by Fire and Hand Representation. Error bars represent 95\% CIs for the comparison at the Hand Representation as per Hollands and Jarmasz, 2010.

Figure 19. Mean responses to post experiment questionnaire where "- - -“ signified strong disagreement and " +++ " signified strong agreement and error bars represent standard deviation of the item mean. 
Figure 20. Update to the EPM with findings from Experiment 3 mapped against the simplified model of motor control presented in Experiment 2. A lack of interaction between environment and effector on pointing movement kinematics suggests environment and effector are independent influences on embodied presence.

Figure 21. A serial version of EPM with a depiction of the motor control model as supporting evidence presented at bottom in square boxes.

Figure 22. Histogram showing distribution of recorded final depth positions in cm (End_X) of the motion controller, negative numbers represent positions behind the start position and positive numbers represent final positions between the start position and targets, where targets are arranged at 27, 29, and $30 \mathrm{~cm}$ away from start. 


\section{LIST OF TABLES}

Table 1. Omnibus effects for 2 (Hand) x 5 (Target) repeated measures ANOVAs for pointing movement liftoff time and travel times measured in ms.

Table 2. Omnibus effects for 2 (Hand) x 5 (Target) repeated measures ANOVA for landing accuracy calculated as percent hit rate per hand at each target.

Table 3. Omnibus effects on liftoff times in the 3 (Fire Location) x 5 (Target) repeated measures ANOVA

Table 4. Omnibus effects on travel times in the 3 (Fire Location) x 5 (Target) repeated measures ANOVA.

Table 5. Omnibus effects on Percent hit rate for the 3 (Fire Location) x 5 (Target) repeated measures ANOVA.

Table 6. Omnibus effects on liftoff times for the 2 (Hand Representation: cube vs. hand) $\mathrm{x} 2$

(Fire: none vs. near) x 5 (Target) repeated measures ANOVA.

Table 7. Omnibus effects on travel times for the 2 (Hand Representation: cube vs. hand) $\mathrm{x} 2$

(Fire: none vs. near) x 5 (Target) repeated measures ANOVA.

Table 8 Omnibus effects on Percent hit rate for the 2 (Hand Representation: hand vs. cube) x 2

(Fire: none vs. near) x 5 (Target) repeated measures ANOVA.

Table 9. Factors of environment, effector, and their interaction in other models of presence in the literature. 


\section{EMBODIED PRESENCE: EVIDENCE FOR EMBODIMENT OF EFFECTORS WHEN POINTING IN VIRTUAL ENVIRONMENTS}

The human brain is hardwired to integrate and represent information from our surroundings and the possible actions we might take. We also construct mental representations of the actions of others, including motor simulations of observed action (Jeannerod, 2001; Rizzolatti, Fadiga, Gallese, \& Fogassi, 1996) to mirrored brain activation for witnessed pain in others (Jackson, Rainville, \& Decety, 2006). Through mental simulation and suspension of disbelief (Coleridge, 1871; as cited in Lombard \& Ditton, 1997; Hofer, Wirth, Kuehne et al., 2012; Wirth, Hartmann, Bocking et al., 2007), we can be transported into fictional worlds of alternate realities often by simply reading a compelling narrative (Green \& Brock, 2000). Today, this transportation into narrative worlds can be enhanced through virtual reality technologies. We have come from fireside storytelling to radio dramas and feature-length films, to fully immersive virtual reality experiences. But what is the consequence of being transported into these increasing believable worlds, and how do we articulate the experience of "being in them"?

During video game play the cortical areas associated with performing the actions of the character on the screen are activated in the player (Klasen et al., 2012). Motor simulations of simulated actions in the game are taken as evidence of being immersed, or transported, into the game world. Given that the brain simulates the experiences represented in two dimensions on a screen, increasing the fidelity of fictional representations should preserve or even enhance mental simulation. For this reason, fully immersive virtual reality (VR) systems have become the focus of much research. Researchers can use VR technology to present participants with impossible worlds or situations that would be costly or dangerous to test within. For example, 
VR has been used in therapeutic settings such as phobia intervention (e.g., Carlin, Hoffman, \& Weghorst, 1997). VR also provides a safe and cost-effective alternative to large-scale simulators for training in domains such as flying aircraft or operating remote vehicles. With the potential for real-world applications to come out of VR research, it is imperative to understand the nature and extent to which virtual worlds are experienced as real. The exploration of this question has been taken up by a field of research on the concept of presence.

Many definitions of presence have been offered (see Skarbez, Brooks, \& Whitton, 2017 for a review) and have focused on describing the subjective feeling of being present in a virtual environment (i.e., "being there”, Heeter, 1992; Slater, Usoh, \& Steed, 1994; Witmer \& Singer, 1998). With a few exceptions, presence in virtual environments has been measured using selfreport questionnaires that are administered after participants have been exposed to a virtual environment. A few standard questionnaires have emerged (e.g., Slater Usoh Steed, 1994; Witmer \& Singer, 1998), but researchers tend to favour their own questionnaires to index participants' subjective sense of presence and the field is still lacking a definitive measure of presence. Moreover, although some models of presence have been offered, these models differ depending on the discipline from which they originate. Early models focused on the technological capabilities and the subsequent fidelity of stimuli that the VR system could provide to the observers (e.g., Bystrom et al., 1991; Schubert, Friedmann, \& Regenbrecht; 1999, 2001; Sheridan, 1992). More recent models have taken the perspective that presence should be considered as a consequence of observer-level factors such as one's predisposition to suspension of disbelief or mental imagery (e.g., Hofer et al., 2012; Weibel \& Wissmath, 2011) and some have begun including new constructs such as social interaction as technology advanced have made this possible (e.g. Sivunen \& Nordback, 2015; Skarbez et al. 2017). To date, however, 
none has incorporated the current neuropsychological understanding of how the human brain represents and interacts with the environment around the individual.

In the present work, a model of presence called the Embodied Presence Model (EPM) is proposed. The EPM is informed by the neuropsychological perspective and an embodied cognition perspective. The EPM is examined with behavioral data from three experiments. The case is made that embodied presence in VR is supported by the same mechanism supporting our interaction with the natural world: a representation of the environment and a representation of the effectors with which we interact with the world. Although there is an abundance of self-report evidence for many theorized distinctions in the concept of presence, very few studies report behavioural measures of presence. Further, there is little consistency in the breakdown of presence into its constituent parts. Most researchers agree that presence is a multi-component construct, and across different models of presence two themes emerge: Presence involves both a sense of being in a believable place and being able to interact with that place. The EPM collects these themes into the aforementioned supporting components of environment and effector. Evidence for the EPM is presented by examining the experiment data as it relates to characteristics of natural interaction behaviours (Experiment 1), models of motor control (Experiment 2) and additive factors logic (Experiment 3).

\section{PRESENCE}

\section{Defining Presence}

The term presence comes from telepresence which was first used by Minsky (1980) to describe a teleoperator's sense of being in a remote location when using tools to interact with said remote location. Sheridan (1992) coined the term "virtual presence" to articulate the sense of being in an environment that was entirely created by a computer, thus beginning the line of 
inquiry into how presence is supported by the interaction between virtual environments and the human sensory system. Presence is commonly described as a sense of "being there" (Slater, Usoh \& Steed, 1994), but formal definitions can vary in nuance. For example, Biocca (1997) in his seminal paper, "The Cyborg's Dilemma", argued that a person can only experience being in one of the physical environment, the virtual environment, or the imaginal environment. For Biocca, presence is a moving target defined by whichever environment in which the observer's experience of being physically located is most compelling, where shifts in presence between environments are described as arrival and departure (Kim \& Biocca, 1997). Lombard and Ditton (1997) define presence as “an illusion of non-mediation", where emphasis is placed on a whether a virtual environment provides compelling enough cues such that the illusion of being located in the virtual environment goes undetected. Slater (2018) notes that presence is indeed an illusion of perception rather than cognition, while others liken presence to selective attention where one's experience is selectively focused on virtual stimuli over and above stimuli from the natural environment (Witmer \& Singer, 1998).

Presence has been linked to the concept of immersion. Lombard and Ditton (1997) identified immersion as one of the elements involved in presence, where immersion is used to describe one's sense of being engrossed in a narrative, similar to the idea of suspending belief (coined by Coleridge, 1817; as cited in Hofer et al., 2012; Lombard \& Ditton, 1997; Wirth et al.; 2007). In a similar vein, Witmer and Singer's (1998) selective attention account of presence evoked the concept of immersion in the way it described the observer's focus being consumed by technologically presented information. A more formal definition of immersion comes from Slater and Wilbur (1997) who state that immersion reflects the extent to which an observer's sensory system receives inputs that are mediated through technology rather than originating in the natural 
world (see also Bowman \& McMahan, 2017). Through this operationalization, immersion became a quantifiable construct, defined in terms of factors such as visual fidelity, number of audio channels, and degrees of freedom for movement sensors.

Although presence and immersion are linked, these two constructs can be differentiated. As described by Slater and Wilbur (1997), presence occurs when the cues from the virtual environment are highly immersive and more engaging than those from the natural environment. Immersion is a quantifiable measure of sensory inputs delivered by technology. Presence is the subjective experience of "being there". Slater (2018) has noted, however, that there should be a predictable increase in the experience of presence with an increase in the immersive capabilities of a VR system. A similar relationship between immersion and presence has been suggested by researchers examining presence from an embodied cognition perspective (e.g., Schubert et al., 1999, 2001).

\section{Embodied Cognition and Models of Presence}

Embodied cognition is a perspective in psychology in which thought is situated in realworld circumstances and involves our current and remembered experience of action and consequence in the world around us. According to Gibbs (2006, p. 12) "Experiences of the body are represented as ideas in the mind, and the body provides valuable resources for off-loading cognition such that mind is distributed across brain, body, and world interactions." This perspective is counter to the classic information processing model of cognition where the mind is viewed as a computer processor and the body is viewed as computer peripherals. Rather, embodied cognition provides a theoretical basis for observed interactions between mind and body such as the influences that motor activity can have on perception, or that perception of the environment can have on motor planning. In contrast, Skarbez et al. (2018) points out that the 
term embodiment in the computing literature refers to the experience of owning a body, or avatar, in a virtual environment. In the present work, I propose the Embodied Presence model (EPM) in which it is assumed that presence is an embodied cognitive process in the classic psychology sense, wherein this embodied cognitive process supports the computing view of embodiment such that observers feel ownership of their virtual body. On this view, the relationship between mind, body, and environment is fundamental to the experience of an embodied presence in virtual reality (see Figure 1). A central premise in the EPM is that this embodied presence is a direct result of interactions between one's surroundings and one's self. The EPM provides a framework to define and test the relationship between two supporting factors asserted above: a robust representation of the environmental and of the effectors.

In a recent article to introduce the experimental potential of VR technology to psychologists Pam and Hamilton (2019) outline the importance of providing participants with

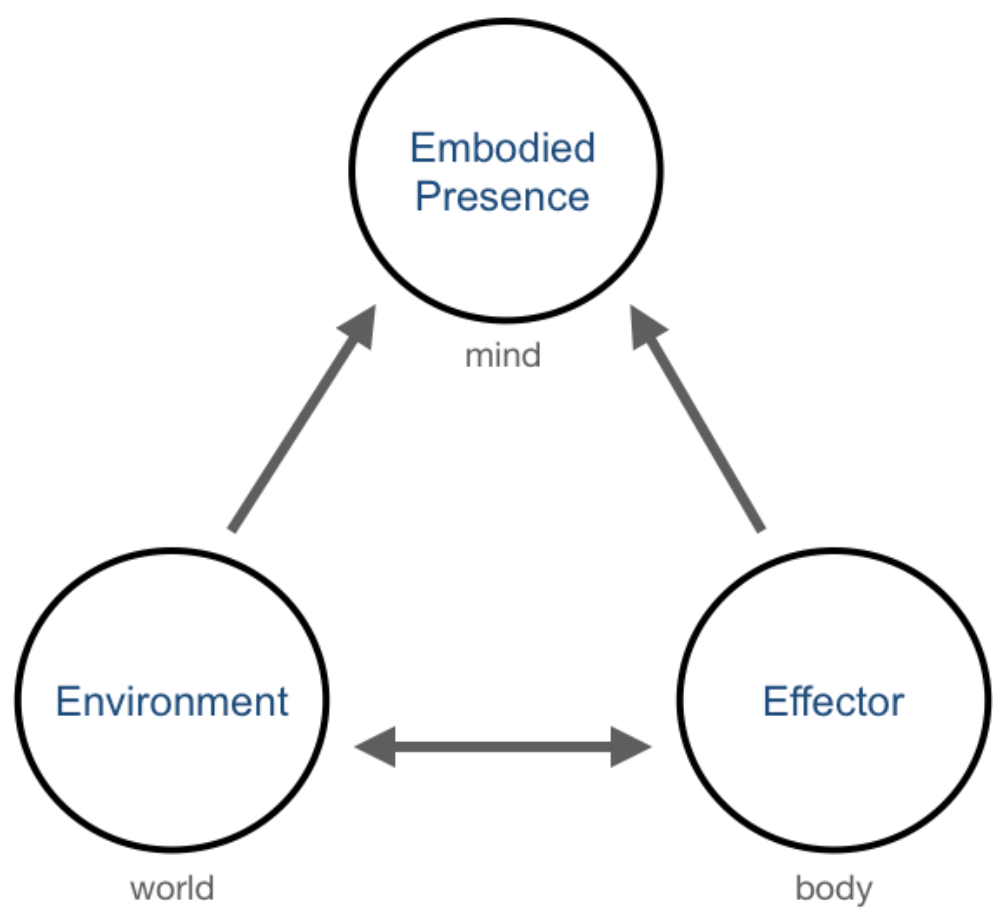

Figure 1. The Embodied Presence Model, where the experience of embodied presence (mind) is supported by two factors: environmental (world) and effector (body). 
compatible visual and motor stimuli in order to create a realistic testing environment. Indeed, the idea that the ability to interact in VR is fundamental to establishing a sense of presence has long been made by researchers (e.g., Sheridan, 1992; Wirth et al., 2007; Witmer \& Singer, 1998; Zahorik \& Jenison, 1998; see also Shuemie, Van Der Straaten, \& Krijn et al., 1999 for a review). Schubert et al. (1999, 2001) conducted a multi-study factor analysis on user experiences obtained from across a variety of virtual environments. The factor analyses revealed two presence factors: involvement and spatial presence. Involvement describes the degree to which, through suppression of the natural environment, one's attention is directed to information within the virtual environment for subsequent interaction. Spatial presence describes the degree to which a person mentally constructs and experiences themselves as being located within an environment. Judgements about the realness of the virtual environment emerged in the second study as a third factor predicting presence. Other factors of presence were revealed in Shubert et al.'s work, most of which loaded separately onto an immersion construct. Using a path analysis these researchers provided some of the first reliable evidence that these immersion factors predict spatial presence. The best predictors of spatial presence were factors surrounding whether the virtual environment provided dramatic or emotional events for the observer and whether users could interact with the virtual environment. Two of the three factors that predict presence in the Schubert et al. model align with Witmer and Singer's (1998) suggestion that presence requires both involvement and immersion (spatial presence, for Schubert et al.).

Wirth et al. (2007) proposed a model of spatial presence to account for the experience of "being in another location" through media other than VR (e.g., books, film). Wirth et al. define spatial presence as a "conviction of being located in a mediated environment" (p. 495). On this view, spatial presence is supported by any media that provide a way to experience a sense of 
being physically located in the environment portrayed (i.e., self-location) and the perception of possible actions in the environment. Although not an entirely new take on presence, Wirth et al. explicitly include suspension of disbelief (Coleridge, 1817; cited in Hofer et al., 2012; Lombard \& Ditton, 1997; Wirth et al. 2007) as a requirement for the emergence of presence.

The Wirth et al. (2007) model highlights how important it is for a virtual environment to be mentally constructed in a fully rendered, interactable manner in order for people to experience a sense of presence. Wirth et al. describe this manner of environmental representation as providing a possibility for exploration and elaboration of the constructed environment. As discussed in the following section, the experience of presence in VR also requires a robust representation of the self which includes some way to interact with the world. The following section unpacks the neurophysiological mechanisms that support both of these representations in the natural world and how these representations have been examined in VR.

\section{REPRESENTATION OF THE ENVIRONMENT AND THE BODY}

In the EPM, presence is supported by environmental and effector representations. The environmental factor is the extent to which a believable virtual space is mentally constructed via the same perceptual cues experienced in the natural world. The effector factor describes the extent to which the virtual manipulators presented in the VR become represented, or adopted, as part of one's own body. This distinction between environmental and effector factors in the EPM is support by research showing that physical space and a person's body are represented differently in the brain. Further, the representations of physical space and the body are both subject to recalibration depending on perceptual cues. The re-codable nature of our representation of both the environment and the body is what supports the experience of embodied presence in VR. 


\section{Neural Representation of Space and the Body}

Environmental presence is supported by the same mechanisms that lead to a sense of being physically present in natural environments: the representation and dynamic recoding of space by the brain. Physical space is neurologically represented by its relationship to the body in three distinct categories: pericutaneous space, peripersonal space, and extrapersonal space. Pericutaneous space (Rizzolatti, Scnadolara, Matelli, \& Gentilucci, 1981b) begins as the skin and extends to just the very near peripersonal space, almost as though it were a "gelatinous mold surrounding the body" (Graziano, Yap, \& Gross, 1994). Slightly farther from the body, peripersonal space is that which can be reached, it extends out from the body to an approximate arm's length radius (Berti \& Frassinetti, 2000). Peripersonal space is coded dynamically as a function of what can be reached, and therefore expands with tool use (e.g., Iriki, Tanaka, Iwamura; 1996). Extrapersonal space is all space that is left beyond one's reach.

Early neural recording studies from Rizzolatti et al. (1981a; 1981b) demonstrated evidence of the neural distinction between extrapersonal and peripersonal space in the macaque monkey brain. These researchers provided some of the first evidence that the brain preserves a codified distinction between types of space near the hand that the way space is represented in the brain is a multisensory phenomenon. Neurons in the macaque brain were located by presenting a variety of stimuli along different trajectories or in static positions both near and far from the monkey's body and watching for neuronal response. A clear distinction was found between neurons specialized to process visual stimuli far from versus near the monkey's body. These neurons fell into one of two classes: Pericutaneous neurons responded to stimuli a few centimeters from the body, and peripersonal neurons responded to stimuli within space which the monkey could reach. Most critically, pericutaneous neurons that code for the representation of 
the body responded to both visual and tactile stimulation. This discovery of a bimodal (visuotactile) representation of the body has spurred countless examinations of body representation as a multimodal, bottom-up phenomenon.

It is now known that multisensory coding of the body in space is informed by visual, tactile, and proprioceptive inputs and is often referred to as the body schema (Gallagher, 2006). The body schema serves as a source of information about where the body is in space and how it is moving by constructing and updating an online representation based on multisensory inputs. It is this dynamic shifting of body schema that allows us to operate in differently sized spaces and to support the use of tools by shifting the distinction between reachable and non-reachable space. A large body of literature describes this dynamic recoding of the representation of space that arises with tool use and holds implications for the types of recoding that may happen when we receive perceptual inputs not only from physical tools, but from virtual tools or environments.

\section{Recoding the Representation of Space}

In order to interact with an environment, virtual or otherwise, it is necessary that not only the physical space and objects in one's surrounding be represented by the brain, but that these representations be malleable enough to support changing contexts. Tool use is a well-studied example of a situation when the brain needs to recode the representation of surrounding space in order to interact with the environment. This was demonstrated in the Macaque monkey by Iriki et al. (1996) who localized populations of neurons with receptive fields that are located on the hands. The monkeys were trained to retrieve small objects on a table using a rake tool, and after training the experience of using the rake resulted in the visual receptive fields for those neurons extending from the hand to the space along the length of the rake. These neurons responded to visual stimuli appearing near the rake as though it were the hand. These neurons quickly 
'shrank' back to the monkey's hand when the rake was no longer in grip. That is, the brain recoded the visual representation of pericutaneous space to encompass the rake until it was no longer needed, at which point the standard representation of peripersonal space was reinstantiated to that space directly surrounding the hand.

Evidence from neurological patients has also demonstrated recoding of peripersonal and extrapersonal space, most notably in a study of patient PP's case of visual neglect (Berti \& Frassinetti, 2000). Patient PP suffered damage to the right hemisphere and was unable to perceive information about the space in the opposite (left) visual field, despite having a fully intact peripheral system able to detect and transmit visual stimuli to the right hemisphere. In short, PP showed a diminished ability to attend and process visual information presented in the left hemi-field of peripersonal space. When a line bisection task was moved out to extrapersonal space with the use of a laser pointer, PP's performance improved relative to a standard paper and pencil task. When PP performed the same line bisection task in extrapersonal space with a pointing stick instead of a laser pointer his performance declined back down to the levels seen on a standard paper and pencil task. Holding and using a pointing stick provided enough tactile and proprioceptive cues about the length of the stick to prompt PP's brain to recode the extrapersonal space as peripersonal space.

As was demonstrated in monkey's brains by Iriki et al. (1996), the neurological representation of peripersonal space in PP's brain was recoded to extend outward when he picked up the pointing stick. Studies of other neurological patients have also demonstrated the brain's ability to recode peripersonal space as pericutaneous space (e.g., Brown, Kroliczak, Demonet, \& Goodale, 2008). When patients were asked to estimate the size of and grasp a variety of small plastic blocks presented at different locations on a vertical board their 
performance was dramatically worse than that of healthy controls. However, if the patients were asked to place one hand next to the blocks and then use their other hand to estimate size by indicating with the thumb and forefinger then their performance improved. Placing blocks within pericutaneous space likely allowed the size-processing tasks to benefit from the recruitment of the additional processing resources in areas that represent the space near the hands rather than relying on the damaged processing resources responsible for representing peripersonal space. This dynamic recoding of space and the illusion described in the next section both suggest that the brain is ready and able to recode what is considered to be near or even part of the body in space.

\section{Recoding the Representation of the Body}

Just as it is useful to understand the manner in which the brain represents physical space for environmental presence, it is also important to understand how the brain represents the body in a manner that can support effector presence. In VR, effector presence requires that virtual manipulators (e.g., avatar hands or tools) be adopted as part of the body. The neurophysiological mechanism that supports the brain's representation and ownership of body parts is well described by demonstrations of the rubber hand illusion. The rubber hand illusion demonstrates that visual and tactile stimulation are combined to create a sense of ownership over one's hand, or in the case of the illusion, a rubber hand. This illusion shows that the brain can adopt foreign objects as body parts. Arguably, adopting virtual representations of the body should be no different.

Botvinick and Cohen (1998) demonstrated the rubber hand illusion using a paintbrush and a realistic looking dummy left hand and arm. Each participant's left hand was placed out of sight behind a vertical board while the rubber hand was placed in a location plausible to be the participant's own left hand. A white sheet was draped over the participant's shoulder and the 
elbow end of the dummy arm to mask any visual cues that the dummy hand was not attached to the body. The experimenter then simultaneously stroked the fingers of the rubber hand and the participant's hidden hand in the same direction with paintbrushes. After ten minutes of paired visual and tactile stimulation participants completed a questionnaire and gave an open-ended description of their experience during the experiment. All participants agreed strongly with the questionnaire item "It seemed as if I was feeling the touch of the paintbrush in the location where I saw the rubber hand touched".

Botvinick and Cohen's (1998) first experiment highlighted the malleability of participants' ownership of their left hand such that they adopted a foreign hand in place of their own. A second experiment quantified this malleability with measurements of proprioceptive drift. Proprioceptive drift is obtained by asking participants to close their eyes and use their right hand under the table to point to where they thought their left hand was. Participant's estimates of left-hand location were displaced rightward to a location much closer to the rubber hand than their own left hand. Furthermore, proprioceptive drift varied as a function of duration of illusion experience. Those participants who experienced the illusion for longer showed more displaced reaches than those who experienced the illusion for a shorter portion of the experiment. The Rubber Hand Illusion represents some of the strongest evidence of the multisensory perceptual process that leads to a sense of ownership over one's body and has been replicated and extended in a variety of settings, including virtual environments.

In a replication of the rubber hand illusion, Ijsselsteihn, de Kort, and Haans (2006) used a projector setup to replace the rubber hand with a virtually presented rubber hand on the table in front of participants. The researchers also included an unmediated (technology-free) control condition that replicated the original experiment and findings from Botvinick and Cohen (1998). 
Tactile stimulation to the participants' own hands followed the standard procedure in all conditions: participants' left hands was placed away from view and stroked with a paintbrush in synchrony with the visual paintbrush stimulating a rubber hand of different reality conditions. Every participant experienced the rubber hand illusion more strongly in a purely virtual reality setting, where both the rubber hand and paintbrush appeared virtually, as compared to a mixed reality setting where only the hand is presented virtually while the paintbrush was applied to the actual desk in a position aligned with the virtual rubber hand. Ijsselsteihn et al. suggested that this was an effect of both the visible texture difference between the appearance of the real paintbrush and virtual rubber hand in the mixed-reality condition and the lack of 3dimentionality of both of the virtual conditions.

Ijsselsteihn et al. (2006) found that many participants noted that during the virtual reality condition they began to feel as though the virtual rubber hand they were seeing was merely a projection of their real hand, which was out of sight. In the mixed-reality condition, some participants reported that the virtually displayed rubber hand became more convincing over time and even appeared to "obtain volume" as the stimulation carried on. The researchers concluded that although the illusion is not as convincing in virtual reality settings as the natural setting of the original setup, the fact that it can be induced at all in a virtual setting provides promising evidence that the perceptual system will adopt non-biological artifacts and "effectively integrate the technology as a phenomenal extension of the self' (Ijsselsteihn et al., 2006, p. 463).

Other illusions have been demonstrated with virtual bodies. For example, a "body swap" illusion was performed by Petkova and Ehrsson (2008) using a mannequin instead of a rubber hand. Later, Guterstam, Abdulkarim, and Ehrsson (2015) demonstrated a whole-body invisibility illusion in VR by removing any rubber hand or mannequin from the equation and applying visual 
stimulation to empty space. To induce the whole-body invisibility illusion, participants were shown a virtual display of the empty space which was described to them as a first-person view of an invisible body oriented in the same position as their own body through a head-mounted VR display. The view of the virtual world was such that when participants were instructed to look down toward the floor it appeared as though their body were invisible. In sum, the rubber hand illusion and the variety of other body ownership illusions demonstrate the malleability of a sense of ownership of one's body, which explains why people are able to adopt and use virtual manipulators so easily in VR and other mediated environments such as video games or remote operating environments. With the right cues, the brain can adopt a virtual manipulator as an owned body part. The dynamic recoding of space reviewed in the previous section and body ownership illusions described here provide the theoretical basis for the EPM, and in particular, the notion that presence emerges as the result of interactions between the brain's representation of environment and body.

\section{PRESENT RESEARCH}

In the EPM, presence is considered to be composed of two contributing factors: an environmental factor and an effector factor. The purpose of the present research was to examine the role of these two factors on supporting a sense of presence. For the present work, embodied presence is assumed to be affected by one's ability to interact within a virtual environment. In order to interact with an environment, a robust representation of both the environment and the self must be intact. Both of these tenets are represented in the EPM (see Figure 2).

\section{Outline of Experiments}

Three experiments were conducted to examine the relationship between environment and effector components of presence and their influence on the subjective experience of embodied 


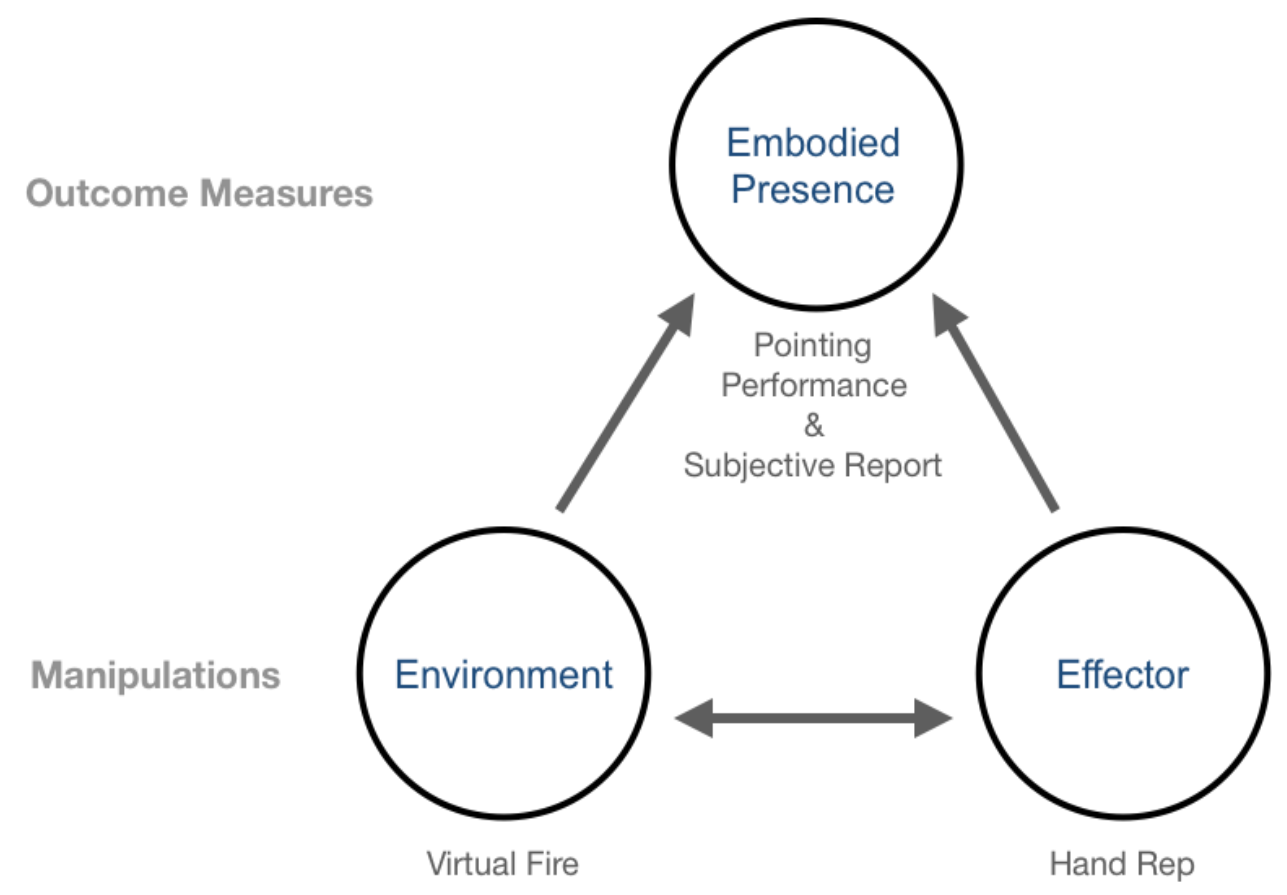

Figure 2. Representation of the hypothesized EPM, based on the underlying framework of embodied cognition, which proposes a relationship between mind (presence) body (effector) and environment.

presence. Experiment 1 established a paradigm in which participants were asked to make pointing movements toward small targets presented on a virtual table. The results of Experiment 1 provide evidence that participants' pointing behaviours in a virtual environment are consistent with those observed in natural settings, suggesting that participants' representations of their body and its effectors were intact. Experiment 2 demonstrated that alterations to the virtual environment caused distinct effects on participants' pointing behaviours, highlighting the importance of participants' representation of the environment. Experiment 3 provides evidence that alterations to the environment versus the effector produced separate effects on participants' pointing behaviours, supporting a distinction between the role of environmental and effector factors in supporting embodied presence. 


\section{EXPERIMENT 1: ASYMMETRIES IN LEFT- AND RIGHT-HAND POINTING}

Experiment 1 established a VR pointing task that was used throughout this thesis. The pointing task was modelled after tasks used in the motor learning literature in order to compare virtual pointing effects to those found in studies of natural pointing behaviours. Specifically, Experiment 1 leveraged well-documented asymmetries in movement characteristics between the left and right hands. Studies conducted in natural environments have demonstrated differences in speed to initiate versus complete pointing movements between the hands, as well as differences in pointing accuracy. Experiment 1 examined whether these same patterns of left- versus righthand pointing persist in VR when the hands are represented by virtual effectors.

In a classic pointing study, Fisk and Goodale (1985) asked participants to point at targets on a vertical board in front of them with the index finger of both their left and right hands in blocked conditions. The display board contained a central fixation cross flanked horizontally by two targets to the left and two to the right at 10- and 20-degrees visual angel (DVA) from the central fixation marker. Thus, for both hands there were two ipsilateral (same-side) and two contralateral (opposite-side) targets to point to. Targets were small (1 DVA) fluorescent light bulbs presented randomly for either $100 \mathrm{~ms}$ duration or until the reach was completed. The researchers found a substantial difference in the way participants reached for ipsilateral versus contralateral targets. Regardless of hand, on trials when ipsilateral targets were presented participants demonstrated faster liftoff time and a faster overall travel time than on trials with contralateral targets. Further, contralateral targets furthest from each hand showed longer liftoff times than closer contralateral targets. The furthest contralateral targets also took the longest to travel to and had the poorest pointing accuracy of all targets. 
Another characteristic finding of bi-manual reaching was demonstrated by Elliott, Roy, Goodman, et al. (1993), who asked participants to point to small targets using a stylus while manipulating target size, pointing hand, and fast versus slow pointing movement. In trials blocked by hand and target, participants pointed to different circular targets. There was more pointing error for left- than right-hand trials and more error for fast than slow movement trials. A second experiment presented a spatial uncertainty element where participants were asked to point to a circular target presented at 12 degrees visual angle to the left or right of center. This experiment revealed faster liftoff times for left hand than the right hand, but shorter travel times and better pointing accuracy for the right hand. Elliot et al. concluded that the left hand may have demonstrated faster liftoff time because of its connection to the spatially dominant right hemisphere.

The idea that connections to the left- versus right-hemisphere should cause differential effects on reaching has been adopted by many researchers. Boulinguez, Nougier and Velay (2001a, 2001b) demonstrated the same pattern of movement asymmetries between the left and right hands as Elliott et al. (1993). In an examination of perturbations to reach trajectories these researchers also found faster liftoff times for the left- than the right-hand and faster travel times for the right hand as well as a speed advantage for ipsilateral compared to contralateral targets, replicating the findings of both Elliott et al. (1993) and Fisk and Goodale (1985).

In the present research, Experiment 1 was aimed at examining participants' pointing movements to test whether the representation of the body and its effectors remains intact in virtual settings. An intact representation of the body was defined as the degree to which virtual effectors are embodied by participants, where greater embodiment would allow participants to use the virtual effectors to interact with the virtual environment in a manner that is 
indistinguishable from natural environments. It was therefore hypothesized that if participants embodied a virtual cube effector in the virtual environment then their pointing behaviours should express the same asymmetries as those observed in natural conditions. In particular, it was predicted that liftoff times would be faster for left- than right-hands. Conversely, travel times should be faster and more accurate for right- than left-hands (Boulinguez et al., 2001; Elliott et al., 1993). It was also predicted that participants would take longer to reach targets located on the opposite side of the body midline (Fisk \& Goodale, 1985).

\section{METHODS}

\section{Participants}

Participants were 52 undergraduate students recruited from the Carleton University research pool in exchange for $1.5 \%$ bonus toward an eligible psychology course. Four participants had some previous experience using a modern virtual reality headset and one participant was not strongly right-handed. Preliminary inspection revealed that the data for these participants did not differ from the main data set. Participants who wore glasses were given the option of wearing or not wearing their glasses based on the most comfortable and clear viewing experience. Participants were also asked to remove bulky headwear or hairstyles that interfered with a good fit of the VR headset. Participants were required to have a neurological history free from any motor control difficulties or conditions predisposing them to nausea (see Appendix A: Experiment documents). None of the participants became dizzy, disoriented, or uncomfortable during the experiment. One participants' reaching data were discarded due to hardware failure. Fifty-one participants' reaching data remained for analysis.

\section{Apparatus}


The experiment consisted of a virtual environment containing a virtual desk upon which participants reached for targets. The virtual environment was displayed to participants via an Oculus Rift Development Kit 2 VR headset. The headset's proximity to the eyes allowed for total visual immersion with 960x1080 pixel resolution and a 100-degree horizontal viewing angle. The Oculus Rift has tracking technology that follows head movements such that the wearer can move their eyes and head naturally to observe the virtual world without any inconsistencies between perceived head motion and the viewed scene1.

Participants' hands were tracked by having them hold a Razer Hydra game controller. The Hydra uses an accelerometer and magnetic positional tracking to detect the location of the controller with excellent spatial and temporal accuracy. As participants held and moved the controller in the experiment workspace, its position was virtually represented by a small cube that participants were encouraged to adopt as a visual marker of their hand. The experiment program was run by a computer that also was used to collect and store the raw data. The experiment took place in a small room where participants sat in a chair at an ordinary desk. The experimenter was in the room to monitor the experiment while keeping participants' hand movements in sight until practice was complete.

\section{Display and Stimuli}

The virtual environment was a large open space with a blue sky and a gray desk that was positioned to match the height and size of the physical desk in the experiment room. The start position used to end initiate trials appeared on the virtual table as a small blue circle. The virtual hand marker (cube) followed the movements of the hand-held controller and appeared as a floating cube (Figure 3). The virtual desk appeared empty until the experiment began. Each trial 


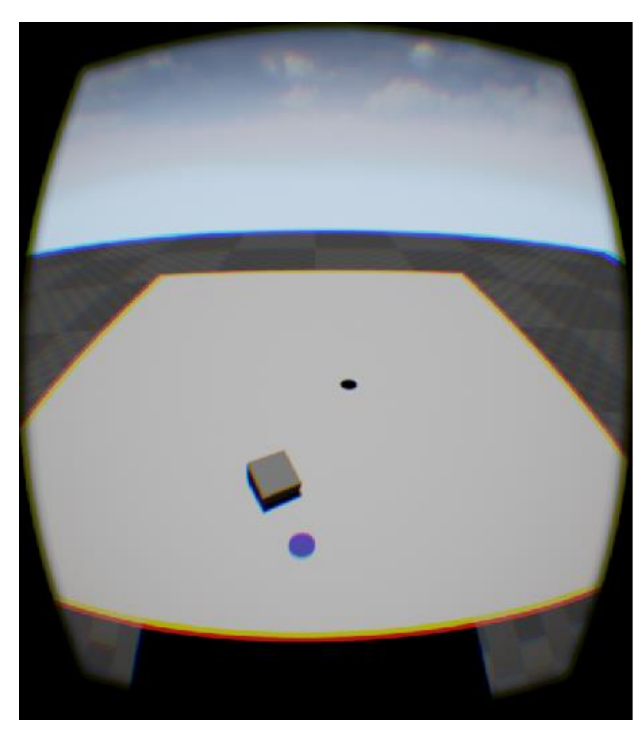

Figure 3. Screen capture of the display showing the virtual desk, blue circle for the start position, the cube representing the participant's hand, and one of the small black circle targets. The background shows the gray checkered floor and blue sky at the horizon.

began with a fixation cross presented centrally on the virtual desk $20 \mathrm{~cm}$ from the participant.

The fixation cross was presented for a random duration between 500 and $1500 \mathrm{~ms}$, after which a target appeared at one of the five potential target locations. Targets remained visible until the either participants completed a full pointing movement toward a target or a 5000ms trial timer expired (see Figure 4). Target locations were configured along a small semi-circle with a central target $30 \mathrm{~cm}$ from the virtual body flanked by two targets to the left and right at 7 and 14 degrees from center on each side. One of these target positions was illuminated per trial.

\section{Design}

Experiment 1 utilized a 2 (Hand: left vs right) x 5 (Target: extreme left, left of center, center, right of centre, extreme right) repeated-measures design.

\section{Measures}

The aim of the experiment was to compare pointing performance between participants' left and right hands in terms of kinematic characteristics. Dependent measures for the pointing task were liftoff time, travel time, and landing accuracy. Liftoff times were measured as the time 


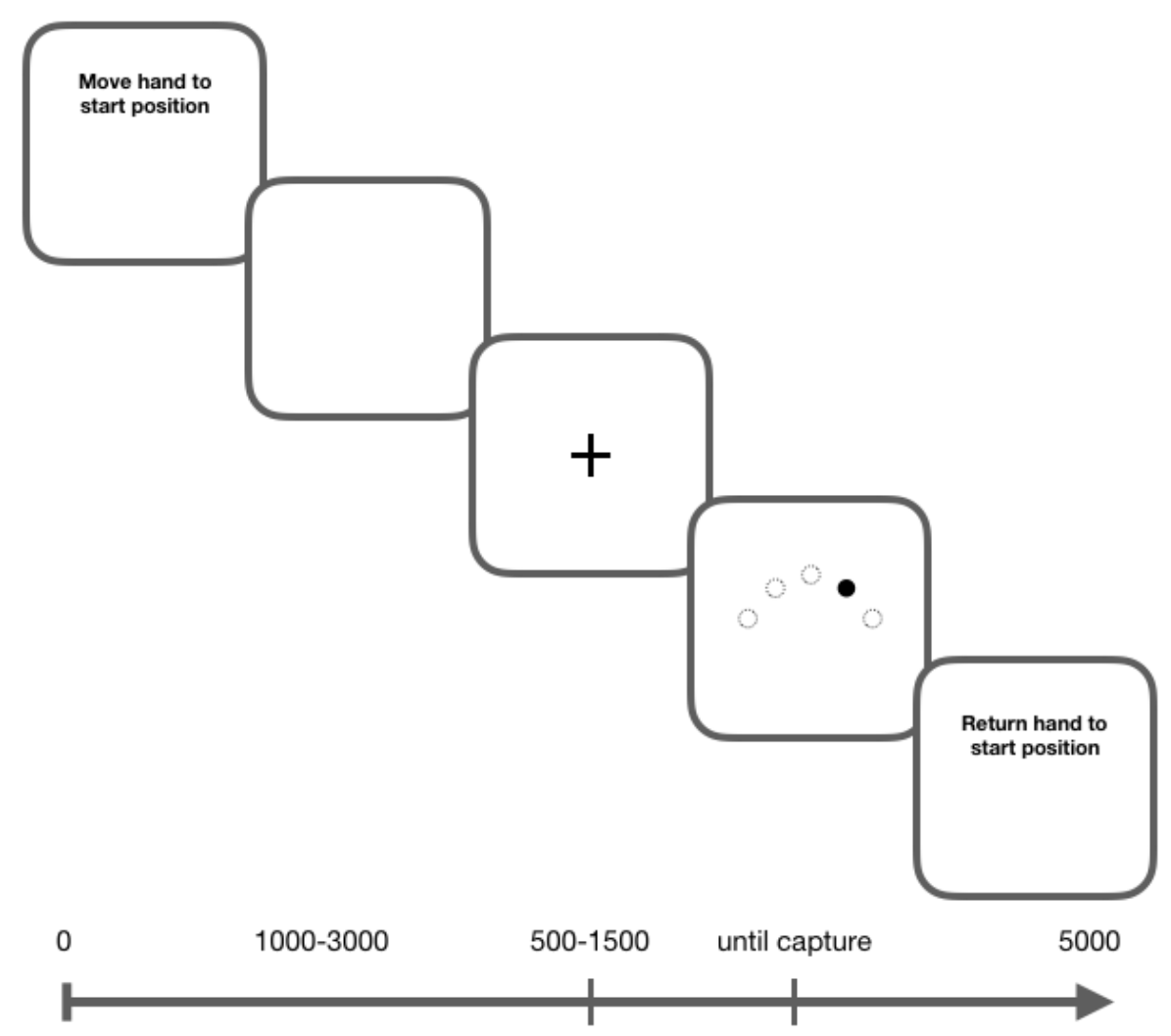

Figure 4. Trial sequence diagram, time intervals measured in ms.

it took participants to initiate a pointing movement after a target had become illuminated. The initiation of liftoff was defined as the moment that the motion controller surpassed the threshold of either $5 \mathrm{~cm} / \mathrm{sec}$ or $8 \mathrm{~cm} / \mathrm{sec}_{2}$. These thresholds were chosen so as to reduce false-start mistrials. Travel times were coded as the time between the liftoff time and when the pointing movement landed. Landings were logged the moment that the velocity of the motion controller dropped below either 5 or $15 \mathrm{~cm} / \mathrm{sec}_{3}$. Landing accuracy was measured as percent hit rate, which was calculated based on the logged XY location of the motion controller at landing. Euclidean distances between landing position and target were categorized as hits or misses according to

2 Experiment 1 was completed in two phases with different liftoff detection thresholds. Preliminary analyses revealed no differences in liftoff times between participants of the two phases, so they were pooled into a single sample.

${ }_{3}$ Stop thresholds were increased to $15 \mathrm{~cm} / \mathrm{sec}$ in phase two in attempt to reduce the false stops observed in phase one of the Experiment 1. 
whether there was there was any overlap between the cube and target as per the calculations in Appendix B: Data preparation

\section{Procedure}

Upon arrival, participants were briefed about the experimental protocol and the apparatus and then asked to sign a consent form (see Appendix A: Experiment documents) and encouraged to ask any questions they had. Participants put on the VR headset only once they had expressed understanding of the procedure. The potential for disorientation and or nausea during the experiment were specifically addressed. Consideration was given to the potentially uneasy state of having one's vision completely occluded by inviting participants to remove the headset if they wish to take a break and by encouraging an open dialogue at all times between participant and experimenter. The experimenter asked for permission each time it was necessary to manually adjust any element of the experiment apparatus on the participant's body (headset adjustments, controller placement in hand). Participants were informed that if they became disoriented or dizzy that their first response should be to close their eyes in order to reset the vestibular system, or to remove the headset and take a break for some water or fresh air. Participants were also informed that if they were uncomfortable at any point, they were free to leave the experiment without academic penalty. Throughout Experiment 1 and subsequent experiments there were no occasions when participants needed a break or left the study due to discomfort.

The experiment began with a practice block containing 20 trials where participants became familiar with reaching in the virtual environment with their left or right hand according to a 2-level counterbalancing between participants. Practice trials preceded the first block of the left- and right-hand experimental blocks. Practice trials were identical to the experimental trials but contained instructive text to return to the start position when the trial ended. For the 
experiment proper there were four 100-trial reaching blocks. Hand was counterbalanced and alternated across blocks. Participants were asked to place the hand not in use on their lap and asked to begin by placing their reaching hand at the start position on the desk near their body midline.

Participants were instructed to initiate reaching as soon as the target appeared and to move as quickly as possible without sacrificing reaching accuracy. To facilitate fast reaches participants were instructed to be ready to move as soon as the fixation cross appeared. Once the target had been reached it disappeared and participants were instructed to leave their hand a moment in order to ensure the capture of their stop, then to return their hand to the start position. After a random interval between 1000 and $3000 \mathrm{~ms}$ the next trial began with a fixation cross then the next target appeared after the next randomized interval. Trials proceeded in this manner until a block was completed. Following each block participants were asked to remove the headset and check in with the experimenter regarding their comfort before switching hands for the next block of trials. The experiment ran for approximately 1 hour and was followed by a short questionnaire and a post-experiment structured interview for a further 15 minutes. Participants were asked if they had any further questions and were given a debrief form (see Appendix A: Experiment documents for questionnaire and debriefing form).

\section{RESULTS}

\section{Pointing Data}

Latencies. Latency data were trimmed according to the procedure outlined in Appendix B: Data preparation. The liftoff time and travel time data were submitted to separate 2 (Hand) x 5 (Target) repeated measures ANOVAs and 95\% confidence intervals were calculated for visual analysis of the interaction (see Hollands \& Jarmasz, 2010). The omnibus ANOVA for the liftoff 
Table 1. Omnibus effects for 2 (Hand) x 5 (Target) repeated measures ANOVAs for pointing movement liftoff time and travel times measured in ms.

\begin{tabular}{llcrccc}
\hline Latency & Effect & df (adjusted) & \multicolumn{1}{c}{ MS } & $F$ & \multicolumn{1}{c}{$p$} & $\mathrm{y}_{\mathrm{p} 2}$ \\
\hline Liftoff Time & Target & $4(3.1)$ & $4223.26 \mathrm{a}$ & 26.868 & $<.001$ & .350 \\
& Error & $200(154.9)$ & 157.18 & & & \\
& Hand & 1 & 3554.31 & 8.065 & .007 & .139 \\
& Error & 50 & 440.72 & & & \\
& Hand x Target & $4(3.1)$ & $435.08 \mathrm{a}$ & 3.782 & .011 & .070 \\
& Error & $200(154.4)$ & 115.03 & & & \\
Travel Time & & & & & \\
& Target & $4(3.3)$ & $1897.24 \mathrm{a}$ & 4.582 & .003 & .084 \\
& Error & $200(166.3)$ & 414.02 & & & \\
& Hand & 1 & 69802.46 & 21.567 & $<.001$ & .301 \\
& Error & 50 & 3236.60 & & & \\
& Hand x Target & $4(2.7)$ & $27670.05 \mathrm{a}$ & 67.62 & $<.001$ & .575 \\
& Error & $200(134.2)$ & 409.20 & & & \\
\hline
\end{tabular}

${ }_{\mathrm{a}}$ Greenhouse-Geisser adjusted

time data is presented in Table 1. There were significant main effects of Target and Hand, as well as a significant interaction between these factors. The main effect of Hand showed the predicted effect in that liftoff time times were shorter for left-hand (351) than right-hand (357ms) trials. This replicates the findings from Elliott et al. (1993) and Boulinguez et al. (2001a, 2001b) and suggests that the left and right cube effector in the virtual environment are being represented in a manner similar to natural left and right hands. As shown in Figure 5, the main effect of Target followed a quartic trend, where liftoff times were shortest to Target 2 (351ms) and Target 4 (349ms) as compared to Targets 1 (354ms), Target 3 (363ms), and Target 5 (352ms). There was an interaction between Hand and Target wherein there was a greater difference between the liftoff time of the hands for Targets 1 and 2 (left of centre) as compared to the rest of the targets.

The omnibus ANOVA for the travel time data is reported in Table 1. As predicted, there was a significant main effect of Hand where, on average, travel times to targets were faster on right-hand $(478 \mathrm{~ms})$ than left-hand $(501 \mathrm{~ms})$ trials. There was also a main effect of Target where travel times were shorter to Target 1 (left-most target, $487 \mathrm{~ms}$ ), Target 3 (central target $488 \mathrm{~ms}$ ), 


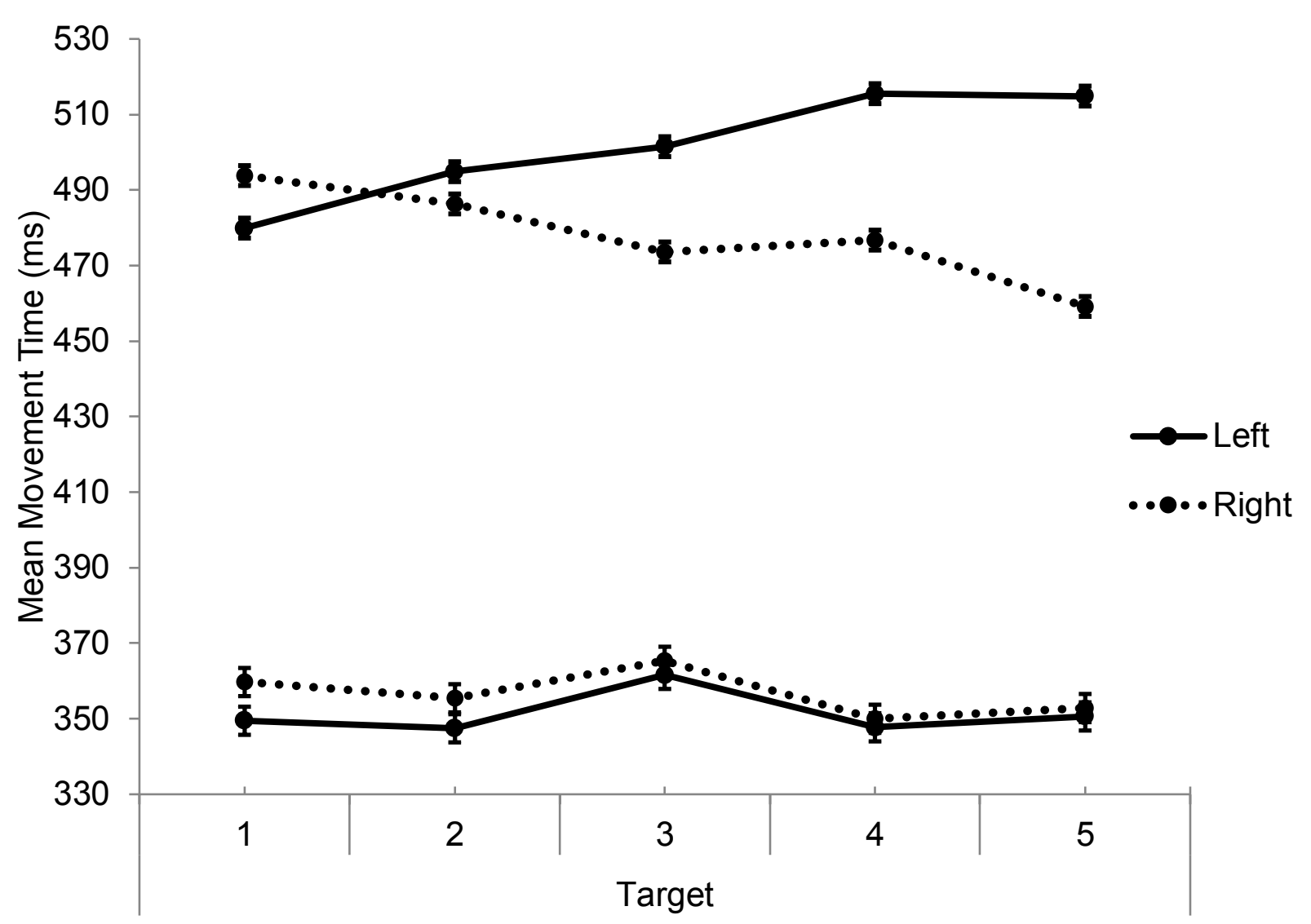

Figure 5. Mean travel times (top) and liftoff time (bottom) in ms for the left and right hands at Targets 1-5. Error bars represent 95\% confidence intervals for the comparison of Hand as per Hollands \& Jarmasz, 2010.

and Target 5 (right-most target, 487ms) than to Target 2 (left of centre, $491 \mathrm{~ms}$ ) and Target 4 (right of centre, $496 \mathrm{~ms}$ ). The interaction between Hand and Target was also significant. As shown in Figure 5, left-hand movements were faster to leftward targets (Targets 1 and 2) than to rightward targets (Targets 4 and 5). Conversely, right-hand movements were faster toward rightward targets were faster to leftward targets than to rightward targets. This crossover interaction is consistent with the prediction that each hand would travel faster to same-side targets as was found by Fisk \& Goodale (1985). 
Goodale (1985) and replicated by Boulinguez et al. (2001a; 2001b) and corroborates the evidence from the liftoff times that the virtual left and right cube are represented in a manner similar to the natural left and right hands.

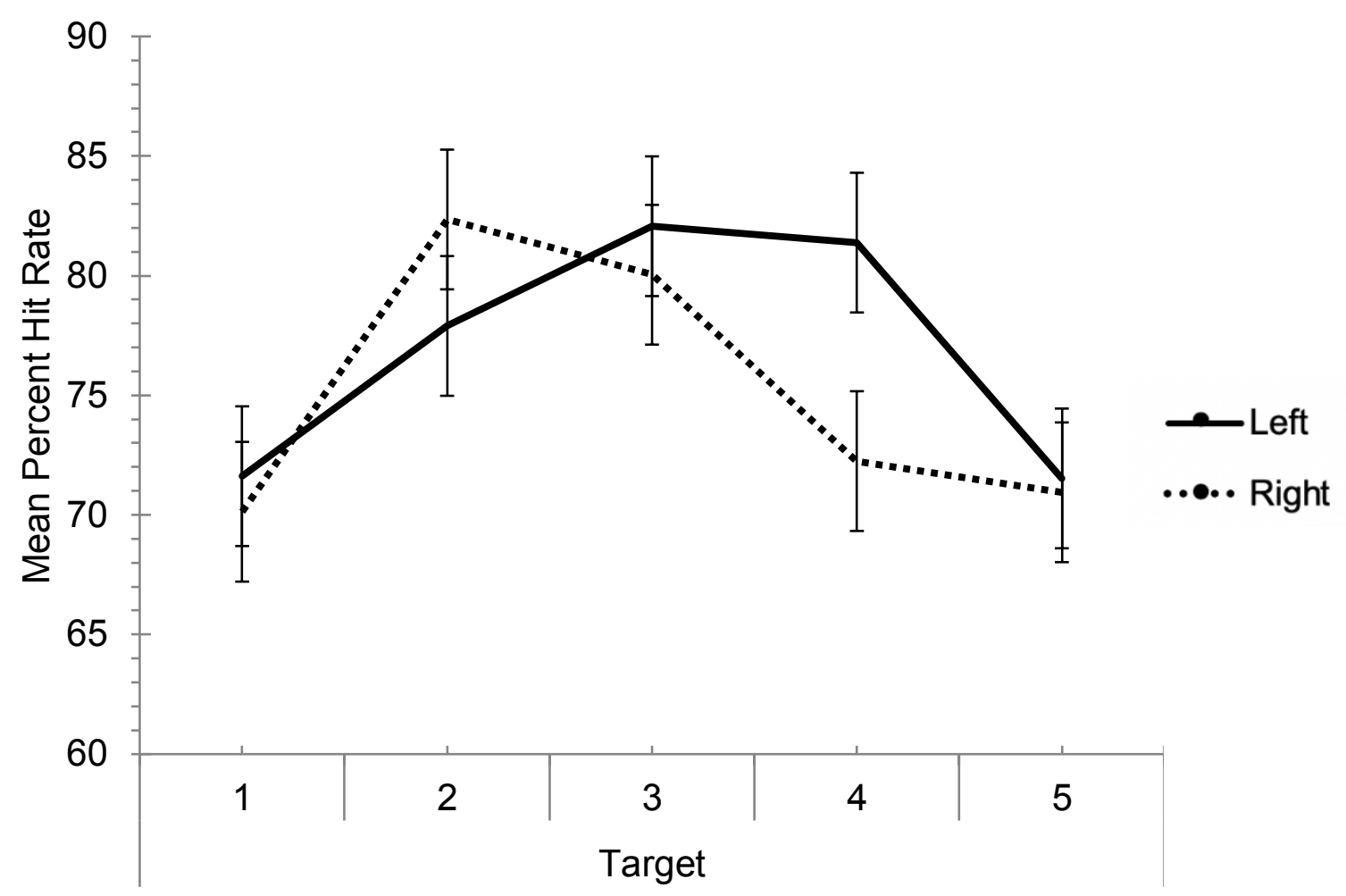

Figure 6. Mean Percent Hit Rate to all targets by Left-hand (solid) vs. Righthand (dashed). Error bars represent $95 \%$ CIs for the comparison at Hand as per Hollands and Jarmasz, (2010).

Accuracy. To examine reaching accuracy, each landing position was converted into Euclidean distance from a target. The final position of the motion controller on each trial was subtracted from the position of the target on the trial. This procedure resulted in negative values for landing positions that were short and left of the targets, and positive values for landing positions that were beyond and right of the targets in the depth and lateral dimensions, respectively. Euclidean distances were then used to determine whether the final position of the controller was an accurate landing (i.e., on or touching the target) or an error (i.e., not touching 
Table 2. Omnibus effects for 2 (Hand) x 5 (Target) repeated measures ANOVA for landing accuracy calculated as percent hit rate per hand at each target.

\begin{tabular}{lcrrrl}
\hline Effect & df (adjusted) & \multicolumn{1}{c}{ MS } & $F$ & $p$ & yp2 \\
\hline Target & $4(2.9)$ & $3285.85 \mathrm{a}$ & 20.281 & $<.001$ & .285 \\
$\quad$ Error & $200(148.8)$ & 162.02 & & & \\
Hand & 1 & 400.53 & 0.741 & .393 & .014 \\
$\quad$ Error & 50 & 540.57 & & & \\
Target x Hand & $4(2.7)$ & $896.79 \mathrm{a}$ & 4.497 & .002 & .081 \\
$\quad$ Error & $200(139.7)$ & 199.43 & & & \\
\hline
\end{tabular}

aGreenhouse-Geisser adjusted

the target at all; see Appendix B: Data preparation for calculations). Accuracy was converted to percent hit rates.

Percent hit rates were analyzed in a 2 (Hand) x 5 (Target) repeated measures ANOVA (see Table 2). Confidence intervals (95\%) were used to examine the interaction (see Figure 6). There was no main effect of Hand, but there was a significant interaction between Target and Hand. As shown in Figure 7, there was no difference between left- and right-hand hit rates apart from target 4, where left-hand hit rates were higher than right-hand hit rates. The lack of significant difference between the left- and right-hand trials is not consistent with predictions but means that the interpretation of the latency data remains uncompromised. There was a significant main effect of Target. As shown in Figure 6, hit rates were highest for Target 3 $(81 \%)$ and decreased for targets outward to the left (Target 1, 71\%; Target 2, 80\%) and right (Target 4, 77\%; Target 5, 71\%).

\section{Questionnaire Data}

After the experiment participants completed a short questionnaire adapted from Botvinick and Cohen (2002). The questionnaire was used to index participants' subjective experience of embodied presence by asking them to rate their agreement with statements about the nature of the relationship between their own hand and the virtual cube. Participants were instructed to 


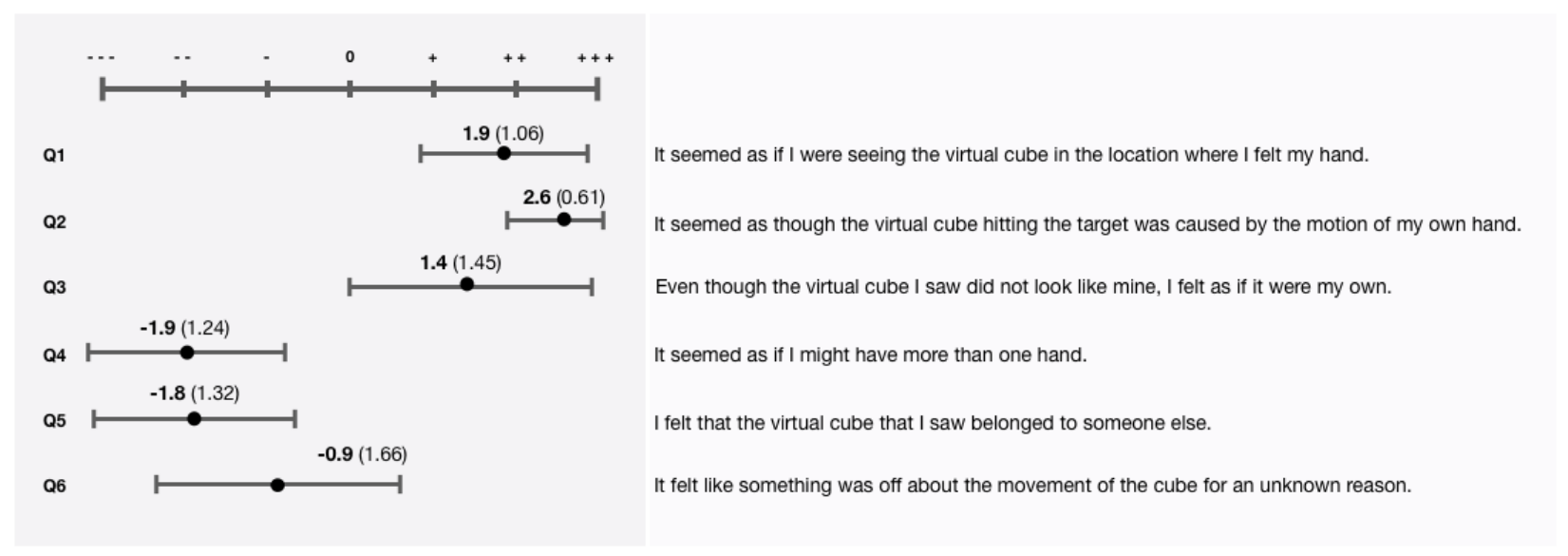

Figure 7. Mean responses to post experiment questionnaire where "- - -" signified strong disagreement and " +++ " signified strong agreement and error bars represent standard deviation of the item mean

indicate their choice by circling one of the vertical lines ranging from negative to positive ratings for each statement. These indications were converted to numbers ranging from -3 to -1 for the negative symbols on the left, 0 for the neutral position, and from 1 to 3 for the positive positions on the right. Single-sample t-tests revealed that responses for each item were significantly different from neutral ( $\mathrm{ps}<.05$ after Bonferroni adjustment for multiple comparisons4). Items describing a strong relationship between participants' movements (items 1-3) and those of the cube (items 4-6) were generally met with agreement while catch items (Items 4 and 5) were not (see Error! Reference source not found.). This pattern of responses suggests that participants experienced embodied presence in the virtual pointing task.

\section{DISCUSSION}

\section{Pointing kinematics in VR reflect natural pointing behaviour}

Differences between the left and right hands on liftoff times were consistent with predictions: left-hand pointing movements were initiated more quickly than right-hand pointing movements. This is consistent with findings from Elliott et al. (1993) and Boulinguez et al. 
(2001), and supports the assumption that the left hand receives motor commands faster than the right hand due to the left hand's connection to the right-hemisphere - which has superior processing speed and spatial reasoning capacity as compared to the left-hemisphere (Bryden, 1982). Importantly, this pattern of findings suggests that the brain represents virtual left- and right-hand cube effectors in the same manner as hands in the physical world. Similar support for this claim lies in the difference in travel time between the left- and right-hand pointing movements, where the right-hand indeed traveled to targets more quickly than the left-hand. Also consistent with predictions was the finding that each hand traveled more quickly to targets in its own hemispace (see Figure 5). This aligns with the findings of Fisk and Goodale (1985) and replications by Boulinguez et al. (2001a; 2001b).

There was no difference in landing accuracy between left- and right-hand pointing movements.. Examination of Figure 5 shows that liftoff times were fastest for right-hand pointing at Target 4 , and travel times to Target 4 were among the fastest for right-hand pointing movements as well. It is worth noting that Target 4 was aligned almost perfectly with the natural landing position of a participant's right-hand when merely extending the elbow of their right arm. It is possible that the ease with which the proximity of Target 4 could be reached caused participants to under-assign resources to monitor the accuracy of landing positions to this target location. Indeed, Figure 6 demonstrates the uniqueness of right-hand pointing accuracy to Target 4; the mean hit rate to Target 4 is well below that of adjacent target locations. Due to the size of the virtual cube, certain landing positions within $2 \mathrm{~cm}$ of a target might have caused a visual occlusion that appeared as an accurate landing. This may have artificially inflated participants' assessment of their pointing accuracy.

\section{Embodying the virtual cube}


Responses to the questionnaire revealed that participants strongly agreed that the motion of the cube was directly under their control. Participants also agreed, although less strongly, with the statement that the cube's location was an accurate representation of their hand's location. It would seem then, that despite some spatial or temporal inconsistencies, participants felt that the cube landed where their own hand landed. Participants also agreed with the statement that the cube felt like their hand, even though it did not look like their hand.

In sum, participants learned to use the cube as a pointing tool and felt to some extent that it represented their own hand. Taken with the observation that left- and right-hand pointing differences persist in VR, responses to the questionnaire indicate that the hands are being represented as part of the body even when they appear as a virtual cube. A potential mechanism for adoption of the virtual cube as part of the body might be the connection between the visual trajectory of the virtual cube and the proprioceptive information participants received from the movement of their own hand and arm.

Proprioceptive Contributions to Owning Movement. In a typical setting, a person rarely questions the origin of their movements or considers the intricate interaction of sensorimotor feedback from which motor control arises. It is only in situations of impoverished sensory feedback or unexpected action outcomes, such as those experienced in a virtual environment, that there may be a need to inspect the relationship between intention and action. Studies of neurological patients have shown that when information about the body's movement or position is not intact, a full mental representation of how we might use our body to interact with the environment becomes compromised. This representation of how the body might interact with the environment is presently considered as embodied presence. Studies of body representation in natural settings illustrate why it is important that participants reported that the 
cube followed their own movements: feeling a sense of ownership over the movements of the cube in the virtual environment may be the mechanism that allows effector factors to support embodied presence.

Balslev, Cole and Miall (2008) demonstrated that proprioception is fundamental to a sense of ownership over one's actions. Patient I.W. incurred neurological damage such that his brain did not receive somatosensory inputs from light touch stimuli or proprioceptive signals, whereas other inputs including muscle activity were received intact (pathology described by Cole \& Sedgwick, 1992; cited in Balslev et al. 2008). This damage meant that I.W. was unable to learn new motor tasks without visual feedback. A group of healthy controls participated in movement ownership and discrimination control tasks and were compared to I.W. During the movement ownership task participants were required to move their finger to either the top or bottom of a trackpad according to a visual cue. Participants' hands were visually occluded while they viewed a screen displaying their putative cursor movements translated to the horizontal axis. On some trials the cursor appeared to move in time with participant's finger movements, but on other trials the cursor moved slightly before or slightly after participants' finger movements. Verbal responses were given on each trial as to whether it felt like the cursor movement was caused by participants' finger movement, or some other factor. Patient I.W. was significantly less accurate than healthy controls in deciding whether his finger movements caused the cursor movements. In a motion discrimination task, however, I.W. was able to accurately report whether a mouse cursor had moved leftward or rightward. From this Balslev et al. concluded that I.W.'s poor performance in the movement ownership task was not an artifact of increased attentional demands for motion processing. Rather, the absence of visual feedback of I.W.'s 
finger movements paired with the usual absence of proprioceptive input to his brain impaired his ability to ascribe ownership to cursor movements that he had in fact caused.

As demonstrated with patient I.W., proprioception plays an important role in the ownership of movements, especially in case of impoverished visual feedback. This means that proprioception may play a more important role in virtual environments than natural environments because VR often presents participants with visual representations of themselves that is lower fidelity and difficult to render in real-time. Indeed, Experiment 1 demonstrates that despite impoverished visual feedback, participants still claimed they made the movements they saw in the virtual environment.

During the post-experiment interview participants were asked whether they felt, at any point, that their reaching arm became invisible. Most participants claimed they had not experienced or even considered this sensation because they, as one participant coined, "could feel [their] arm the whole time". This suggests that when reflecting on their experience in the virtual pointing task, participants relied at least partially on the proprioceptive information about their arm's actual location and movement to construct a representation of their body in VR. Statements in the family of 'it just felt like it was my hand' evoke a similar sense as classic definitions of presence as the 'feeling of being there'.

\section{Summary}

Experiment 1 established the VR pointing task that was used throughout this thesis. The pointing task was modelled after tasks used in the motor learning literature in which it has been found that left-hand pointing movements are initiated more quickly than right-hand pointing movements. Experiment 1 demonstrated the same asymmetries between the left and right hands 
for a virtual pointing task in which the participants' hands were represented as a virtual cube. The results suggest that the virtual cube effector was embodied to some degree. 


\section{EXPERIMENT 2: FIRE IN THE VIRTUAL ENVIRONMENT}

Experiment 2 examined how changes in a virtual environment affect embodied presence. This was done by introducing a wall of virtual fire into the environment that was used in Experiment 1. Two locations were chosen for the virtual fire: a location behind the targets (far from participants) and a location between the targets and start position (near participants). The virtual fire appeared just before participants initiated their reach toward targets. It was hypothesized that if participants experienced a strong sense of embodied presence then they should exhibit differences to their pointing movements on trials where fire is present in the virtual environment. A model of the processes involved in coordinating motor control was used to make specific predictions about the effects of virtual fire on pointing movements.

Motor planning is a broad term that describes a series of processes involved in detecting stimuli, making plans for task completion, and coordinating a set of motor commands necessary for the execution of a movement. These task-oriented movements are referred to as motor goals, which are informed by task requirements and serve as a set of instructions to pass downstream to the motor planning system (see Wong, Haith, \& Krakauer, 2015 for a review). This process of requirements gathering and subsequent movement planning has been broken into a "what" and "how" phase, respectively. Once motor plans have been set, there are different theories of how movement is coordinated. Theories of motor control differ in their description of whether movements are informed and adjusted by incoming information (i.e., closed loop models, Adams, 1971) or whether they rely on pre-programed instructions and then adjust based on outcome (i.e., motor programs, Keele, 1968). Modern models of motor control are based on a hybridized view, where the motor system takes advantage of both fast, pre-coordinated execution 


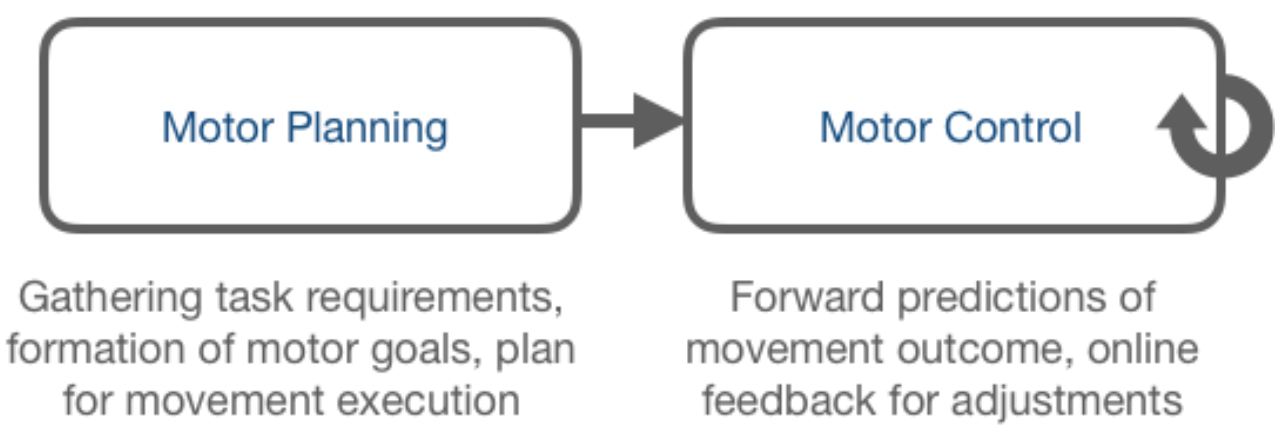

Figure 8. Simplified model of relationship between motor planning and motor control.

(i.e., a forward feed-forward loop) as well as controlled, online sensory information (i.e., a feedback loop). Together, motor planning and motor control describe the two major phases of how coordinated movement is carried out by the brain and body (see Figure 8). These two phases will serve as a model for examining the influence of environment on pointing movements.

Because the virtual fire in Experiment 2 is being added in synchrony with the appearance of targets, it is expected that fire should be factored into the motor planning phase, that is, the formation of the motor goal and how it should be carried out. It is therefore predicted that the influence of virtual fire on the motor planning stage will be observed as alterations to the liftoff time for pointing movements. Further, if the effect of fire is one of realistic salience rather than a novelty effect then there should be a difference between the locations of fire in that fire located near participants should have a greater effect than fire far from participants. It is not likely that virtual fire would affect the motor control stages of movement because this stage represents the monitoring phases wherein movements are compared to the motor goal (i.e., hitting the target). Ongoing movement monitoring should remain relatively unaffected by the presence of virtual fire. Accordingly, the virtual fire should not affect travel time to targets.

For Experiment 2 the visual fidelity of the virtual effector was increased by replacing the virtual cube with a full fidelity hand model (see Figure 9). Presenting participants with a more realistic looking representation of their hands may increase their experience embodied presence 
compared to when they use a virtual cube effector for the pointing task. Finally, because the focus was on the environmental factor and not effector, only right-hand pointing trials were included in Experiment 2.

\section{METHODS}

\section{Participants}

Participants were 45 undergraduate students at Carleton who participated for $1 \%$ bonus credit toward an eligible course. As in Experiment 1, participants were asked about their experience with the VR technology but were not excluded for having prior exposure. Participants selected to be right-handed, with normal or corrected-to-normal vision and with a neurological history free from any motor control difficulties or conditions predisposing them to nausea (see Appendix A: Experiment documents). Data from two participants was removed due to technical failure during the experiment, leaving a total of forty-three participants.

\section{Apparatus}

Experiment 2 utilized an Oculus Rift Consumer Version 1 (CV1) VR headset with improved visual resolution $(2160 \times 1200)$ and frame rate $(90 \mathrm{~Hz})$ compared to the Oculus Rift VR headset used in Experiment 1. This technical upgrade did not alter the operational procedures of using the head-mounted display outlined in Experiment 1.

In addition to an updated head-mounted VR display, Experiment 2 utilized a Leap Motion tracking system (Ultrahaptics, Ltd) which enabled hand tracking and a high-fidelity hand representation. The Leap Motion tracker was mounted to the front of the Oculus Rift headset and 


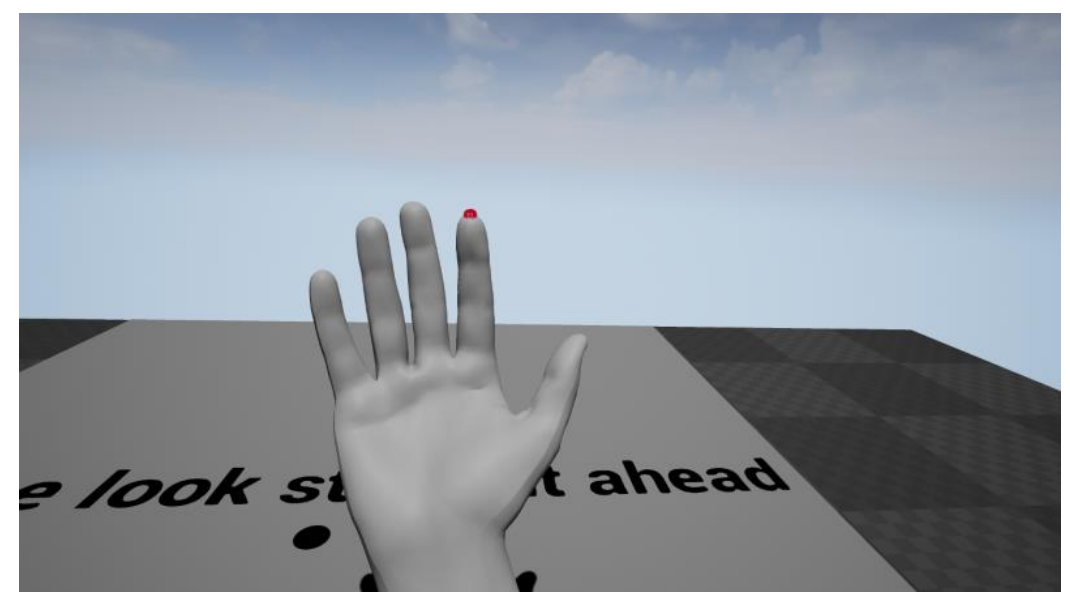

Figure 9. High fidelity representation of the hand as well as the collision volume (red dot) attached to the index finger (not visible during the experiment).

uses two infrared cameras to capture hand and finger motion. Participants' hands were tracked with full finger fidelity and appeared in VR as mannequin-like hands (see Figure 9). The addition of the Leap Motion controller allowed for a new condition in Experiment 2 where participants were able to maneuver high-fidelity hands for the pointing task. Some adjustments were made to the stock tracking algorithms to stabilize the virtual hand movement (see Appendix E: Technical Specifications for details).

To determine accurate landings collision volumes were defined in the virtual environment. Collision volumes are volumes of space within which certain actions trigger certain outcomes. One such collision volume was defined as a cylinder placed on each target with a width spanning the target $(1.5 \mathrm{~cm}$ radius) and a height of $3 \mathrm{~cm}$ below or above the target to account for small discrepancies in the detected and presented vertical location of participants' fingertips. Another collision volume was defined as a small sphere attached to the end of the pointer finger of the right hand (see Figure 9). When the participant ended their reaching motion and these two collision volumes were in contact, the program signaled an accurate landing and 
played an auditory chime. The same collision mechanism was utilized at the start position to allow participants to trigger the start of each trial by "pressing" the start button.

\section{Display and Stimuli}

The virtual environment and reaching task remained the same as that described in Experiment 1 with two additions; an auditory chime was added to signal accurate landings to participants and virtual fire was added to the reaching environment. A virtual wall of fire was presented at a location either behind or in front of the target locations. This meant that fire was presented both near to and far from the participant at 20 and $40 \mathrm{~cm}$, respectively. The onset of the fire was triggered simultaneously with the appearance of the targets but the length of the lifecycle of the fire asset meant that it reached peak intensity approximately $350 \mathrm{~ms}$ after target onset. This meant that the wall of fire appeared most prominently after participants had already initiated their reach (see Figure 10).

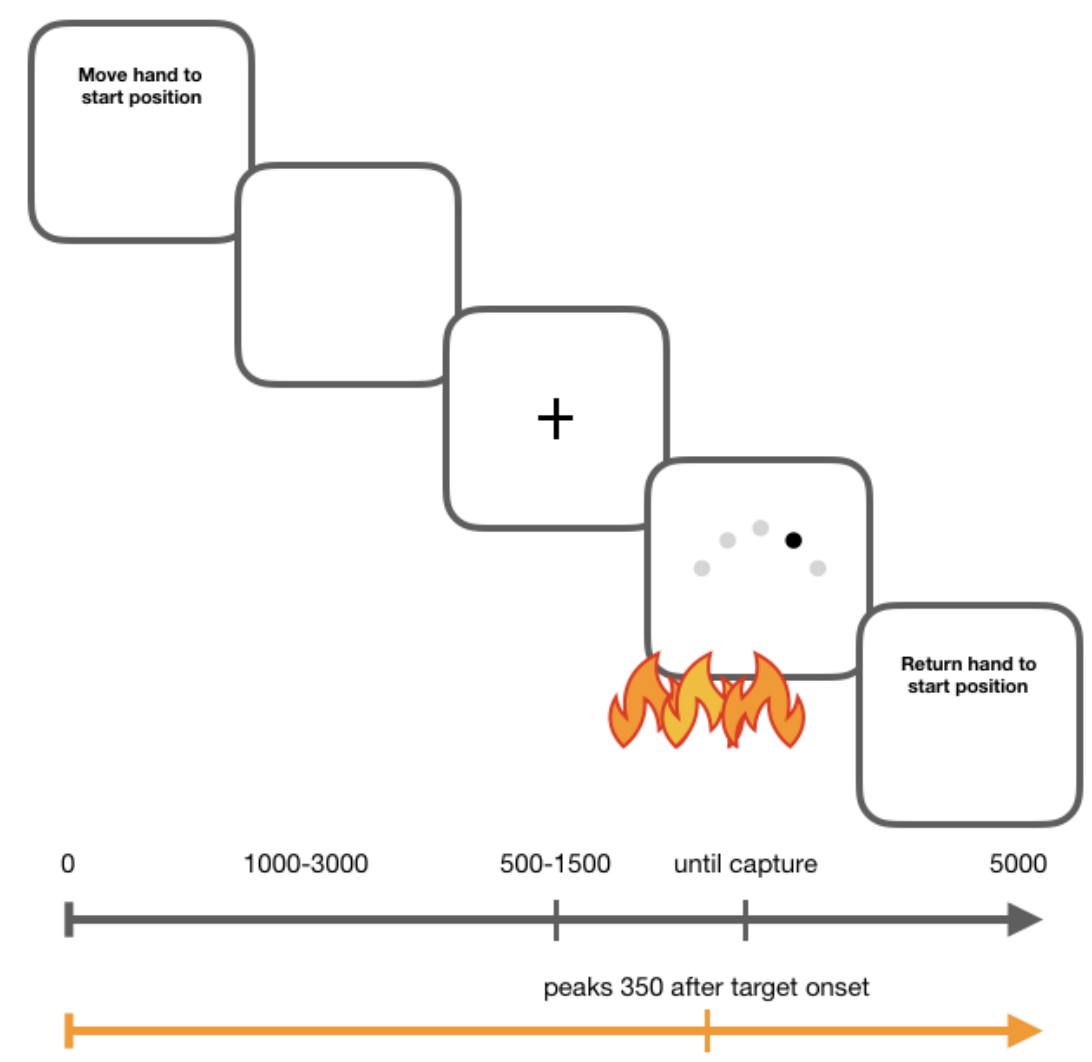

Figure 10. Trial sequence diagram, time intervals measured in ms. 
In order to rule out the possibility of a startle effect, virtual fire was presented at two locations on different trials: a location between the start position (near the body) and the target space, and a location behind the target space. This resulted in both a near fire and a far fire condition of the environment factor, on near fire trials participants had to make their pointing movements through the wall of fire to capture the target. If the influence of virtual fire on participants' pointing behaviours was due to a startle or a novelty effect, then there should be no difference in the influence of fire on pointing behaviours between the two locations of fire. If, however, the effects observed in Experiment 2 are due to participants' experience of embodied presence in VR changes to pointing behaviours should be more dramatic in the near fire than the far fire condition, where the fire poses no immediate threat to participants' adopted virtual effector.

\section{Design}

Experiment 2 utilized a 3 (Fire: near, far, none) x 5 (Target) repeated measures design.

\section{Procedure}

Participant briefing and consent protocol were the same as that described in Experiment 1 with the additional mention of the inclusion of virtual fire in the reaching task (see Appendix A: Experiment documents). Setting up Experiment 2 was similar to that of Experiment 1 with the additional task of calibrating the height of the virtual table to account for small differences in how the Leap Motion controller calculated participants' hand locations. These differences in hand position could result in the hand or cube appearing slightly above or below the table even when the physical hand was resting on the table. Calibration was achieved by asking participants to start each block by resting their pointing finger on a test target while the experimenter adjusted the virtually apparent height of the table. 
One critical difference from Experiment 1 was that the new set of instructions for Experiment 2 involved asking participants to adopt a pointing gesture as though they were making the letter " $L$ " with the thumb and forefinger. The rest of the fingers were curled under the palm and participants were instructed to maintain this posture of the hand while driving their reaches with movements of the arm and shoulder. The practice phase of each block was used to ensure that the Leap Motion controller was detecting participants' landings accurately and that the auditory chime was detected by participants when they landed within the defined target collision volume. The experiment was paused halfway through the experimental trials so as to provide a break for participants when the experimenter checked-in to verify that participants were comfortable to continue.

The experiment began with 60 practice trials. The presentation of fire was restricted to the 400 experimental trials randomized at the trial level per each 200-trial half of the experiment. Fire was presented with a probability of .30 for a total of 60 fire-trials in each half of the experiment. At the end of the experiment participants completed the same adapted Botvinick and Cohen (2002) Questionnaire from Experiment 1 with wording adjustments made to reflect the use of a virtual hand instead of cube in Experiment 2. The experiment ran for approximately 30 minutes and participants were giving a short interview about their experience and then debriefed according to the same procedure as described for Experiment 1.

\section{RESULTS}

\section{Pointing Data}

Latencies. Pointing behavioural data were trimmed in the same manner as Experiment 1 (see Appendix B: Data preparation) resulting in $10.2 \%$ data loss. The liftoff time and travel time 
Table 3. Omnibus effects on liftoff times in the 3 (Fire Location) x 5 (Target) repeated measures ANOVA.

\begin{tabular}{lcrccc}
\hline Effect & df (adjusted) & \multicolumn{1}{c}{ MS } & $F$ & $p$ & $\eta_{\mathrm{p} 2}$ \\
\hline Target & $4(3.1)$ & $13271.78 \mathrm{a}$ & 12.09 & $<.001$ & .224 \\
$\quad$ Error & $168(131.0)$ & $1097.75 \mathrm{a}$ & & & \\
Fire & $2(1.5)$ & $110960.88 \mathrm{a}$ & 44.542 & $<.001$ & .515 \\
$\quad$ Error & $84(63.6)$ & $2491.15 \mathrm{a}$ & & & \\
Target x Fire & $8(5.6)$ & $1145.57 \mathrm{a}$ & 1.509 & .153 & .035 \\
$\quad$ Error & $336235.9)$ & $759.11 \mathrm{a}$ & & & \\
\hline
\end{tabular}

aGreenhouse-Geisser adjusted

data were submitted to separate 3 (Fire: none, near, far) x 5 (Target) repeated-measures

ANOVAs.

The omnibus test for liftoff time data is presented Table 3. Of primary interest was a significant main effect of Fire where liftoff times were different depending on fire location. Examination of the error bars on Figure 11 show the simple effect of Fire at the different levels of Target, where liftoff times were shortest when there was no fire in the virtual environment (458ms), and delayed when there was fire far from participants (482ms) and delayed more dramatically when fire was near participants (497ms). The reliable difference in liftoff times

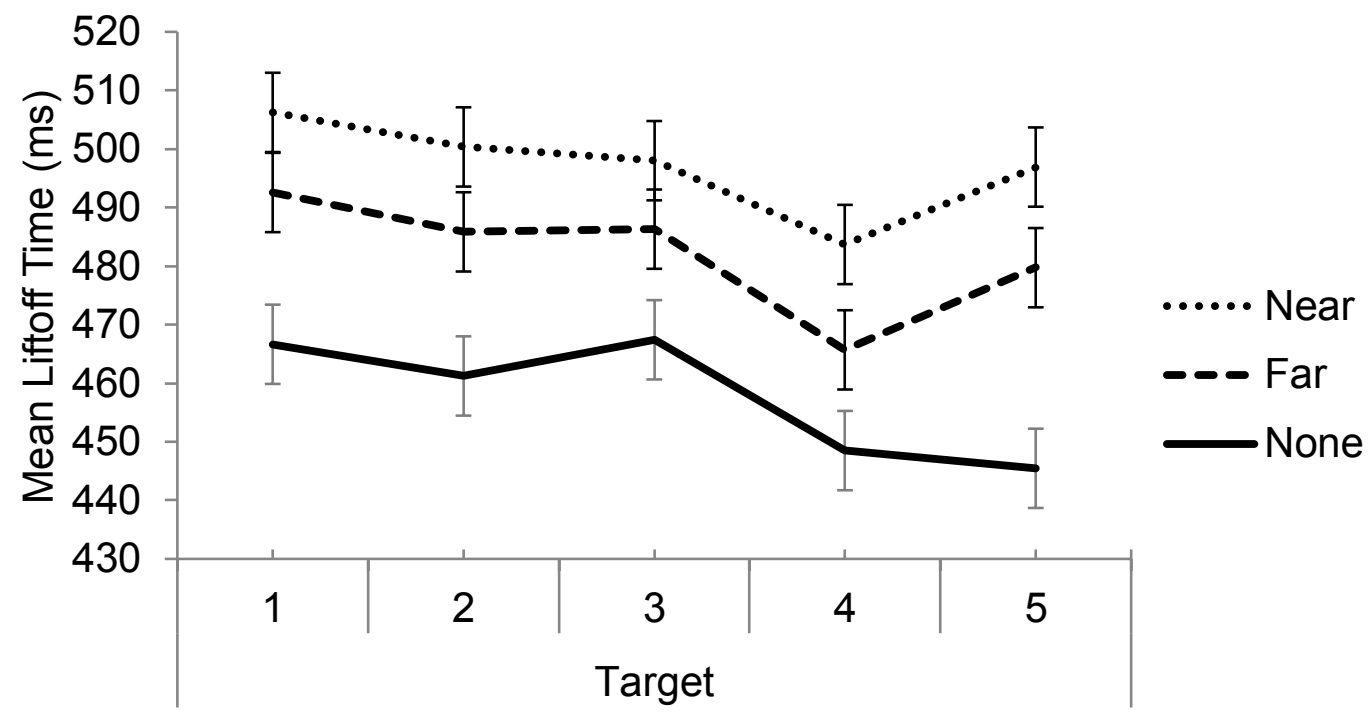

Figure 11. Mean liftoff times to all targets for the None, Near, and Far conditions of Fire. Error bars represent 95\% CIs for the comparison at Fire as per Hollands \& Jarmasz, 2010. 
Table 4. Omnibus effects on travel times in the 3 (Fire Location) x 5 (Target) repeated measures ANOVA.

\begin{tabular}{lcrccc}
\hline Effect & df (adjusted) & \multicolumn{1}{c}{ MS } & $F$ & $p$ & $\eta_{\mathrm{p} 2}$ \\
\hline Target & $4(1.8)$ & $145375.28 \mathrm{a}$ & 65.091 & $<.001$ & .608 \\
$\quad$ Error & $168(75.3)$ & $2233.41 \mathrm{a}$ & & & \\
Fire & $2(1.6)$ & $345.89 \mathrm{a}$ & 0.54 & .542 & .013 \\
$\quad$ Error & $84(65.5)$ & $640.39 \mathrm{a}$ & & & \\
Target x Fire & $8(6.6)$ & 230.31 & 0.896 & .520 & .021 \\
$\quad$ Error & $336(267.8)$ & 256.97 & & & \\
\hline
\end{tabular}

aGreenhouse-Geisser adjusted

between the near and far locations of the fire rules out the possibility that the mere presence of fire alters pointing movements (i.e., it is not a novelty effect). There was also a significant main effect of Target, where liftoff times were fastest at the rightward targets (Targets 4, 466ms; and Target 5, 474ms) than they were for the central (Target 3,484ms) and leftward targets (Target 1 , 489ms; and Target 2, 483ms). There was no interaction between Target location and Fire.

The omnibus results of the repeated measures ANOVA for travel times are shown in Table 4. For travel time, there was a main effect of Target where movements were faster to rightward targets (Target 4, 466ms; and Target 5, 442ms) than to the central target (Target 3,

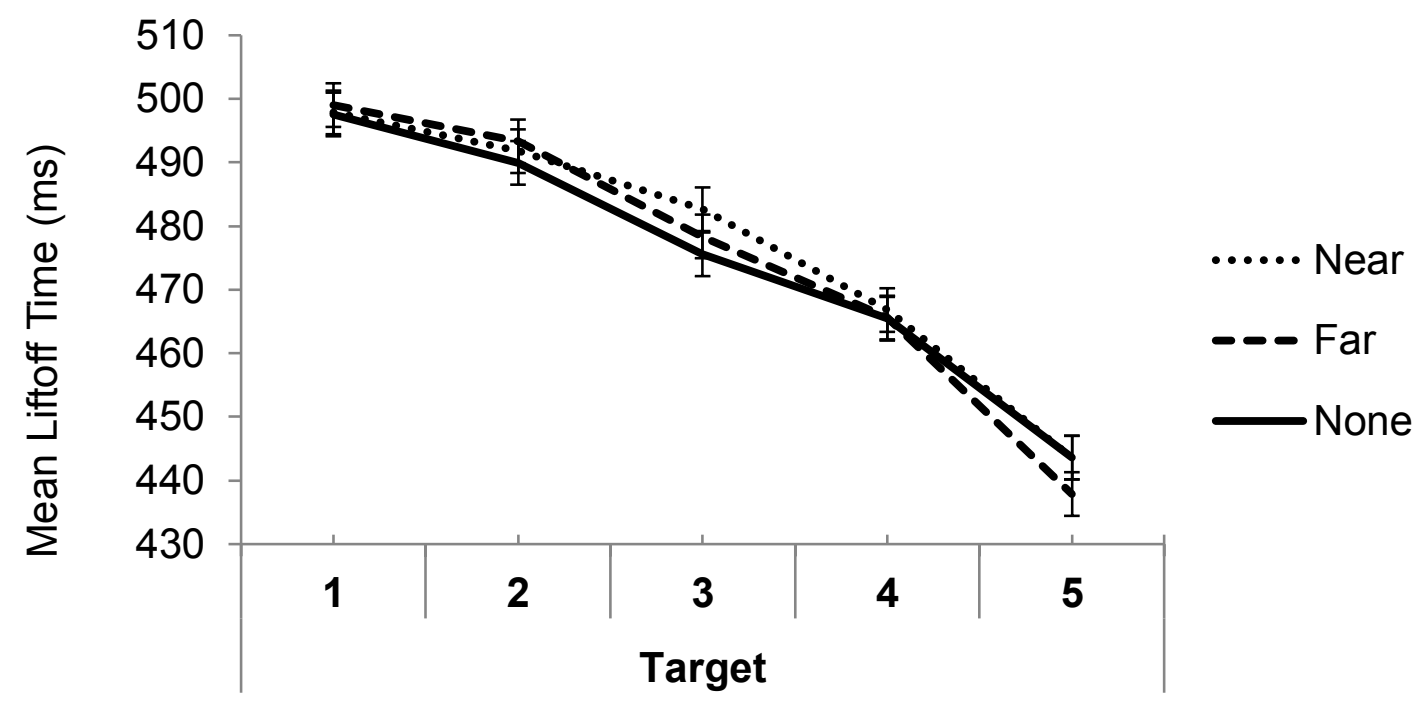

Figure 12. Mean travel times to all targets by Fire Location. Error bars represent $95 \%$ Cis for the comparison at Fire as per Hollands and Jarmasz, 2010. 
Table 5. Omnibus effects on Percent hit rate for the 3 (Fire Location) x 5 (Target) repeated measures ANOVA.

\begin{tabular}{lccccc}
\hline Effect & df (adjusted) & MS & $F$ & $p$ & $\mathrm{y}_{\mathrm{p} 2}$ \\
\hline Target & $4(2.4)$ & $175.99 \mathrm{a}$ & 175.999 & .501 & .017 \\
$\quad$ Error & $168(103.6)$ & $236.86 \mathrm{a}$ & & & \\
Fire & $2(1.6)$ & $168.74 \mathrm{a}$ & 1.234 & .291 & .028 \\
$\quad$ Error & $84(69.5)$ & $136.76 \mathrm{a}$ & & & \\
Target x Fire & 8 & 114.59 & 1.555 & .176 & .035 \\
$\quad$ Error & 336 & 73.709 & & & \\
\hline
\end{tabular}

aGreenhouse-Geisser adjusted

$479 \mathrm{~ms}$ ) and the leftward targets (Target 1, 498ms; and Target 2, 492ms; see Figure 12). This replicates the findings of Experiment 1 where right-hand pointing movements were faster to rightward than leftward targets. There were no other significant effects on travel times.

Accuracy. Pointing accuracy was examined using the same percent hit rate measure described in Experiment 1. Mean percent hit rate scores were submitted to a 3 (Fire: none, near,

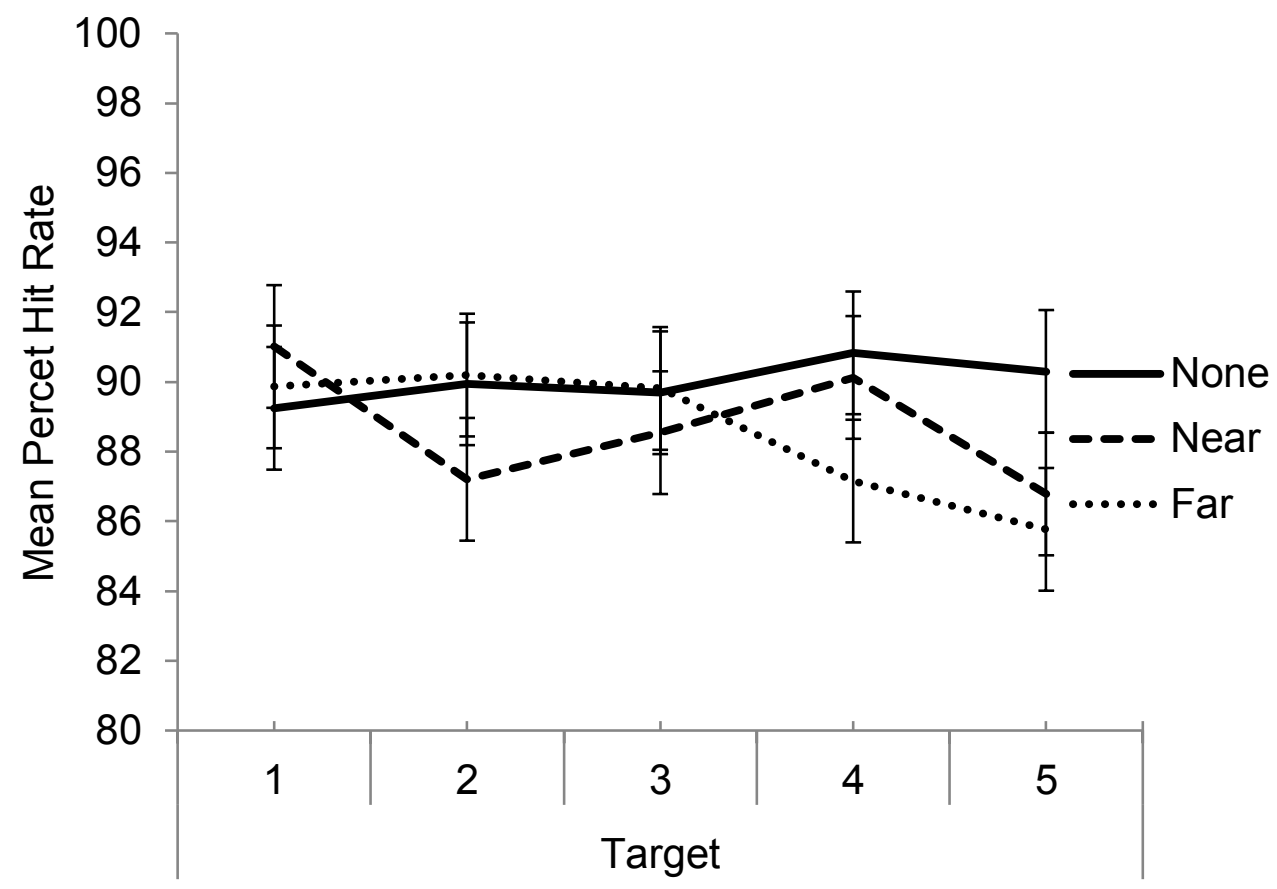

Figure 13. Mean Percent Hit Rate to all targets for the None, Near, and Far conditions of the Fire. Error bars represent 95\% CIs for the comparison at Target as per Hollands \& Jarmasz, 2010). 
far) $x$ 5(Target) repeated measures ANOVA. As shown in Table 5 there were no significant effects on landing accuracy. Mean percent hit rates are shown in Figure 13.

\section{Questionnaire Data}

After the experiment participants completed a similar questionnaire to Experiment 1 that was adapted for Experiment 2. The median response for each item is displayed in Figure 14. Independent t-tests 5 revealed that participants' responses were significantly different from neutral (ps $<.001$, with Bonferroni adjustment for multiple comparisons) on all items apart from Question 6,. As Figure 14 shows, participants agreed with items that described a good connection between their hand movements and virtual cube or hand, and generally disagreed with items that indicated a disconnection in their experience of moving the virtual cube or hand. Responses are consistent with those from Experiment 1 and indicate that participants experienced presence.

\section{DISCUSSION}

\section{Fire affects movement planning but not execution}

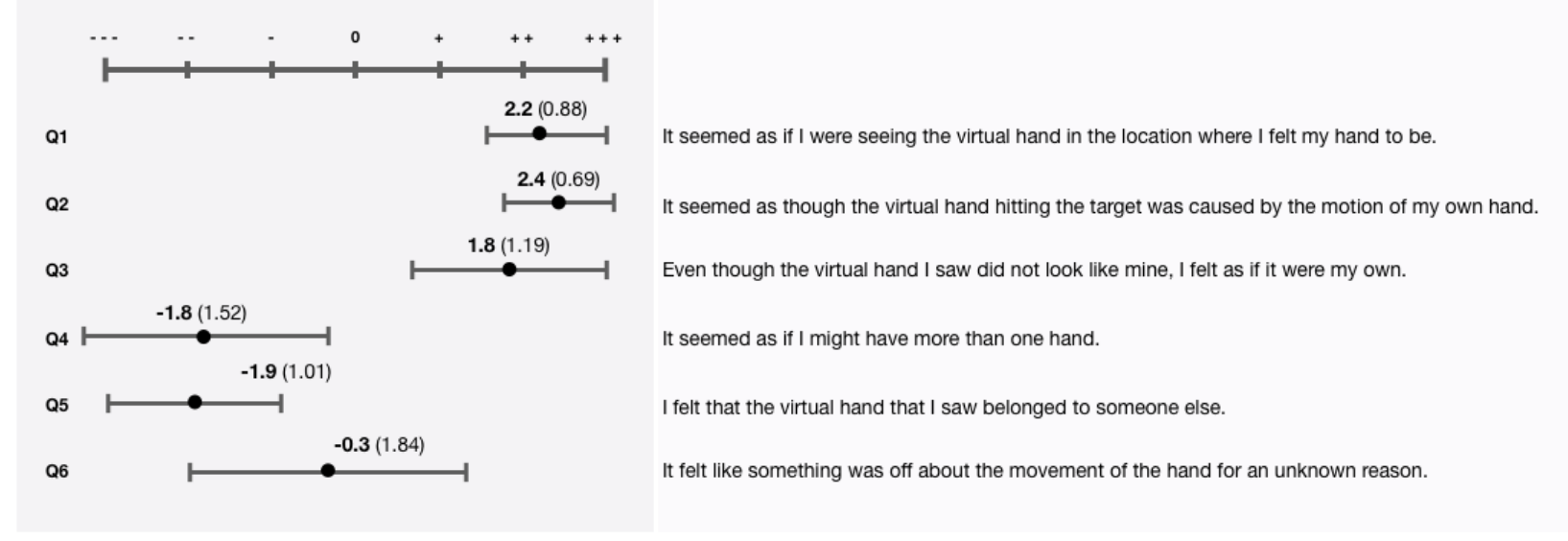

Figure 14. Mean responses to post experiment questionnaire where "- - -“" signified strong disagreement and " +++ " signified strong agreement and error bars represent standard deviation of the item mean.

5 A non-parametric alternative Wilcoxon Signed Rank test against a median of 0 was also conducted, findings were the same for all questionnaire items. 
The purpose of Experiment 2 was to examine how embodied presence is affected by factors in the virtual environment. To this end, a wall of virtual fire was introduced to the environment on some trials such that participants either had to point toward a wall of fire to hit the target (near condition) or move through a wall of fire to hit the target (far condition). Liftoff times were influenced by the presence of fire in the virtual environment: liftoffs were longer in the presence of fire than in its absence. Liftoff times were also found to be longer in the near fire than the far fire condition which confirms that the influence of fire is more than a mere novelty (startle) effect. Instead the results suggest that participants took longer to plan and initiate a movement in the presence of the virtual fire as though it may have realistic consequences for their pointing movements. This finding suggest that participants experienced a sense of embodied presence. Responses to the post experiment Questionnaire corroborate this claim: participants generally agreed with items that described a strong relationship between the virtual hands and their own hands despite the difference in appearance from their own hand.

Whereas the presence of virtual fire influenced liftoff times, fire had no impact on travel times to targets. This suggests that although changes to the environment may affect motor planning, environment factors do not affect motor execution. As discussed below, this has implications for the role of environmental factors on supporting a sense of embodied presence.

\section{Implications for embodied presence from Experiment 2}

The model of motor control presented in Figure 8 brings some clarity to why alterations to the environment might only influence a portion of pointing movements in the current paradigm. Indeed, it is reasonable to suggest that a robust representation of the environment must be in place in order to plan interaction behaviours. Because motor control is composed of both a planning and an online monitoring (control) phase, it is possible for alterations to the 
representation of either the environment or the body to have differential effects on the way interaction behaviours, such as pointing, unfold. Environmental manipulations seem to alter the planning phase of pointing movements in the current experiment, but it is not clear whether manipulations to the virtual effector should have a similar influence on pointing movements. It is entirely possible that that effector manipulations have an effect on the planning phase of pointing movements, on the monitoring phase, on both phases, or on neither.

The role of effector representation on embodied presence was not directly manipulated Experiment 2. Experiment 3 therefore introduced a direct manipulation of the effector factor. Further, the design of Experiment 3 was such that environment and effector factors were simultaneously manipulated in order to examine the unique role of these two factors' in supporting embodied presence.

\section{Summary}

Experiment 2 examined the influence of environment factors on embodied presence by presenting virtual fire as participants made pointing movements. Liftoff times were longer in the presence of virtual fire, whereas travel times were unaffected. Based on models of motor control the findings suggest that environmental factors have their effects at the early motor planning phase and not the later motor control phase. Findings from Experiment 2 suggest that manipulations to the environment influence embodied presence. 


\section{EXPERIMENT 3: THE EFFECTS OF ENVIRONMENT VERSUS EFFECTOR ON EMBODIED PRESENCE}

The purpose of Experiment 3 was to examine the unique roles of environmental and effector factors on embodied presence. In the EPM, environment and effector are related in the threedirectional relationship between mind, body, and environment. It is possible that the environmental and effector factors contribute independently to embodied presence. In order to address this possibility, manipulations to both environment and effector were included in Experiment 3. As in Experiment 2, virtual fire versus. no-fire was used as an environment manipulation. For the effector manipulation, virtual cube versus virtual hand effectors were compared.

As shown in Experiment 2, fire in the virtual environment affected only liftoff times. This result suggests that environment factors have their effect on the earlier motor planning stage. It remains to be seen whether manipulations to the virtual effector act at this early planning stage as well, or a later execution stage, or potentially both stages. Given that the early stage of motor planning translates task demands into motor goals, it is expected that the effector factor should not affect motor planning, but rather, should influence the later motor control stage representing online monitoring and outcome accuracy. Effector influences at the later stage of motor control should cause an observable difference between the cube and hand effectors on travel time and landing accuracy. It is worth noting here that embodied presence in the EPM is assumed to support task performance, therefore better task performance in one effector condition over the other can mean stronger embodied presence. It is expected that virtual fire will affect the early motor planning stage and cause delays to liftoff times for both the cube and hand effectors in Experiment 3 as was seen in Experiment 2. If, however, effector influences the early motor 
planning stage, then there should be an interaction between fire and hand representation on liftoff times.

\section{METHODS}

\section{Participants}

Participants were fifty-five undergraduate students at Carleton recruited from the SONA research pool who participated in exchange for $1 \%$ bonus credit toward an eligible psychology course. As in Experiments 1 and 2, participants were asked about their experience with the VR technology but were not excluded for having prior exposure. Participants were right-handed, with normal or corrected-to-normal vision and with a neurological history free from any motor control difficulties or conditions predisposing them to nausea (see Appendix A: Experiment documents). Two participants were removed from the analysis due to experiment difficulties, resulting in the analysis of fifty-three participants.

\section{Apparatus}

The technical apparatus was identical to that of Experiment 2.

\section{Display and Stimuli}

Experiment 3 utilized the same virtual environment and reaching task as Experiments 1 and 2. Both a hand condition and a cube condition were included. For the hand condition participants saw the same high-fidelity hand model used in Experiment 2. For the cube conditions, participants saw a virtual cube as the representation of their fingertip. As compared to Experiment 1, the size of the virtual cube for Experiment 3 was reduced to $1.5 \mathrm{~cm}$ as a small representation of the fingertip of the reaching hand (see Figure 15). This size alteration relative to Experiment 1 was made to reduce occlusion of the target caused by the original $3 \mathrm{~cm}$ cube, 
and to take advantage of the Leap Motion controller's greater tracking capabilities which made it possible for participants to make a true pointing gesture.

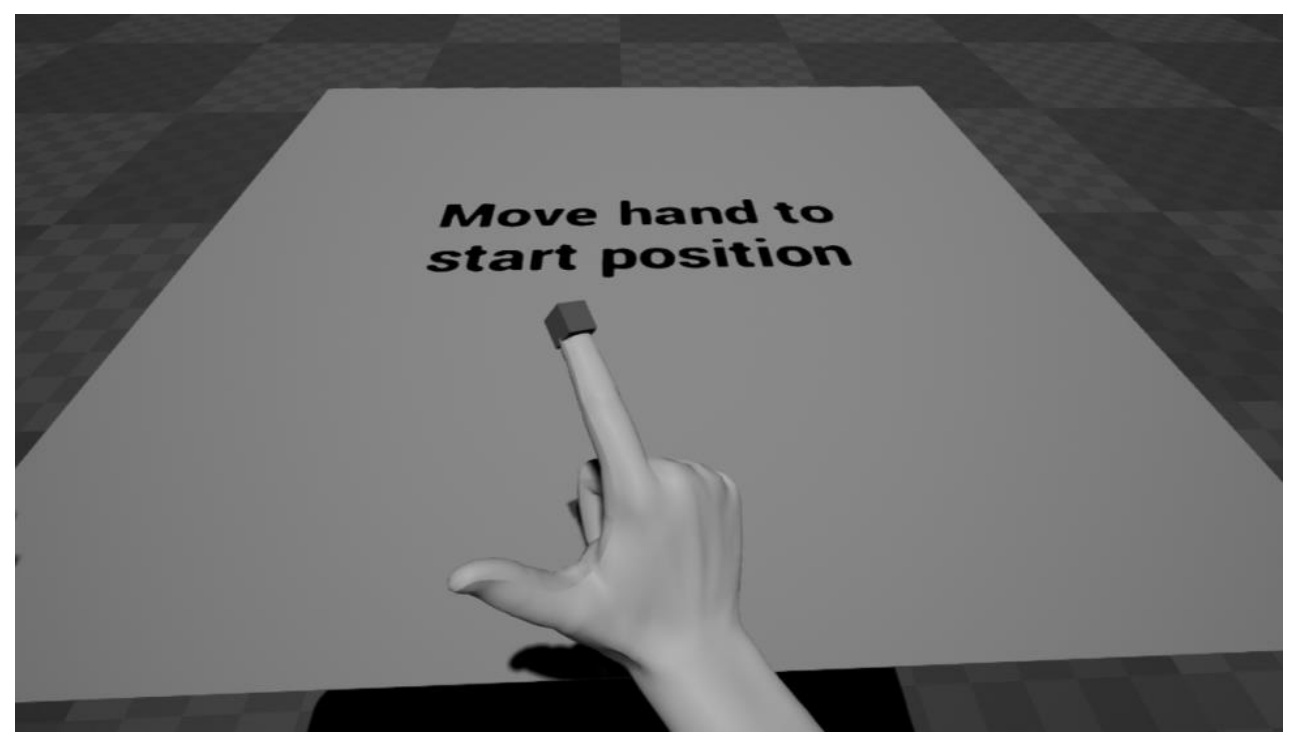

Figure 15. Cube representation of fingertip, note that the hand is visible in Figure for demonstration purposes but was not visible to participants during experiment).

\section{Design}

Experiment 3 utilized a 2 (Hand Representation: cube vs. hand) x 2 (Fire: none vs. near) x 5 (Target) repeated-measures design. Hand representation was divided into blocked trials that were counterbalanced such that half of the participants started the experiment with the cube block and half started with the hand block. Each block began with 20 practice trials. The presentation of fire was restricted to the subsequent 200 experimental trials in each block randomized at the trial level for a total of $30 \%$ of all trials for a total of 60 fire trials per each 200-trial block).

\section{Procedure}

The briefing, consent, and setup procedures and trial sequence were the same as those described for Experiments 1 and 2. Experiment 3 was broken into 2 halves for participants to 
have a rest and proceeded for approximately 30 minutes followed by the same questionnaire adapted from Experiment 1 and a post experiment interview.

\section{RESULTS}

\section{Pointing Data}

Latencies. Pointing movement data were trimmed according to the same procedure used in Experiments 1 and 2 resulting in a total 13.6\% data loss. The remaining liftoff time and travel times data were submitted to separate 2 (Hand Representation: cube vs. hand) x 2 (Fire: none vs. near) x 5 (Target) repeated measures ANOVAs.

The omnibus test results for liftoff time data are presented in Table 6. As predicted, and consistent with results from Experiment 2, there was main effect of Fire such that liftoff times were delayed when there was fire in the virtual environment $(471 \mathrm{~ms})$ as compared to when there was no fire (439ms). There was a significant main effect of Target. As shown in Figure 16, liftoff times were fastest for Targets 2 (454ms) and Target 4 (446ms) as compared to Target 1 (462ms), Target 4 (465ms) and Target 5 (446ms). There was also an interaction between Target Table 6. Omnibus effects on liftoff times for the 2 (Hand Representation: cube vs. hand) x 2 (Fire: none vs. near) x 5 (Target) repeated measures ANOVA.

\begin{tabular}{llrrrr}
\hline Effect & df (adjusted) & \multicolumn{1}{c}{ MS } & \multicolumn{1}{c}{$F$} & $\eta_{\mathrm{p} 2}$ \\
\hline Target & $4(3.5)$ & $18167.42 \mathrm{a}$ & 20.049 & $<.001$ & .282 \\
$\quad$ Error & $204(176.2)$ & $906.13 \mathrm{a}$ & & & \\
HandRep & 1 & 11736.10 & 1.57 & .216 & .030 \\
$\quad$ Error & 51 & 7475.16 & & & \\
Fire & 1 & 259327.71 & 56.052 & $<.001$ & .524 \\
$\quad$ Error & 51 & 4626.58 & & & \\
Target x HandRep & $4(3.4)$ & $285.713 \mathrm{a}$ & 0.601 & .662 & .012 \\
$\quad$ Error & $204(172.9)$ & $475.088 \mathrm{a}$ & & & \\
Target x Fire & 4 & 2527.68 & 4.611 & .001 & .083 \\
$\quad$ Error & 204 & 548.23 & & & \\
HandRep x Fire & 1 & 874.413 & 0.828 & .367 & .016 \\
$\quad$ Error & 51 & 1055.694 & & & \\
Target x HandRep x Fire & 4 & 806.962 & 1.614 & .172 & .031 \\
$\quad$ Error & 204 & 500.05 & & & \\
\hline
\end{tabular}

aGreenhouse-Geisser adjusted 


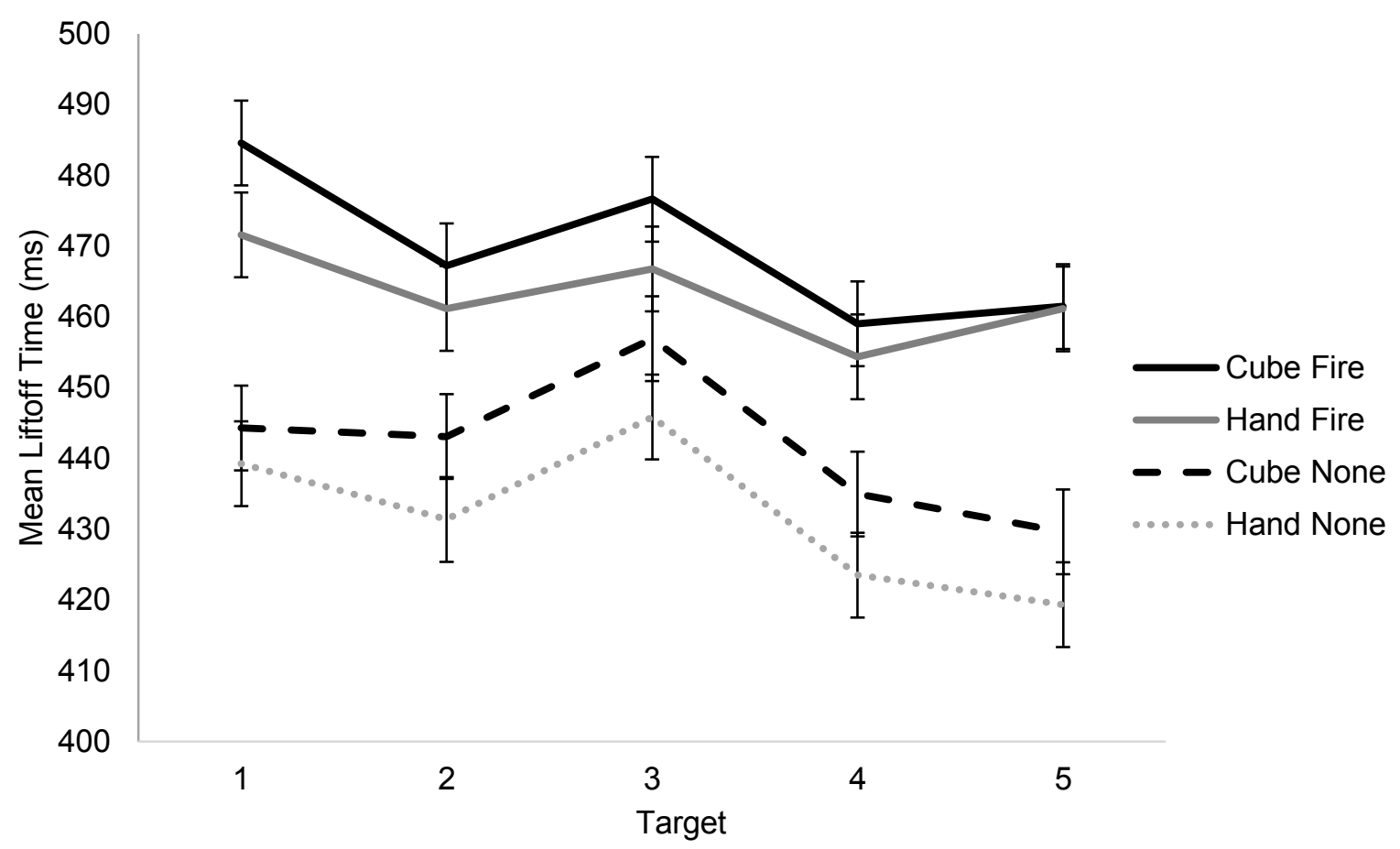

Figure 16. Mean liftoff times to all targets by Fire and Hand Representation. Error bars represent $95 \%$ CIs for the comparison at Hand Representation as per Hollands and Jarmasz, 2010.

and Fire such that the presence of fire resulted in more dramatic a delay in liftoff times for peripheral targets as compared to the centre target location (Target 3, see Figure 16).

Omnibus results on travel times can be found in Table 7. As predicted, there was a significant main effect of Hand Representation where travel times were shorter for the hand (464ms) than the cube representation (493ms). There was also a significant main effect of Target such that travel times to leftward targets were longer (Target 1: 502ms; Target 2: 493ms) than the central target (Target 3: 482ms) and the rightward targets (Target 4: 467ms; Target 5: 448ms; see Figure 17). This pattern of faster travel to rightward targets is consistent with Experiments 1 and 2. There were no other significant effects on travel times. 
Table 7. Omnibus effects on travel times for the 2 (Hand Representation: cube vs. hand) $\mathrm{x} 2$ (Fire: none vs. near) x 5 (Target) repeated measures ANOVA.

\begin{tabular}{llrrrr}
\hline Effect & df (adjusted) & \multicolumn{1}{c}{ MS } & \multicolumn{1}{c}{$p$} & $\eta_{\mathrm{p} 2}$ \\
\hline Target & $4(2.7)$ & $136480.61_{\mathrm{a}}$ & 166.824 & $<.001$ & .766 \\
$\quad$ Error & $204(139.8)$ & $818.109 \mathrm{a}$ & & & \\
HandRep & 1 & 219686.78 & 24.508 & $<.001$ & .325 \\
$\quad$ Error & 51 & 8964.00 & & & \\
Fire & 1 & 1355.30 & 1.164 & .286 & .022 \\
$\quad$ Error & 51 & 1164.72 & & & \\
Target x HandRep & $4(3.1)$ & $310.90 \mathrm{a}$ & 0.684 & .570 & .013 \\
$\quad$ Error & $204(160.3)$ & $454.62 \mathrm{a}$ & & & \\
Target x Fire & 4 & 373.49 & 1.02 & .398 & .020 \\
$\quad$ Error & 204 & 366.18 & & & \\
HandRep x Fire & 1 & 42.14 & 0.115 & .736 & .002 \\
$\quad$ Error & 51 & 367.41 & & & \\
Target x HandRep x Fire & $4(3.2)$ & $166.16 \mathrm{a}$ & 0.476 & .710 & .009 \\
$\quad$ Error & $204(348.8)$ & $348.75 \mathrm{a}$ & & & \\
\hline
\end{tabular}

aGreenhouse-Geisser adjusted

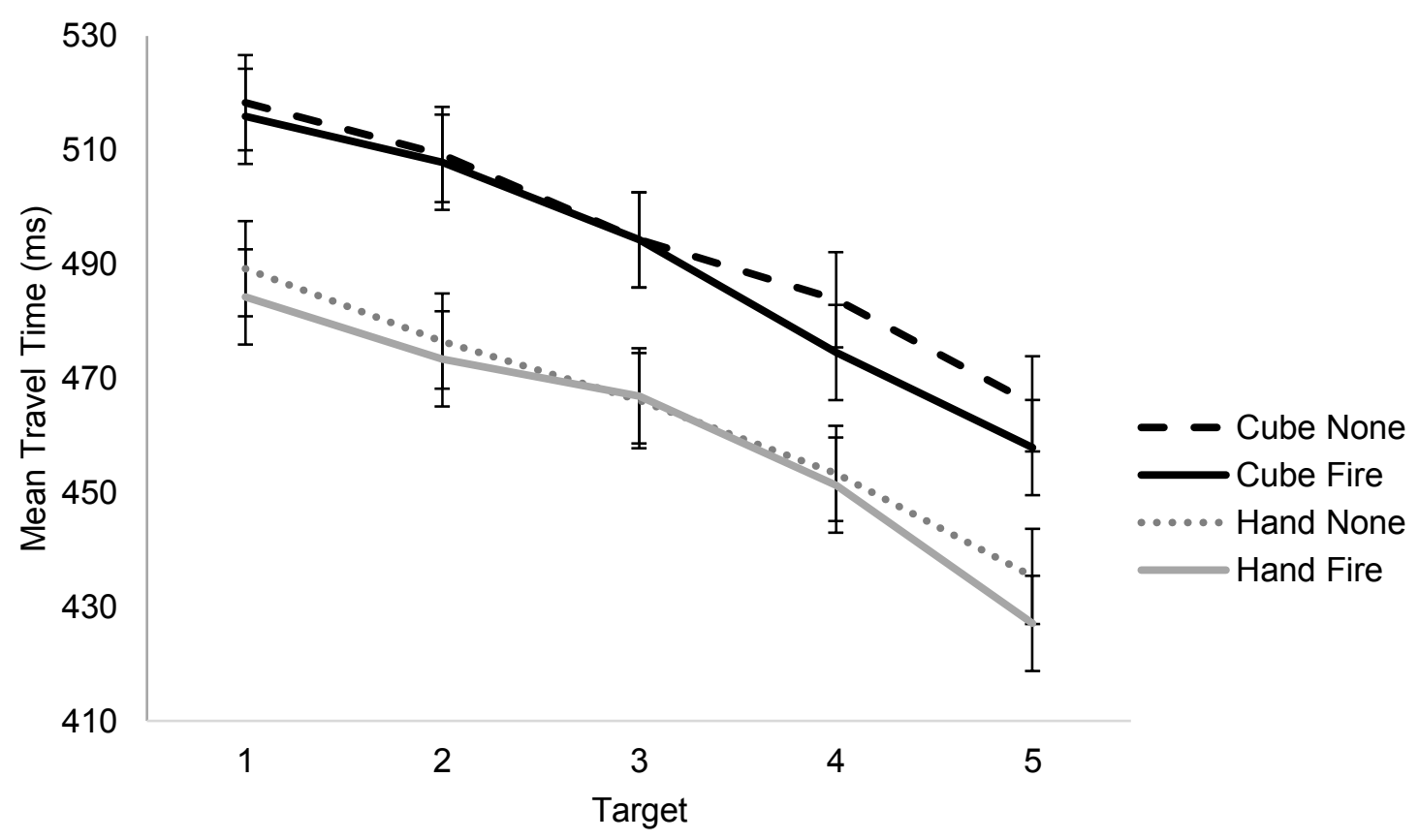

Figure 17. Mean travel times to all Targets to all targets by Fire and Hand Representation. Error bars represent 95\% CIs for the comparison at Fire as per Hollands \& Jarmasz, 2010. 
Accuracy. Pointing accuracy was examined using the same percent hit rate measure described in Experiment 1. Mean percent hit rate scores were submitted to a 2 (Hand Representation: cube vs. hand) x 2 (Fire: none vs. near) x 5 (Target) repeated measures ANOVA, results can be found in Table 8 . As predicted, there was a significant main effect of Hand Representation where percent hit rate was higher for the hand $(81 \%)$ than the cube representation (73\%). There was also a significant interaction between Target and Hand Representation. Figure 18 shows that the difference between hand and cube hit rates was attenuated for rightward targets as compared to leftward. No other statistically significant effects were present in the percent hit rate analysis.

Table 8 Omnibus effects on Percent hit rate for the 2 (Hand Representation: hand vs. cube) x 2 (Fire: none vs. near) x 5 (Target) repeated measures ANOVA.

\begin{tabular}{lcrccc}
\hline Effect & df (adjusted) & MS & $F$ & $p$ & $\mathrm{y}_{\mathrm{p} 2}$ \\
\hline Target & $4(2.8)$ & $382.831 \mathrm{a}$ & 1.448 & .233 & .028 \\
$\quad$ Error & $204(143.6)$ & $264.313 \mathrm{a}$ & & & \\
HandRep & 1 & 18954.20 & 8.409 & .005 & .142 \\
$\quad$ Error & 51 & 2254.12 & & & \\
Fire & 1 & 79.29 & 0.513 & .477 & .010 \\
$\quad$ Error & 51 & 154.56 & & & \\
Target X HandRep & $4(3.5)$ & $671.498 \mathrm{a}$ & 4.139 & .005 & .075 \\
$\quad$ Error & $204(176.4)$ & $162.224 \mathrm{a}$ & & & \\
Target x Fire & 4 & 83.62 & 0.773 & .544 & .015 \\
$\quad$ Error & 204 & 108.12 & & & \\
HandRep x Fire & 1 & 0.51 & 0.004 & .949 & .000 \\
$\quad$ Error & 51 & 125.92 & & & \\
Target x HandRep x Fire & 4 & 61.84 & 0.658 & .622 & .013 \\
$\quad$ Error & 204 & 94.04 & & & \\
\hline
\end{tabular}

aGreenhouse-Geisser adjusted 


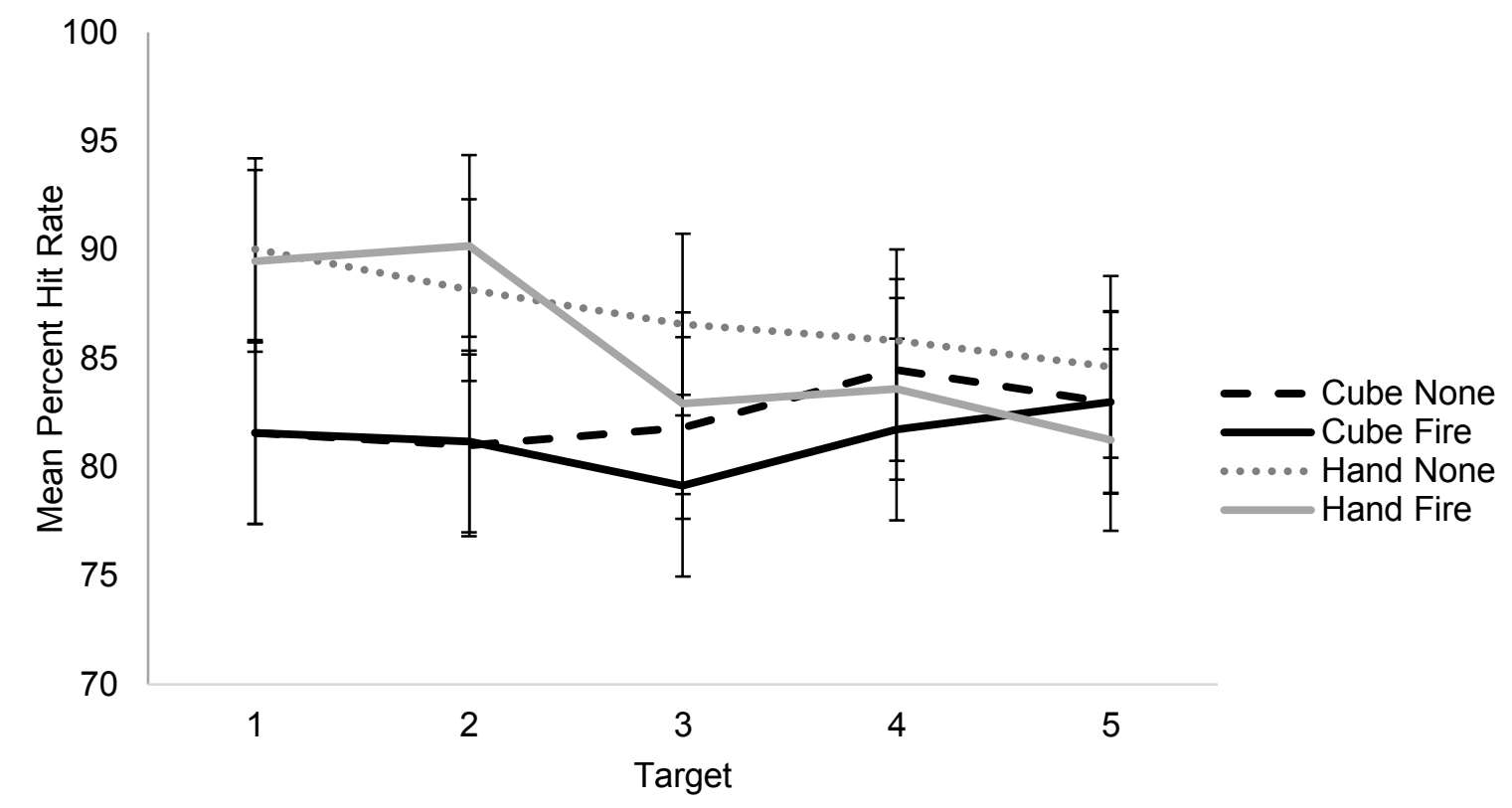

Figure 18. Mean Percent Hit Rate by Target, Fire, and Hand Representation. Error bars represent 95\% CIs for the comparison at the Hand Representation as per Hollands and Jarmasz, 2010.

\section{Questionnaire Data}

After each block participants completed the same questionnaire as in Experiments 1 and 2. The questionnaire was adapted to match the cube versus hand effector condition that had just been completed. Reponses were compared between the cube and hand effector conditions and can be found in Figure 19. Paired sample t-tests revealed no differences between the effector conditions on participants' responses on any of the questionnaire items (ps $>.05$ after Bonferroni adjustment for multiple comparisons. Responses follow the pattern observed Experiments 1 and 2 and suggest that participants were experiencing embodied presence. 


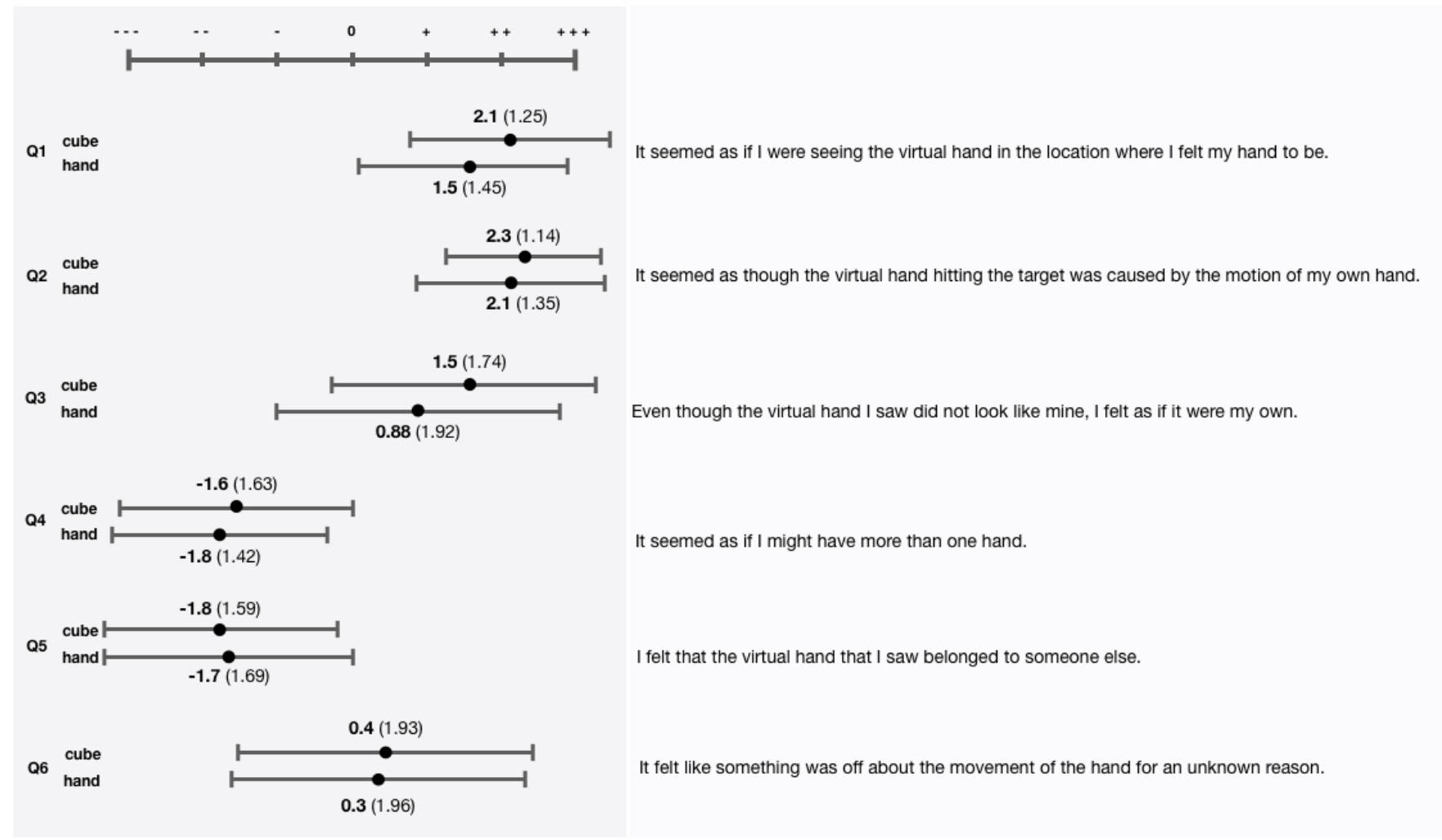

Figure 19. Mean responses to post experiment questionnaire where "- _ -" signified strong disagreement and " +++ " signified strong agreement and error bars represent standard deviation of the item mean.

\section{DISCUSSION}

Experiment 3 examined how embodied presence is affected by changes in a virtual environment and by the use of different effectors for interacting in the virtual environment. The environment was manipulated by adding virtual fire, which resulted in changes to liftoff times.

Participants took longer to initiate pointing movements when there was fire in the virtual environment compared to when there was none. This finding is consistent with Experiment 2 where a difference between near and far fire conditions demonstrated that the effect of fire is not a mere novelty (startle) effect. The effect of fire delaying liftoff times in both Experiments 2 and 3 is taken as evidence for embodied presence insofar as participants hesitation to initiate pointing suggests they may have experienced concern for their virtual effectors. 
Effector was manipulated in Experiment 3 by providing a virtual cube versus virtual hand for the pointing task. Differences in virtual effector changed travel times toward targets: pointing movements to targets were slower when using the cube effector than the hand effector. This finding is consistent with other studies of pointing behaviour in which similar disturbance patterns have been found when participants are asked to point with a representative point at the tip of their index finger as compared to full sight of their hand (see Henriques \& Cressman, 2012 for a review). There was also a degradation in pointing accuracy for the cube as compared to the hand effector in Experiment 3. The influence of virtual effector on travel times and landing accuracy suggest that the representation of the body is important for virtual task performance. When examined in conjunction with the results of the questionnaire, the changes in performance demonstrate that participants experienced embodied presence in the virtual pointing task.

The finding of effector influencing pointing performance in Experiment 3 extends those of Experiment 1. In Experiment 1 the virtual representation of the effector remained as a cube while the physical hands were manipulated into right- and left-hand conditions. Conversely, in Experiment 3 the virtual representation of effector was manipulated into cube versus hand conditions while the task was performed with participants' right hands only. Although it would have been reasonable to suggest that only manipulations to the physical effectors (left vs. right hands) would result in changes to performance, manipulations to the virtual representation of effectors in Experiment 3 also influenced pointing performance. Taken together, the findings from Experiments 1 and 3 suggest that the mental representation of the body remains intact in virtual environments.

An important finding from Experiment 3 is that the environment and effector factors did not interact. Environment manipulations affected motor planning in the form of longer liftoff 
times when fire was present. Effector manipulations influenced motor control in the form of longer travel times for the cube than the hand effector. This pattern of separate influences on difference stages of motor control supports the notion that environment and effector have independent influences on embodied presence (see Figure 20). In summary, Experiment 3 demonstrated a replication of previous findings that effector manipulations alter pointing movements, and extended these findings to suggest that effector uniquely influences later execution stage of motor control. The findings of Experiment 2 were also replicated and extended to suggest that manipulations to the virtual environment uniquely affect the early planning stage of motor control.

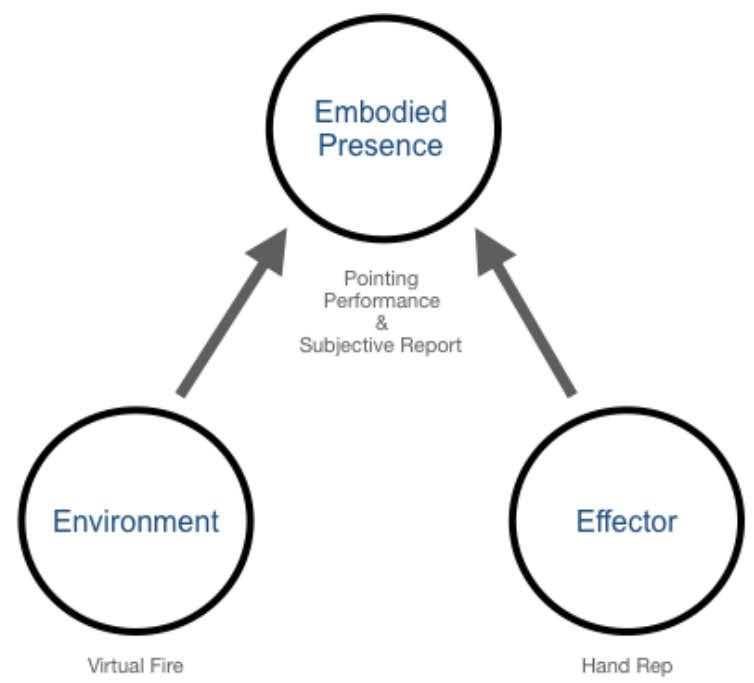

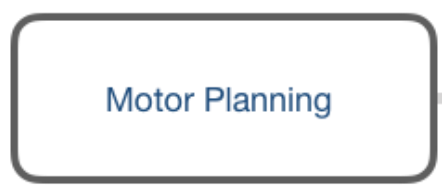

Gathering task requirements, formation of motor goals, plan for movement execution

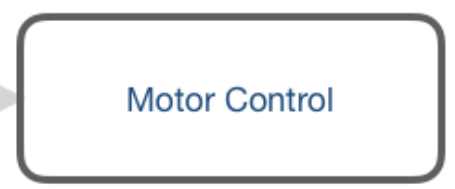

Forward predictions of movement outcome, online feedback for adjustments

Figure 20. Update to the EPM with findings from Experiment 3 mapped against the simplified model of motor control presented in Experiment 2. A lack of interaction between environment and effector on pointing movement kinematics suggests environment and effector are independent influences on embodied presence. 


\section{GENERAL DISCUSSION}

The present research examined the role of environment and effector factors on presence in virtual reality. An Embodied Presence Model (EPM) was proposed in which the relationship between mind, body, and environment is fundamental to the experience of presence in virtual reality. A fundamental assumption in the EPM is that presence is a direct result of interactions between one's surroundings and one's self. This assumption is based in the current understanding of how the brain constructs separate representations for the environment and for the self. Figure 20 shows how the EPM represents interactions between surroundings and self as environment and effector, respectively.

In a series of three experiments, environment and effector manipulations were found to influence embodied presence as reflected in both task performance and subjective reports. In each experiment participants were required to point toward virtual targets using a virtual representation of their hand. This virtual effector was either a cube or a high-fidelity hand model depending on the experimental conditions. Two main behavioural outcome measures were derived from pointing movements: liftoff time was calculated as the amount of time it took participants to initiate pointing after the target appeared, and travel time was calculated as the amount of time it took participants to reach the target after liftoff. Landing position was also examined to verify accurate pointing performance and to complement analyses of the latency measures. Post-experiment questionnaires provided a self-report measure of participants' subjective experience of embodied presence. Across all three experiments participants reported experiencing embodied presence in that they agreed with questionnaire items that described a strong relation between their movements and those of the virtual effectors. Taken together the 
behavioural and self-report data demonstrate the connection between virtual environment and effector and the subjective experience of being present and able to interact with a virtual space.

Experiment 1 examined participants' pointing movements to test whether the representation of the body and its effectors remains intact in virtual settings. As has been reported with reaching movements in natural environments, liftoff times were faster for left than right hands. This finding supports the notion that the virtual left- and right-hand cube effectors were represented in the same manner as hands are in the physical world. Additional support for this claim was found in the travel time measure, where the right-hand traveled to targets more quickly than the left-hand. Travel times were also quicker to targets in a hand's own hemispace, which aligns with findings found with reaching in natural space (Fisk \& Goodale, 1985; see also Boulinguez et al., 2001a; 2001b). In sum, the differences between left- versus right-hand pointing movements in Experiment 1 provides evidence that a similar mental representation of the body and its effectors exists when participants are reaching to targets in virtual environments.

Experiment 2 examined the role of environment on embodied presence by introducing virtual fire into the environment at the beginning of a subset of trials. It was found that fire slowed liftoff times, and that fire located near participants delayed liftoff times more than fire far away from the participants. In contrast, travel times were not affected by virtual fire. This finding supported the notion that the representation of the environment affects motor planning in the earlier phase of executing movements, as models of motor control would predict (see Wong et al., 2015). Delayed liftoff times were also taken as evidence of embodied presence; participants hesitated to initiate pointing because they were concerned for the well-being of their virtual effectors. In sum, longer liftoff times in the presence of virtual fire provide evidence that a 
representation of the environment affects embodied presence via the early motor planning phase of movement execution.

Experiment 3 examined the effect of environment in the virtual pointing task by presenting virtual fire and introducing cube versus hand effector conditions. Liftoff times were delayed in the presence of virtual fire, while travel times were not. This finding replicates Experiment 2 and provides further support for the notion that the representation of environment influences the early motor planning phase of executing movements. In Experiment 3, the role of effector on pointing behaviour was examined by comparing performance between virtual hand and a virtual cube effector. Travel times were slower and less accurate with the virtual cube effector than the virtual hand. Liftoff times were unaffected by type of effector. In sum, introducing virtual fire into the environment only affected liftoff times. In contrast, the impact of changing the effector from a virtual cube to a virtual hand was only reflected in travel times. This pattern of separate influences supports the notion that environment and effector influences different stages of motor control and highlights the possibility that these factors support embodied presences independently.

\section{A serial relationship between environment and effector}

Separate manipulations to environment and effector factors resulted in separate effects on the two stages of executing pointing movements. Experiment 3 in particular highlights this pattern. Manipulations of environment influenced the way participants initiated pointing movements (motor planning) but left subsequent movement characteristics unaffected. Conversely, manipulations of effector influenced the way pointing movements unfolded (motor control) while not affecting the initiation of movements. Due to the serial nature of motor planning and motor control, the pattern of results raises the possibility that there may be a serial 


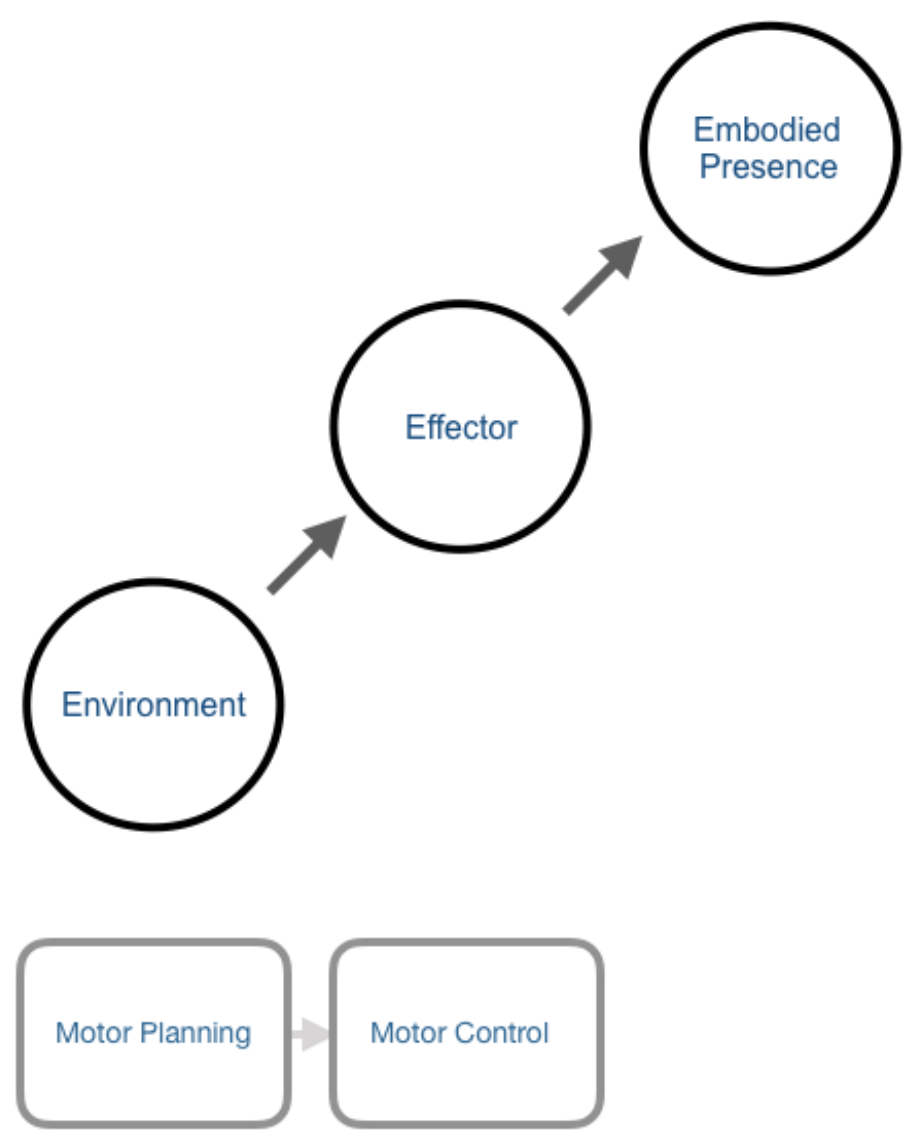

Figure 21. A serial version of EPM with a depiction of the motor control model as supporting evidence presented at bottom in square boxes.

relationship between the factors themselves. That is, first a representation of the virtual environment may be constructed, followed by a representation of the virtual body and its effectors. This logic suggests that the EPM should be organized as shown in Figure 21.

An alternative account might be that the apparent serial relationship between the influence of environment and effector is an artifact of the task used in the present methodology. Indeed, the motor-based task used in the present work is certainly supported by the well-studied serial-stage model of motor control. Other researchers, however, have postulated that the emergence of presence is a 2-stage process (e.g., Hofer et al., 2012; Wirth et al., 2007). Hofer et al. suggested that presence is supported first by the construction of a spatial situational model and second by the emergence of spatial presence. By their own admission, the self-report 
methods used by these researchers prevented them from directly testing the serial nature of these factors supporting presence. The behavioural evidence from the present research, and in particular Experiment 3, is consistent with the serial stages of presence that these researchers hypothesized. Notable difference in the conceptualization of the factors supporting presence are discussed in the next section.

The neuropsychological literature that EPM was informed by provides less clear evidence either to support or refute a serial organization of the EPM. Given that neurological representations of the environment and the body are dynamically re-coded with incoming sensory information at a near constant rate, it is difficult to infer the order in which these updates are prioritized. The representation of the body, the body schema, is often described as an online model of current bodily state (Caruthers, 2008). The mental representation of the environment is also updated constantly, as evidenced by the immediate recoding of reachable space by changes to proprioceptive inputs provided by using reaching tools (e.g., Berti \& Fracinetti, 2000; Iriki et al., 1996). It should be noted, however, that both of these neurological re-code processes emerge via the connection between representations of both environment and body. For example, the recoding of reachable space demonstrated by Berti and Fracinetti (2000) resulted from proprioceptive (bodily) inputs from a reaching tool as compared to a laser pointer, which suggests that, serial or not, the representations of environment and body are interconnected. To definitively examine the potential serial nature of the EPM would require a different experimental task and outcome measures.

\section{Environment and effector in other models of presence}

Environment and effector are represented in other models of presence in the literature. To date the closest model to the EPM is one of the first, from Loomis (1992), where presence is 
described as the sensorimotor relationship between (1) the nervous system and its internal model of the world and the self with (2) the virtual or physical world via a body and its effectors. The relationship between internal world and external is modeled as a linkage made possible by effectors, and Loomis defines that "true presence" as having no awareness of the linkage. Other early models of presence were focused on the ability of the mediating technology to present convincing sensory information about the virtual environment to the observer (e.g., Heeter, 1992; Sheridan, 1992) while later models focused on the ability of a virtual environment to provide richer experiences (e.g., Schubert et al., 2001, 2001; Witmer \& Singer, 1998). Slater (2009) eventually split his conceptualization of presence into two constructs: (1) a place illusion wherein the observer experiences a sense of self-location, or "being there" in the VE, and (2) a plausibility illusion wherein the observers expectations about cause and effect, such as social exchanges, are upheld. Researchers have also started including higher order cognitive factors and individual differences (e.g., Hofer et al., 2012; Weibel \& Wissmath, 2011) as factors contributing to a sense of presence. The EPM is unique from most models of presence in that it represents a return to modeling the perceptual-level phenomena that earlier researchers focused on, particularly those occurring at the sensorimotor system via the virtual effectors. This point of view is echoed in a recent reminder from Slater (2018) that presence is an illusion of perception, and not cognition; the observer never truly believes they have left the real world.

The EPM is unique in that environment and effector are viewed as making separate contributions to embodied presence. Most models of presence have a representation of effector conflated with environment into a construct indexing the ability to interact with the virtual environment (see Table 9). Accordingly, researchers have typically focused on the extent of 
Table 9. Factors of environment, effector, and their interaction in other models of presence in the literature.

\begin{tabular}{llll}
\hline Model of Presence & Environment & Effector & $\begin{array}{l}\text { Environment } x \text { Effector } \\
\text { (Embodied Presence) }\end{array}$
\end{tabular}

\begin{tabular}{|llll} 
Heeter, 1992 & $\begin{array}{l}\text { Subjective Personal } \\
\text { Presence }\end{array}$ & $\begin{array}{l}\text { Environmental Presence } \\
\text { (manipulate) }\end{array}$ \\
\hline Loomis 1992 & $\begin{array}{l}\text { Representation of External } \\
\text { World }\end{array}$ & $\begin{array}{l}\text { Representation of } \\
\text { Phenomenal Self }\end{array}$ & $\begin{array}{l}\text { Linkage between worlds via } \\
\text { effectors }\end{array}$ \\
\hline
\end{tabular}
$\begin{array}{lll}\text { Sheridan } 1992 & \text { Control relation of sensors } & \text { Ability to modify environment }\end{array}$ \& environment

Kim \& Biocca $1997 \quad$ Arrival \& Departure

Zahoric \& Jenison 1998 Suppression, Attentional Readiness-to-hand focus

$\begin{array}{lll}\text { Witmer \& Singer } 1998 & \text { Immersion } & \text { Involvement }\end{array}$

$\begin{array}{lll}\text { Bystrom et al. } 1999 \quad \text { Immersion } & \text { Attention to task and }\end{array}$

Sensory Fidelity

Performance

Shubert et al. 1999 \& Spatial Presence Involvement

2001

Realism

Wirth et al. $2007 \quad$ Self-location

Perceived possibility for action

Slater, 2009

Place Illusion

Plausibility Illusion

Hofer et al. $2012 \quad$ Self-location

Possible Actions

Skarbez et al., $2018 \quad$ Immersive qualities

Coherence of actions and consequence 
sensory input, motor output, and the consequences of intended actions such as the ability to modify the virtual environment rather than the congruity with which those inputs and outputs are experienced (e.g., Sheridan, 1992). This focus on the ability to interact with a virtual environment rather than the effectors supporting interactions conflates the representations of both environment and effector - although Gilbert (2017) has offered the concept of "authenticity", a congruent relationship between affordances in the VE and the outcome of intentions and actions, as an important consideration for presence. As previously noted, a fundamental assumption in the EPM is that embodied presence is a result of the interactions of one's self and one's surroundings, which necessitates a representation of both self and surrounding. One of the contributions the EPM offers to the presence literature is the point of view that the sensory system and its ability to inform mental representations of the body must be accounted for when examining presence.

Perhaps due to technology constraints, early models of presence were more likely to consider the relationship between the virtual environment and the teleoperative effectors that people could use to interact with virtual world. Zeltzer's (1990) presentation cited by Sheridan 1992) noted that others have combined these two factors together as 'user interaction'. Similarly, Glenberg's (1997) concept of meshed patterns is relied on as an explanation of how perception for action might support our sense of presence in a virtual environment (e.g., Schubert et al., 2001, Zahorik \& Jenison, 1998). This trend can be seen in later models of spatial presence (e.g., Wirth et al. 2007) where the representations of the environment and the body are combined across the two concepts of self-location and perception of possibility for action. It makes a certain amount of sense to combine these representations into a single construct under an embodied cognition framework, where thought itself is considered to be distributed across mind, 
body, and environment (Gibbs, 2006). By combining the representations of environment and body into a single construct for interaction, researchers lose the ability to separately examine breaks in presence due to either environmental or bodily factors. This becomes particularly important when VR is applied in clinical or training settings.

\section{The EPM Applied}

The EPM has direct implications for real-world applications of VR technologies. VR has been the focus of clinical research including phobia intervention (e.g., Carlin, Hoffman, \& Weghort, 1997; see also Botella, Fernández-Álvarez, Guillén, García-Palacios, \& Baños, 2017 for a recent review). VR provides a useful mechanism for exposing human participants to novel situations in a safe setting. As such, VR also presents an alternative for training personnel in safety-critical domains such as flying or high-risk domains such as surgery (e.g. Triantafyllou, Lazaridis, \& Dimitriadis, 2014). When creating training experience, virtual or otherwise, it is critically important to consider transfer of training, as research has demonstrated that the lowlevel cognitive skills learned in simulations such as video games transfer directly to real-world tasks such as driving (Green \& Bavalier, 2004). One of the advantages of the EPM is that it encourages researchers to consider the trainees' interactions with VR simulations as a result of mentally representing both the virtual environment and the virtual effectors. By making explicit the separate representations of environment and effector, it becomes possible to diagnose breaks in presence by examining task performance. Examining whether performance degrades in planning versus execution phases can help to determine whether improvements to the fidelity of the virtual environment are needed, or whether the representation of one's effectors is suboptimal.

\section{Future work}


The EPM could benefit from future studies that examine the trajectories of participants' pointing movements. Due to hardware and programming constraints in the present study the motion data were not collected in full 3-Dimensional fidelity. Specifically, information about the position of the tracked hand along the vertical axis was transformed so that the virtual representations of hands would never fall below the virtual table or move egregiously far outside the virtual workspace upon the table. When switching to the Leap Motion setup in Experiments 2 and 3 there were new issues that arose due to the nature of optical tracking. Because the camera was mounted to the virtual headset, the relative frame of reference moved when participant made pointing movements. This moving frame of reference was not cause for concern regarding liftoff and travel times, but it did require software intervention to ensure that a stable experience of viewed hand location during pointing movements. For this reason, there would have been variable expansion or shrinkage to the measurement of physical pointing trajectories. Introducing a separate hand tracking system would allow for the collection of truer pointing trajectories for analysis. Research has demonstrated that mid-flight changes to planned movement trajectories can be observed as delayed arrival of certain parts of the hand, such as the thumb, to the target posture for the new target trajectory (Till, Masson, Bub, \& Driessen, 2014). With enough tracking points it would possible to examine the finer changes to pointing movement associated with the different virtual effectors and the presentation of virtual fire.

The effects of fire on pointing behavior is currently interpreted as representing some degree of embodiment; participants hesitate to make pointing movements in the presence of fire because they are concerned for the well-being of their virtual effectors in the presence of a noxious stimulus. Indeed, the effect of fire was stronger when participants had to point through fire (near condition) compared to when they had to point in the presence of fire (far condition), 
thus ruling out a novelty effect. An alternative account for this finding might be that the effect of fire is in fact a capture of visual attention. On this view, delayed liftoff times in the presence of fire may indicate an inhibition of return (Posner \& Cohen, 1984) whereby attentional redeployment toward targets is downregulated after attentional capture by the fire stimulus. The present data do not lend themselves to a test of this alternative account, therefore a follow-up experiment could explore the nature of the fire effect. The same virtual pointing paradigm could be altered to include a control stimulus such as a bar of flashing lights at the same locations of the virtual fire in front of and behind the target locations. If the same delay of liftoff times is observed it may be the case that the effect of fire is attentional in nature. If, however, there is an observable difference between the fire and the light stimuli it could indicate an effect of embodiment whereby participants hesitate to point near fire because it is noxious whereas lights are not. Further, it might prove valuable to include neutral stimuli such as fog that do not carry a noxious status like fire but maintain similar movement patterns and occupy a similar spatial plane as the virtual fire.

In addition to these experimental changes it may prove useful to include some of the standard presence questionnaires (e.g. SUS, Slater, Usoh, \& Steed, 1994; IPQ, Schubert et al., 2001; Witmer \& Singer, 1998)) in order to capture a more qualitative perspective of participants' experience of presence. This would allow for the comparison of the subjective experience of being there and the experience of embodying a virtual effector. Schwind, Knierim, Haas, and Henze (2019) have demonstrated that it may be valuable to collect these subjective data in the virtual environment to reduce variability.

Future studies of the EPM could also introduce an intermixed method of presenting versions of the virtual effector and the virtual environment such that they were unknown until the 
start of the trial. This would mean that participants had to form a representation of the virtual environment and their virtual effectors on every trial. Manipulations at the trial level present the opportunity to examine the role of attention and other cognitive factors by using an alternatingruns procedure (Rogers \& Monsell, 1995). Switch costs for mentally representing one's effectors would open an inquiry into the role of executive function required to build or maintain a representation of the body. It would be interesting to observe a switch cost for building representations of the environment insofar as visual processes such as figure-ground assignment are thought to occur early and without attention (Julesz, 1984; Kimchi \& Peterson, 2008). Manipulating environment and effector at the task level instead of blocked conditions would provide an opportunity to present a wider variety of virtual environments and effectors.

\section{Conclusion}

The present research provides behavioural evidence that mental representations of the environment and of the body and its effectors influence embodied presence in virtual reality. The pattern of findings suggest that environment and effector factors are independent and possibly serial in nature. The Embodied Presence Model was proposed as a framework to articulate this relationship. The EPM not only captures the evidence provided in the present three experiments but also captures the constructs that many other presence researchers include in their models. The experimental paradigm developed in the present study provides a future opportunity to examine the role of cognitive factors in embodied presence and a means by which to examine the effects of mentally representing and interacting with what would otherwise be impossible bodies in impossible environments. By examining behavioural effects in virtual settings we can gain a deeper understanding of the psychological underpinnings of symptoms we wish to treat in patients or behaviours we wish to train in personnel. By exploring the boundaries of embodied 
presence, we not only move toward understanding the nature and consequence of mentally representing and simulating all that surrounds us, but we also move closer to understanding what it might take to transform "being there" into "being here". 


\section{REFERENCES}

Adams, J. A. (1971). A closed-loop theory of motor learning. Journal of Motor Behavior, 3(2), $111-150$.

Balslev, D., Cole, J., \& Miall, R. C. (2007). Proprioception contributes to the sense of agency during visual observation of hand movements: Evidence from temporal judgments of action. Journal of Cognitive Neuroscience, 19(9), 1535-1541.

https://doi.org/10.1162/jocn.2007.19.9.1535.Proprioception

Berti, A., \& Frassinetti, F. (2000). When far becomes near: Remapping of space by tool use. Journal of Cognitive Neuroscience, 12(3), 415-420.

https://doi.org/10.1162/089892900562237

Biocca, F. (1997). The cyborg's dilemma: Progressive embodiment in virtual environments. Journal of Computer-Mediated Communication, 3, 12-26. https://doi.org/10.1109/CT.1997.617676

Botella, C., Fernández-Álvarez, J., Guillén, V., García-Palacios, A., \& Baños, R. (2017). Recent Progress in Virtual Reality Exposure Therapy for Phobias: A Systematic Review. Current Psychiatry Reports, 19(7). https://doi.org/10.1007/s1 1920-017-0788-4

Botvinick, M., \& Cohen, J. (1998). Rubber hands "feel" touch that eyes see. Nature, 391(6669), 756. https://doi.org/10.1038/35784

Boulinguez, P., Velay, J. L., \& Nougier, V. (2001). Manual asymmetries in reaching movement control. II: Study of left-handers. Cortex, 37(1), 123-138. https://doi.org/10.1016/S00109452(08)70562-8

Boulinguez, P., Velay, J. L., \& Nougier, V. (2001). Manual asymmetries in reaching movement control. I: Study of right-handers. Cortex, 37(1), 101-122. https://doi.org/10.1016/S00109452(08)70562-8

Brown, L. E., Morrissey, B. E., \& Goodale, M. A. (2009). Vision in the palm of your hand. Neuropsychologia, 47, 1621-1626.

Bryden, M. P. (1982). Laterality functional asymmetry in the intact brain. New York, Academic Press.

Bystrom, K. E., Barfield, W., \& Hendrix, C. (1999). A conceptual model of the sense of presence in virtual environments. Presence: Teleoperators and Virtual Environments, 8(2), 241-244. https://doi.org/10.1162/105474699566107 
Carlin, A. S., Hoffman, H. G., \& Weghorst, S. (1997). Pergamon S0005-7967(96)00085-X SHORTER COMMUNICATIONS Virtual reality and tactile augmentation in the treatment of spider phobia: a case report. Behav. Res. Ther, 35(2), 153-158.

Carruthers, G. (2008). Types of body representation and the sense of embodiment. Consciousness and Cognition, 17(4), 1302-1316. https://doi.org/10.1016/j.concog.2008.02.001

Elliott, D., Roy, E., \& Goodman, D. (1993). Asymmetries in the preparation and control of manual aiming movements. Canadian Journal of ..., 22(3), 327-346. https://doi.org/10.1037/h0078856

Fisk, J. D., \& Goodale, M. A. (1985). The organization of eye and limb movements during unrestricted reaching to targets in contralateral and ipsilateral visual space. Experimental Brain Research, 60(1), 159-178. https://doi.org/10.1007/BF00237028

Gibbs, R. (2006). Embodiment and cognitive science. Cambridge University Press.

Gilbert, S. (2017). The Effects of Spatialized Sounds on the Sense of Presence in Auditory Virtual Environments: A Psychological and Physiological Study. Presence: Teleoperators and Virtual Environments, 25(4), 322-324. https://doi.org/10.1162/PRES

Glenberg, A. (1997). What is memory for. Behavioral and Brain Sciences, 20, 1-55. https://doi.org/10.1016/B978-0-08-097086-8.51059-9

Graziano, M. S. A., Yap, G. S., \& Gross, C. G. (1994). Coding of visual space by premotor neurons. Science, 266(5187), 1054-1057. https://doi.org/10.1126/science.7973661

Green, C. S., \& Bavelier, D. (2004). The cognitive neuroscience of video games.

Green, M. C., \& Brock, T. C. (2000). The role of transportation in the persuasiveness of public narratives. Journal of Personality and Social Psychology, 79(5), 701-721. https://doi.org/10.1037/0022-3514.79.5.701

Guterstam, A., Abdulkarim, Z., \& Ehrsson, H. H. (2015). Illusory ownership of an invisible body reduces autonomic and subjective social anxiety responses. Scientific Reports, 5(9831), 1-8. https://doi.org/10.1038/srep09831

Heeter, C. (1992). Being there: The subjective experience of presence. Presence: Teleoperators and Virtual Environments, 1, 262-271.

Henriques, D. Y. P., \& Cressman, E. K. (2012). Visuomotor adaptation and proprioceptive recalibration. Journal of Motor Behavior, 44(6), 435-444.

https://doi.org/10.1080/00222895.2012.659232 
Hofer, M., Wirth, W., Kuehne, R., Schramm, H., \& Sacau, A. (2012). Structural Equation Modeling of Spatial Presence: The Influence of Cognitive Processes and Traits. Media Psychology, 15(4), 373-395. https://doi.org/10.1080/15213269.2012.723118

IJsselsteijn, W. A., de Ridder, H., Freeman, J., \& Avons, S. E. (2003). Presence: Concept, determinants and measurement. In Human Vision and Electronic Imaging V (pp. 520-529). https://doi.org/10.1117/12.387188

Iriki, A., Tanaka, M., \& Iwamura, Y. (1996). Coding of modified body schema during tool use by macaque postcentral neurones. NeuroReport, 7(14), 2325-2330.

Jackson, P. L., Rainville, P., \& Decety, J. (2006). To what extent do we share the pain of others? Insight from the neural bases of pain empathy. Pain, 125(1-2), 5-9. https://doi.org/10.1016/j.pain.2006.09.013

Jeannerod, M. (2001). Neural simulation of action: A unifying mechanism for motor cognition. NeuroImage, 14(1), 103-109. https://doi.org/10.1006/nimg.2001.0832

Julesz, B. (1984). A brief outline of the texton theory of human vision. Trends in Neurosciences, $7(2), 41-45$.

Keele, S. W. (1968). Movement in skilled motor performance. Psychological Bulletin, 70(6), 387-403. Retrieved from http://grants.hhp.coe.uh.edu/clayne/HistoryofMC/Keele1968.pdf

Kim, T., \& Biocca, F. (1997). Telepresence via television: Two dimensions of telepresence may have different connections to memory and persuasion. Journal of Computer-Mediated Communication, 3(2).

Kimchi, R., \& Peterson, M. A. (2010). Figure-ground segmentation can occur without attention. Journal of Vision, 8(6), 825-825. https://doi.org/10.1167/8.6.825

Klasen, M., Weber, R., Kircher, T. T. J., Mathiak, K. A., \& Mathiak, K. (2012). Neural contributions to flow experience during video game playing. Social Cognitive and Affective Neuroscience, 7(4), 485-495. https://doi.org/10.1093/scan/nsr021

Lombard, M., \& Ditton, T. (2006). At the Heart of It All: The Concept of Presence. Journal of Computer-Mediated Communication, 3(2). https://doi.org/10.1111/j.10836101.1997.tb00072.x

Loomis, J. M. (1992). Distal Attribution and Presence. Presence: Teleoperators and Virtual Environments, 1(1), 113-119. https://doi.org/10.1162/pres.1992.1.1.113

Minksy, M. (1980). Telepresence. Omni, 45-51. 
Pan, X., \& Hamilton, A. F. d. C. (2018). Why and how to use virtual reality to study human social interaction: The challenges of exploring a new research landscape. British Journal of Psychology, 109(3), 395-417. https://doi.org/10.1111/bjop.12290

Petkova, V. I., \& Ehrsson, H. H. (2009). When right feels left: Referral of touch and ownership between the hands. PLoS ONE, 4(9). https://doi.org/10.1371/journal.pone.0006933

Rizzolatti, G., Fadiga, L., Gallese, V., \& Fogassi, L. (1996). Premotor cortex and the recognition of motor actions. Cognitive Brain Research, 3(2), 131-141. https://doi.org/10.1016/09266410(95)00038-0

Rizzolatti, G., Scandolara, C., Matelli, M., \& Gentilucci, M. (1981). Afferent properties of periarcuate neurons in macaque monkeys. I. Somatosensory responses. Behavioural Brain Research, 2(2), 125-146. https://doi.org/10.1016/0166-4328(81)90052-8

Rizzolatti, G., Scandolara, C., Matelli, M., \& Gentilucci, M. (1981). Afferent properties of periarcuate neurons in macaque monkeys II: Visual responses. Behavioural Brain Research, 2, 147-163. https://doi.org/10.1016/j.genhosppsych.2011.10.009

Rogers, R. D., \& Monsell, S. (1995). Costs of a Predictable Switch Between Simple Cognitive Tasks. Journal of Experimental Psychology: General, 124(2), 207-231. https://doi.org/10.1037/0096-3445.124.2.207

Schubert, T., Firedmann, F., \& Regenbrecht, H. (1999). Embodied Presence in Virtual Environments. In Visual representations and interpretations (pp. 269-278). Springer.

Schubert, T., Friedmann, F., \& Regenbrecht, H. (2001). The experience of presence: Factor analytic insights. Presence: Teleoperators and Virtual Environments, 10(3), 266-281. https://doi.org/10.1162/105474601300343603

Schuemie, M. J., Van Der Straaten, P., Krijn, M., \& Van Der Mast, C. A. P. G. (2001). Research on Presence in Virtual Reality: A Survey. CyberPsychology \& Behavior, 4(2), 183-201.

Schwind, V., Knierim, P., Hass, N., \& Henze, N. (2019). Using Presence Questionnaires in Virtual Reality. CHI Conference on Human Factors in Computing Systems Proceedings (CHI 2019). https://doi.org/10.1145/3290605.3300590

Sheridan, T. B. (1992). Musings on Telepresence and Virtual Presence. Presence: Teleoperators and Virtual Environments, 1(1), 120-126. https://doi.org/10.1162/pres.1992.1.1.120

Sivunen, A., \& Nordbäck, E. (2015). Social presence as a multi-dimensional group construct in 3D virtual environments. Journal of Computer-Mediated Communication, 20(1), 19-36. https://doi.org/10.1111/jcc4.12090

Skarbez, R., Brooks, Jr., F. P., \& Whitton, M. C. (2017). A Survey of Presence and Related Concepts. ACM Computing Surveys, 50(6), 1-39. https://doi.org/10.1145/3134301 
Slater, M., Usoh, M., \& Steed, A. (1994). Depth of presence in virtual environments1994Slater.pdf. Presence: Teleoperators and Virtual Environments, 3(2), $130-144$.

Slater, M., \& Wilbur, S. (1997). A framework for immersive virtual environments (FIVE): Speculations on the role of presence in virtual environments. Presence: Teleoperators and Virtual Environments, 6(6), 603-616. Retrieved from http://www.ncbi.nlm.nih.gov/pubmed/7851

Slater, M. (2009). Place illusion and plausibility can lead to realistic behaviour in immersive virtual environments. Philosophical Transactions of the Royal Society B: Biological Sciences, 364(1535), 3549-3557. https://doi.org/10.1098/rstb.2009.0138

Slater, M. (2018). Immersion and the illusion of presence in virtual reality. British Journal of Psychology, 109(3), 431-433. https://doi.org/10.1111/bjop.12305

Till, B. C., Masson, M. E. J., Bub, D. N., \& Driessen, P. F. (2014). Embodied Effects of Conceptual Knowledge Continuously Perturb the Hand in Flight. Psychological Science, 25(8), 1637-1648. https://doi.org/10.1177/0956797614538842

Triantafyllou, K. (2014). Virtual reality simulators for gastrointestinal endoscopy training. World Journal of Gastrointestinal Endoscopy, 6(1), 6. https://doi.org/10.4253/wjge.v6.i1.6

Weibel, D., \& Wissmath, B. (2011). Immersion in computer games: The role of spatial presence and flow. International Journal of Computer Games Technology, 2011. https://doi.org/10.1155/2011/282345

Wirth, W., Hartmann, T., Böcking, S., Vorderer, P., Klimmt, C., Schramm, H., ... Jäncke, P. (2007). A process model of the formation of spatial presence experiences. Media Psychology, 9(3), 493-525. https://doi.org/10.1080/15213260701283079

Witmer, B., \& Singer, M. (1998). Measuring presence in virtual environments: A presence questionnaire. Presence, 7(3), 225-240. https://doi.org/10.1117/12.2233447

Wong, A. L., \& Haith, A. M. (2017). Motor planning flexibly optimizes performance under uncertainty about task goals. Nature Communications, 8, 1-10. https://doi.org/10.1038/ncomms 14624

Zahorik, P., \& Jenison, R. L. (1998). Presence as being-in-the-world. Presence: Teleoperators and Virtual Environments, 7(1), 78-89. https://doi.org/10.1162/105474698565541 


\section{APPENDIX A: EXPERIMENT DOCUMENTS \\ SONA POSTING}

Study Name: Interacting with Objects in Virtual Reality

Experimenters: $\quad$ Robin Langerak, Ph.D. Student, Department of Psychology

(Undergraduate Volunteers)

Experimenter's Phone: 613-520-2600 ext. 2487

Experiment Location: VSIM Building, Room 2201

Description: In this experiment you will be presented with a virtual office environment through an Oculus Rift head-mounted display. Your task will be to reach for virtual target objects in the virtual environment When reaching for the targets, you may encounter additional objects in the virtual workspace. Some of these virtual objects may be aversive (e.g., smoke) while others may be positive or neutral (e.g., fog). You will also be asked to complete a short questionnaire about your experience in the virtual environment and the experimenter will ask you for any additional comments you may have about your experience. This research was approved by the Carleton University Research Ethics Board - B (Project Approval 15-186) on September 15, 2017.

Eligibility Requirements: Normal or corrected-to-normal visual acuity, right-handed, no motor control difficulties.

Duration: 1 hour

Remuneration: $1 \%$ course credit

Preparation: None

Exclusions: Please do not sign up for this study if you have motor control difficulties or are prone to feeling nauseous. 
INFORMED CONSENT FORM

Project Title: Interacting with Objects in Virtual Reality

Faculty Sponsor: Dr. Chris Herdman, Department of Psychology, Carleton University, tel. 520-2600 x. 8122 The purpose of this informed consent form is to ensure that you understand both the purpose of the study and the nature of your participation. The informed consent must provide you with enough information so that you have the opportunity to determine whether you wish to participate in the study. This research was approved by the Carleton University Research Ethics Board - B (Project Approval 15-186) on October 19, 2016. Please ask the researcher to clarify any concerns that you may have after reading this form.

Research Personnel: In addition to the Faculty Sponsor named above, the following people are involved in this research and may be contacted at any time should you require further information about this study:

\begin{tabular}{|l|l|l|l|l|}
\hline Name & Title & Department & Email & Phone \\
\hline Robin Langerak & Ph.D. Student & Psychology & robin_langerak@carleton.ca & $520-2600$ x. 2487 \\
\hline Kayla Pedret & $\begin{array}{l}\text { Undergraduate } \\
\text { Volunteer }\end{array}$ & Cognitive Science & kaylapedret@cmail.carleton.ca & $520-2600$ x. 2487 \\
\hline
\end{tabular}

Other Contacts: Should you have any ethical concerns regarding this study, please contact:

\begin{tabular}{|l|l|}
\hline Name & Contact Info. \\
\hline Carleton University Research Office & ethics@carleton.ca \\
\hline Dr. Shelley Brown, Chair, Carleton University Research Ethics Board - B & $520-2600$ x. 1505 \\
\hline
\end{tabular}

Purpose: The purpose of this study is to explore how people use vision to guide a virtual hand when making reaching motions toward objects in a virtual office environment.

Task: You will be asked to wear a head-mounted display (Oculus Rift) Once you are fitted with sensors and the head-mounted display you will be presented with a virtual office environment including a desk that you will appear to be sitting at. You will see a virtual representation of your arm as it is tracked. Your task will be to reach for and either touch or grasp virtual target objects that appear on the virtual desk. When reaching for the objects, you may encounter additional objects in the virtual workspace. Some of these virtual objects may be aversive (e.g., smoke) while others may be positive or neutral (e.g., fog). After this task has been completed, you will be asked to complete a short questionnaire and engage in a short conversation about your experience with the experimenter.

Duration, Locale \& Compensation: Testing will take place in VSIM 2201 and will take approximately one and a half hours. You will receive $1.5 \%$ course credit for your participation.

Potential Risks/Discomfort: Some participants may feel a bit nauseous. Please alert the experimenter if you need a break or wish to stop.

Anonymity/Confidentiality: All data collected in this experiment will be kept strictly confidential and will be anonymized through the assignment of a coded number and securely stored on a local server for a maximum of ten years. Aggregated motion data will be kept in cloud storage behind 2-factor authentication protection and retained for a maximum of ten years. This Informed Consent form will be kept for a maximum of ten years before being destroyed. The information provided will be used for research purposes only. You will not be identified by name in any reports produced from this study. Further, the information is made available only to the researchers associated with this experiment

Right to Withdraw/Omit: You have the right to withdraw from this experiment at any time without academic penalty. Your participation in this experiment is completely voluntary, please alert the experimenter if you wish to withdraw. 
I have read the above description of the study on interacting with virtual objects. By signing below, this indicates that I agree to participate in the study, and this in no way constitutes a waiver of my rights.

Name:

Signature:
Date:

Witness: 


\section{Virtual Reality Experience Questionnaire}

Please rate your agreement with the following statements by placing a circle on the line according to the scale provided.

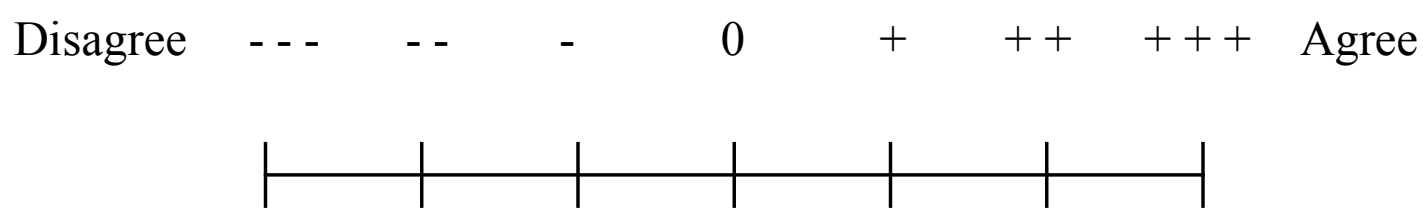

1. It seemed as if I were seeing the virtual hand/cube in the location where I felt my hand to be.

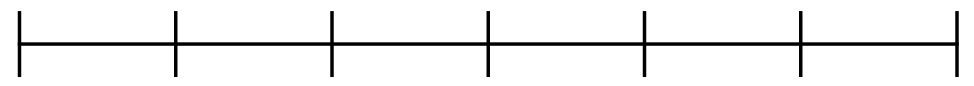

2. It seemed as though the virtual hand/cube hitting the target was caused by the motion of my own hand.

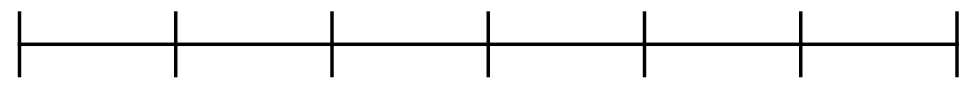

3. Even though the virtual hand/cube I saw did not look like mine, I felt as if it was my own.

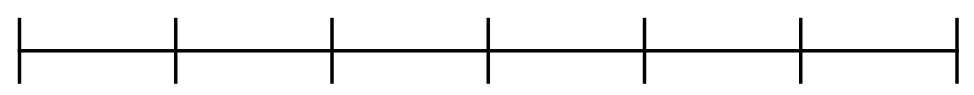

4. It seemed as if I might have more than one hand.

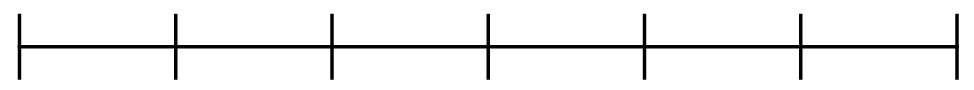

5. I felt that the virtual hand/cube that I saw belonged to someone else.

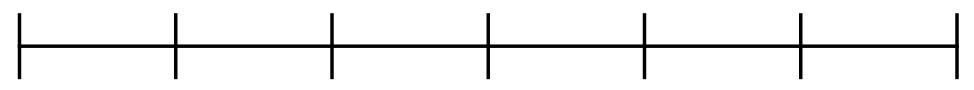

6. It felt like something was off about the movement of the hand/cube for an unknown reason.

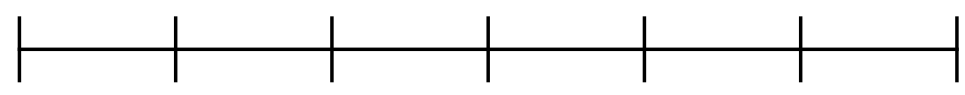




\section{DEBRIEFING FORM}

\section{Interacting with Objects in Virtual Reality}

Thank you for participating in this experiment exploring how people interact with virtual objects. We plan to examine whether there are differences in how people make reaching motions in virtual environments as compared to real environments. We are also interested in determining if people feel a sense of ownership over virtual representations of their hands by observing what happens when they reach for targets in the presence of "aversive" stimuli - such as smoke - as compared to positive or neutral stimuli - such as fog. From this we hope to learn more about how the brain uniquely represents the space near our hands and how vision interacts with the presence of our hands, in both real and virtual environments.

If you are interested in learning more about this study or the topic, please see the following:

Botvinick, M. \& Cohen, J. (1998). Rubber hands 'feel' touch than eyes see. Nature, 391: 756.

Fuentes, C. \& Bastian, A. (2010). Where is your arm? Variations in proprioception across space and tasks. Journal of Neurophysiology, 103: 164-171.

This research was approved by the Carleton University Research Ethics Board - B (Project Approval 15-186) on October 19, 2015. Should you have any ethical concerns regarding this study then please contact:

\begin{tabular}{|c|c|}
\hline Name & Contact Info. \\
\hline Carleton University Research Office & ethics@carleton.ca \\
\hline Dr. Shelly Brown, Chair, Carleton University Research Ethics Board - B & $520-2600$ x. 1505 \\
\hline
\end{tabular}

Should you have any other concerns about this study then please contact any of the following individuals:

\begin{tabular}{|l|l|l|l|l|}
\hline Name & Title & Department & Study Role & Contact Info. \\
\hline Robin Langerak & Ph.D. Student & Psychology & Principal Investigator & robin.langerak@carleton.ca \\
\hline Kayla Pedret & $\begin{array}{l}\text { Undergraduate } \\
\text { Volunteer }\end{array}$ & Cog. Science & Co-Investigator & kaylapedret@cmail.carleton.ca \\
\hline Dr. Chris Herdman & Professor & Psychology & Faculty Supervisor & chris_herdman@carleton.ca \\
\hline $\begin{array}{l}\text { Dr. Anthony } \\
\text { Whitehead }\end{array}$ & Professor & $\begin{array}{l}\text { Human- } \\
\text { Computer } \\
\text { Interaction }\end{array}$ & Faculty Co-Supervisor & anthony.whitehead@carleton.ca \\
\hline
\end{tabular}




\section{APPENDIX B: DATA PREPARATION \\ DATA TRIMMING}

There were a number of trials upon which participants made anticipatory movements before the target appeared. These trials were automatically coded by the experiment program as mistrials and were discarded from the analysis, resulting in $7 \%$ data loss. Further inspection of the data revealed the occurrence of two different types of mistrials that were not captured by the experiment program. These mistrials were discovered when the reported final depth position of the motion controller was mere centimeters from the start position; this is demonstrated in the bimodal distribution of controller end positions in Appendix B: Data preparation. When these data were scrutinized together with their associated motion time it was revealed that two different types of mistrials were occurring. The first type of mistrial was that participants' stops at the target were not captured. The manner in which motion initiation and termination were detected depended on the thresholds set within the experiment program. It was therefore possible for a participant to stop at targets too abruptly for their motion to drop below the set threshold for the program to capture their stop at the target. On these trials the motion controller's stop at the target went undetected and the program did not report the controller stopping until it was back at the start position. The second type of mistrial was when participants' velocity dropped below the stop threshold before they had reached the target. Accordingly, all trials with a stop location within $10 \mathrm{~cm}$ of the start position were discarded as mistrials, resulting in a new total data loss of $16 \%$.

For the remaining data there were still some cases where the final position of the motion controller at a target was not detected. Thus, all trials on which travel time was greater than $1000 \mathrm{~ms}$ were discarded. Similarly, those trials where liftoff time was greater than $1000 \mathrm{~ms}$ were 


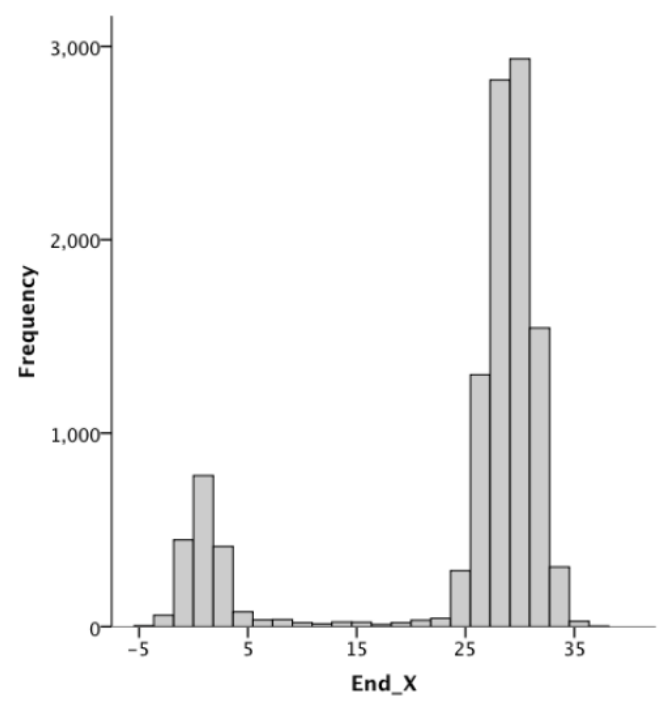

Figure 22. Histogram showing distribution of recorded final depth positions in $\mathrm{cm}$ (End_X) of the motion controller, negative numbers represent positions behind the start position and positive numbers represent final positions between the start position and targets, where targets are arranged at 27, 29, and $30 \mathrm{~cm}$ away from start.

removed under the assumption that these trials reflected a case in which the participant was not prepared to reach quickly or were moving too slowly for the program to capture their true motion start. There were also some anomalous cases where the liftoff time as well as the total travel times of the motion controller were well below reasonable. These cases were considered as a mistrial that was not automatically caught by the program and all trials where travel times or liftoff time were below 200ms were discarded. These travel times and liftoff time box trimming procedures resulted in a further $1 \%$ data loss. Finally, both liftoff time and travel times were subjected to an outlier trimming procedure based upon z-scores calculated separately by participant and by reaching hand. Those liftoff time and travel times values outside of 3SD of their respective cells were removed from the dataset, resulting in a further $3 \%$ data loss for a total of $20 \%$ data loss due to a combination of program threshold error and latency trimming procedures. 


\section{CALCULATIONS OF EUCLIDEAN DISTANCE AND ACCURACY}

Sample calculations for Experiment 1

Let $c$ represent the distance of the effector from the target where the two objects are adjacent without overlapping. To ensure that accuracy coding results in a 0 code for all trials where it is absolutely certain there was no overlap, the line from the centre of the effector (where the experiment program records its location) to a corner will be used as this is the farthest point from the effector's centre and thus will serve as the closest possible distance measurement that satisfies the zero overlap criterion. The length of $\mathrm{x}$ was found using known dimensions of the effector and the angle-side-angle rule to solving a triangle.

$$
\begin{aligned}
\text { side-angle-side }=\frac{b}{\sin B} & =\frac{c}{\sin C} \\
\frac{5}{\sin 90} & =\frac{c}{\sin 45} \\
\frac{5}{1} & =\frac{c}{0.7150} \\
3.54 & =c
\end{aligned}
$$

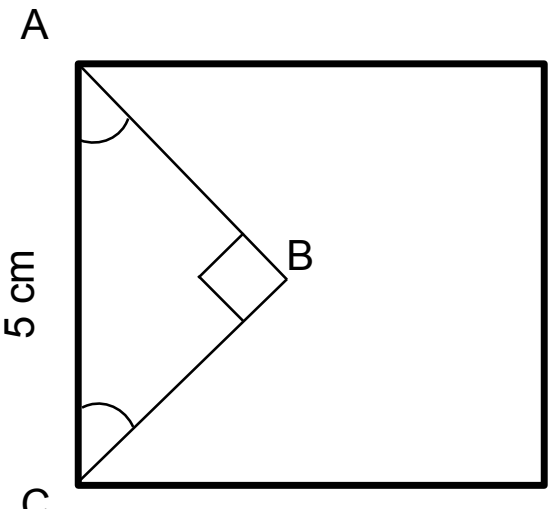

Effector

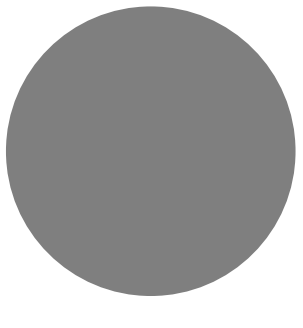

Target

Given that the radius of the target is $1.5 \mathrm{~cm}$, let $l$ be total length,

$l=1.5+3.54$

$l=5.04$

Therefore, the total length from target centre to effector centre, when the objects are adjacent without overlapping is $5.04 \mathrm{~cm}$. Thus, landing accuracy will be coded as:

Distance between effector and target $<5.04=1$ (on target)

Distance between effector and target $>5.04=0$ (missed) 


\section{APPENDIX E: TECHNICAL SPECIFICATIONS}

\section{ALTERATIONS TO LEAP MOTION TRACKING PROTOCOL}

The Leap Motion controller ran version 3.13 of the Orion software which was built specifically to enable hand tracking for VR applications. Some alterations were made to the native Leap Motion tracking such that the tracking origin of the Leap Motion controller was calibrated to match its physical position on the Oculus Rift headset rather than a fixed location mounted somewhere in the workspace. This relocation of the tracking origin was performed to correct for any tilt or roll head motion introduced by mounting the Leap Motion controller onto the Oculus Rift headset, thus ensuring that the virtual location of participants' hands matched their physical location.

In addition to this re-calibration of the tracking origin some alterations were made to the tracking protocol to account for positional shifts of participants' heads. It was discovered that when the headset wearer was making a reach, involuntary head movement caused inaccuracies in the physical-to-virtual hand tracking resulting in an unstable hand visualization. This instability was caused by an approximately 50 millisecond delay between capture of participants' physical hand location and display of the virtual representation of their hands. The nature of this instability was such that the virtual hands' location was coerced toward inaccurate positions by small movements of the head; while the Leap Motion controller was detecting the true position of the hands it used information about head movement to move the most recently updated hand position to the newly moved to visual reference point. One example of this unstable hand tracking presented as a kind of telescoping of the arm. When the head and torso, and therefore the Leap Motion controller, moved forward with a reach the virtual hands were pushed forward to appear further away from the body than they were. To compensate for this delay the inverse of 
any detected head motion was applied to the virtual tracking origin of the Leap Motion controller over a 50 millisecond window. This resulted in a more stable tracking and visualization of the hands.

In addition to this altered tracking protocol a custom anti-aliasing algorithm was written to adjust for the differences between the Leap Motion controller's tracking framerate $(115 \mathrm{~Hz})$ and the Oculus Rift's visual refresh framerate $(90 \mathrm{~Hz})$. Due to the differences in tracking and display refresh rates, or temporal aliasing, it was discovered that participants would observe substantial visual disruptions and visual artifacts, or judder, in the virtual representation of their hand movements. To correct for this the algorithm applied an anti-aliasing function whereby late, missing, or temporally mismatched frame updates from the Leap Motion to the Oculus Rift were substituted with the most recent frame from a temporarily stored buffer of hand position frames. This anti-aliasing function allowed for better synchrony between the framerates of these two pieces of equipment resulting in a more accurate representation of participants' hand movements. These alterations to the Leap Motion controller's tracking protocol were applied to both the virtual hand and virtual cube representations described in the Display and Stimuli section.

The final idiosyncrasy of the Leap Motion controller worth noting is that it detects participants hands by matching visual information from the environment to a visual comparator of certain shape and size. An artifact of this visual comparison process was that when participants' hands were not the same size as this comparator hand they were deemed by the Leap Motion as being slightly closer (large hands) or further (small hands) from the body than they truly were. This size comparison artifact resulted in small discrepancies between the physical and virtual location of hands placed directly on the table. A work around was implemented where the visual height of the virtual table was adjusted for each participant so that 
their virtual hand - or their virtual cube - always appeared above the table at the start and target positions. This calibration process is described in the Procedure section of Experiment 2. 